\title{
Equivalences of tame blocks for $p$-adic linear groups
}

\author{
Jean-François Dat*
}

March 2016

\begin{abstract}
Let $p$ and $\ell$ be two distinct primes, $F$ a $p$-adic field and $n$ an integer. We show that any

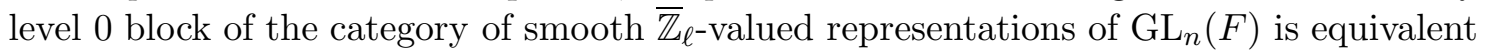
to the unipotent block of an appropriate product of $\mathrm{GL}_{n_{i}}\left(F_{i}\right)$. To this end, we first show that the level 0 category of $\mathrm{GL}_{n}(F)$ is equivalent to a category of "modules" over a certain

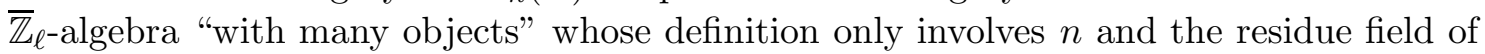
$F$. Then we use fine properties of Deligne-Lusztig cohomology to split this algebra and produce suitable Morita equivalences.
\end{abstract}

\section{Contents}

1 Introduction $\quad 2$

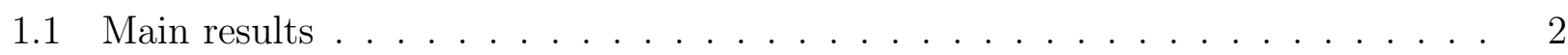

1.2 Outline of the construction ...................... 4

2 Finite linear groups

2.1 Review of Lusztig theory . . . . . . . . . . . . . . . . . . . . . . . . . .

2.2 General linear groups in a coordinate-free setting . . . . . . . . . . . 11

3 From level 0 blocks to coefficient systems

3.1 Review of coefficients systems and systems of idempotents . . . . . . . . 16

3.2 Level 0 blocks . . . . . . . . . . . . . . . . . . . . . . . 18

4 From Coefficient systems to Hecke "modules" 22

4.1 The enriched Coxeter complex . . . . . . . . . . . . . . 22

4.2 Hecke "algebras" and "modules" . . . . . . . . . . . . . . . 25

4.3 Morita equivalence of Hecke "algebras" . . . . . . . . . . . . . . . . 27

A "Rings" and "modules" 34

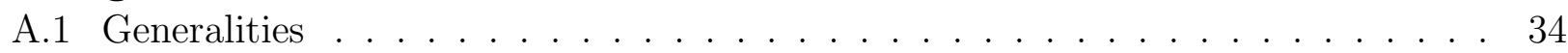

A.2 Morita equivalences . . . . . . . . . . . . . . . . . . 38

*The author thanks the Institut Universitaire de France and the ANR project ANR-14-CE25-0002 PerCoLaTor for their support. 


\section{Introduction}

Let $F$ be a $p$-adic field and let $R$ be a commutative ring in which $p$ is invertible. For $\mathbf{G}$ a reductive group over $F$, we put $G:=\mathbf{G}(F)$ and we denote by $\operatorname{Rep}_{R}(G)$ the abelian category of smooth representations of $G$ with coefficients in $R$, and by $\operatorname{Irr}_{R}(G)$ the set of isomorphism classes of simple objects in $\operatorname{Rep}_{R}(G)$. In this paper we focus on the following situation :

- $\mathbf{G}$ is of GL-type, by which we mean that $\mathbf{G}$ is a product of restrictions of scalars of general linear groups.

- $R=\overline{\mathbb{Z}}_{\ell}$, for $\ell$ a prime number coprime to $p$, occasionnally $R=\overline{\mathbb{F}}_{\ell}$ or $\overline{\mathbb{Q}}_{\ell}$.

\subsection{Main results}

1.1.1 The block decomposition. - For $\mathbf{G}=\mathrm{GL}_{n}$, Vignéras [9] has obtained a decomposition of the category $\operatorname{Rep}_{\overline{\mathbb{F}}_{\ell}}(G)$ as a product of blocks, indexed by the so-called "inertial classes of supercuspidal pairs" $[M, \pi]$ with $M$ a Levi subgroup and $\pi \in \operatorname{Irr}_{\bar{F}_{\ell}}(M)$ supercuspidal. This decomposition was further lifted to a decomposition of $\operatorname{Rep}_{\overline{\mathbb{Z}}_{\ell}}(G)$ by Helm in [6]. A simple object of $\operatorname{Rep}_{\overline{\mathbb{Z}}_{\ell}}(G)$ belongs to $\operatorname{Rep}_{[M, \pi]}(G)$ if and only if it has "mod $\ell$ inertial supercuspidal support" equal to $[M, \pi]$, in the sense of [6, Def. 4.10].

At the moment, Vigneras blocks and Helm blocks are not well understood. Actually even the structure of the principal block of $\operatorname{Rep}_{\overline{\mathbb{Z}}_{\ell}}(G)$ (the one that contains the trivial representation, and corresponds to the supercuspidal pair $[T, 1]$ with $T$ a maximal torus) is still a mystery.

However, it is expected that any Vigneras or Helm block is equivalent to the principal block of another group $\mathbf{G}^{\prime}$ of GL-type. One consequence of this paper is the following result in this direction.

Theorem. - If $\mathbf{G}$ is of GL-type, any level 0 block of $\operatorname{Rep}_{\overline{\mathbb{Z}}_{\ell}}(G)$ is equivalent to the principal block of $\operatorname{Rep}_{\overline{\mathbb{Z}}_{\ell}}\left(G^{\prime}\right)$ for an appropriate $\mathbf{G}^{\prime}$ of $\mathrm{GL}$-type.

Here, a block of $\operatorname{Rep}_{\overline{\mathbb{Z}}_{\ell}}(G)$ has level 0 if any object in it has non-zero invariants under the first congruence subgroup of some parahoric group in $G$. In terms of supercuspidal pair $[M, \pi]$, this means that $\pi$ has level 0 (or "depth 0 ") in the usual sense.

In this result, the group $\mathbf{G}^{\prime}$ may not be unique. This is already the case for $\overline{\mathbb{Q}}_{\ell}$-coefficients since the Iwahori-Hecke algebra of $\mathrm{GL}_{n}(F)$ only depends on the residual field of $F$. However, we have explained in [5] how a very natural $\mathbf{G}^{\prime}$ stands out when we take the Langlands-dual point of view.

1.1.2 Dual parametrization of blocks. - Let $W_{F}$ denote the absolute Weil group of $F$, and let $I_{F}$ be its inertia subgroup. We will denote by $I_{F}^{\ell}$ the prime-to- $\ell$ radical of $I_{F}$, i.e. the kernel of the canonical map $I_{F} \longrightarrow \mathbb{Z}_{\ell}(1)$. We have observed in [5, §1.2.1] that Vignéras' Langlands correspondence for supercuspidal $\overline{\mathbb{F}}_{\ell^{-}}$-representations of $\mathrm{GL}_{n}(F)$ yields a bijection $[M, \pi] \leftrightarrow \phi$ between inertial classes of supercuspidal pairs and isomorphism classes of semi-simple continous representations $I_{F}^{\ell} \longrightarrow \mathrm{GL}_{n}\left(\overline{\mathbb{Q}}_{\ell}\right)$ that can be extended to $W_{F}$.

More generally, let ${ }^{L} \mathbf{G}=\hat{\mathbf{G}} \rtimes W_{F}$ denote "the" $L$-group of a connected reductive group over $F$ and let $\Phi\left(\mathbf{G}, \overline{\mathbb{Q}}_{\ell}\right) \subset H^{1}\left(W_{F}^{\prime}, \hat{\mathbf{G}}\left(\overline{\mathbb{Q}}_{\ell}\right)\right)$ denote the set of admissible Weil-Deligne parameters 
for $\mathbf{G}$. When $\mathbf{G}$ is of GL-type, we again refer to [5, §1.2.1] for a bijection $[M, \pi] \leftrightarrow \phi$ where now $\phi$ belongs to the set

$$
\Phi_{\ell-\text { inert }}\left(\mathbf{G}, \overline{\mathbb{Q}}_{\ell}\right)=\operatorname{im}\left(\Phi\left(\mathbf{G}, \overline{\mathbb{Q}}_{\ell}\right) \stackrel{\text { rest }}{\longrightarrow} H^{1}\left(I_{F}^{\ell}, \hat{\mathbf{G}}\left(\overline{\mathbb{Q}}_{\ell}\right)\right)\right) .
$$

Therefore, to any $\phi \in \Phi_{\ell \text {-inert }}\left(\mathbf{G}, \overline{\mathbb{Q}}_{\ell}\right)$ is attached a block $\operatorname{Rep}_{\phi}(G)$ of $\operatorname{Rep}_{\overline{\mathbb{Z}}_{\ell}}(G)$. The irreducible $\overline{\mathbb{Q}}_{\ell}$-representations $\pi$ in this block are those whose Langlands parameter $\varphi_{\pi}$ satisfies $\left(\varphi_{\pi}\right)_{\mid I_{F}^{\ell}} \sim \phi$. In this parametrization, the principal block corresponds to the trivial parameter $\phi: i \mapsto(1, i)$, while level 0 blocks correspond to tame parameters $\phi$, in the sense that the restriction $\phi_{\mid P_{F}}$ to the wild inertia subgroup $P_{F}$ is trivial.

1.1.3 The transfer conjecture. - Having a dual parametrization suggests that some kind of "Langlands transfer" principle for blocks might exist. So, let us consider a $L$-homomorphism $\xi:{ }^{L} \mathbf{G}^{\prime} \longrightarrow{ }^{L} \mathbf{G}$ of groups of GL-type, fix an admissible parameter $\phi^{\prime}: I_{F}^{(\ell)} \longrightarrow{ }^{L} \mathbf{G}^{\prime}$ and put $\phi:=\xi \circ \phi^{\prime}$. Let us denote by $C_{\hat{\mathbf{G}}}(\phi)$ the centralizer of the image of $\phi$. We have formulated the following conjecture in [5, $\S 1.2 .2]$

Conjecture. - Suppose that $\xi$ induces an isomorphism $C_{\hat{\mathrm{G}}^{\prime}}\left(\phi^{\prime}\right) \stackrel{\sim}{\longrightarrow} C_{\hat{\mathbf{G}}}(\phi)$, and also that the projection of $\xi\left(W_{F}\right)$ to $\hat{G}\left(\overline{\mathbb{Q}}_{\ell}\right)$ is bounded. Then there is an equivalence of categories $\operatorname{Rep}_{\phi^{\prime}}\left(G^{\prime}\right) \stackrel{\sim}{\longrightarrow} \operatorname{Rep}_{\phi}(G)$ that interpolates the Langlands transfer $\xi_{*}$ on irreducible $\overline{\mathbb{Q}}_{\ell^{-}}$ representations.

1.1.4 Main results. - In this paper we construct an equivalence $\operatorname{Rep}_{\phi^{\prime}}\left(G^{\prime}\right) \stackrel{\sim}{\longrightarrow} \operatorname{Rep}_{\phi}(G)$ in the following two cases.

i) $\phi^{\prime}=1$ and $\xi$ is a base change $L$-morphism ${ }^{L} \mathrm{GL}_{n} \longrightarrow{ }^{L} \operatorname{Res}_{F^{\prime} \mid F}\left(\mathrm{GL}_{n}\right)$ with $F^{\prime}$ totally ramified.

ii) $\phi^{\prime}$ is tame and $\xi$ is an automorphic induction $L$-morphism ${ }^{L} \operatorname{Res}_{F^{\prime} \mid F}\left(\mathrm{GL}_{n^{\prime}}\right) \longrightarrow{ }^{L} \mathrm{GL}_{n}$, where $F^{\prime}$ is unramified and $n=n^{\prime}\left[F: F^{\prime}\right]$.

We have explained in [5, §2.4] how these two particular cases imply the following weak form of the above conjecture for tame parameters and tame $L$-morphisms.

Theorem. - Let $\xi$ be as in the conjecture, and suppose that both $\xi$ and $\phi^{\prime}$ are tam 1 . Then there is an equivalence of categories $\operatorname{Rep}_{\phi^{\prime}}\left(G^{\prime}\right) \stackrel{\sim}{\longrightarrow} \operatorname{Rep}_{\phi}(G)$.

This result, in turn, implies the following more precise version of Theorem 1.1.1, for which we refer again to $[5, \S 1.2 .3]$. For any $\phi \in \Phi_{\ell-\text { inert }}\left(\mathbf{G}, \overline{\mathbb{Q}}_{\ell}\right)$ there is a unique connected reductive group $\mathbf{G}_{\phi}$ of GL-type over $F$, such that $\hat{\mathbf{G}}_{\phi}=C_{\hat{\mathbf{G}}}(\phi)$ and ${ }^{L} \mathbf{G}_{\phi}$ is isomorphic to the normalizer $\mathcal{N}_{L} \mathbf{G}(\phi)$ of the image of $\phi$ in ${ }^{L} \mathbf{G}$. Moreover $\mathbf{G}_{\phi}$ is unramified if $\phi$ is tame.

Corollary. - For any tame parameter $\phi$ of a group $\mathbf{G}$ of GL-type, there is an equivalence of categories $\operatorname{Rep}_{1}\left(G_{\phi}\right) \stackrel{\sim}{\longrightarrow} \operatorname{Rep}_{\phi}(G)$.

What the theorem and the corollary are missing at the moment is the compatibility with the transfer maps on irreducible $\overline{\mathbb{Q}}_{\ell}$-representations. This would follow from a natural and hoped-for compatibility with parabolic induction of our explicit constructions in cases i) and ii) above, that we haven't proved yet.

\footnotetext{
${ }^{1}$ this means that the restrictions of $\xi$ and $\phi^{\prime}$ to $P_{F}$ are $\hat{\mathbf{G}}$-conjugate to the map $\gamma \mapsto(1, \gamma)$
} 


\subsection{Outline of the construction}

Here we outline the construction of the desired equivalences in the two settings i) and ii) of

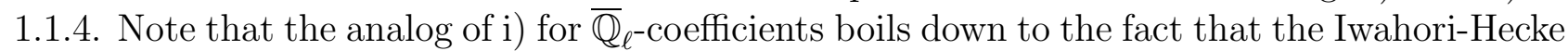

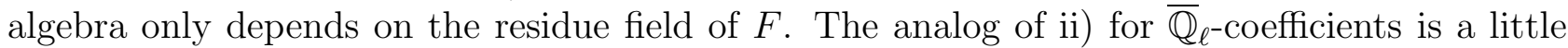
more complicated and uses the computation of Hecke algebras for level 0 simple types (in the

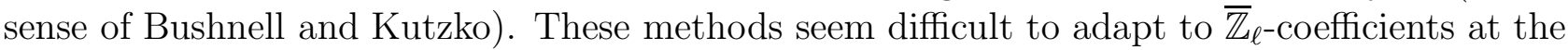
moment, because there is no known connection between blocks and categories of modules over sufficiently explicit algebras. Our method is therefore completely different. It relies on modular Deligne-Lusztig theory, that provides a crucial input from representation theory of finite linear groups, and on coefficient systems on the building, that provide the necessary link between finite and $p$-adic linear groups. Technically, we replace "Hecke algebras" by "Hecke algebras with many objects", see 1.2.4.

1.2.1 Tame inertial parameters of $\mathrm{GL}_{n}(F)$ and semisimple conjugacy classes in $\mathrm{GL}_{n}(\mathbb{F})$. Recall that the quotient $I_{F} / P_{F}$ is procyclic of pro-order prime to $p$. More precisely, let us fix an isomorphism $\iota:(\mathbb{Q} / \mathbb{Z})_{p^{\prime}} \stackrel{\sim}{\longrightarrow} \overline{\mathbb{F}}^{\times}$, where $\mathbb{F}=\mathbb{F}_{q}$ is the residue field of $F$. This lifts uniquely to an embedding $\iota:(\mathbb{Q} / \mathbb{Z})_{p^{\prime}} \hookrightarrow \bar{F}^{\times}$, and provides us with a generator of $I_{F} / P_{F}=\mathbb{Z}_{p^{\prime}}(1)$, namely the system $\gamma:=\left(\iota\left(\frac{1}{N}\right)\right)_{(N, p)=1}$. Therefore, a continuous morphism $\phi: I_{F} / P_{F} \longrightarrow \mathrm{GL}_{n}\left(\overline{\mathbb{Q}}_{\ell}\right)$ (with discrete topology on target) is given by the image $s=\phi(\gamma)$, which has finite and primeto- $p$ order. Such a morphism extends to $W_{F}$ if and only if $s^{q}$ is conjugate to $s$. Hence a tame parameter $\phi \in \Phi_{\text {inert }}\left(\mathrm{GL}_{n}, \overline{\mathbb{Q}}_{\ell}\right)$ is given by a conjugacy class in $\mathrm{GL}_{n}\left(\overline{\mathbb{Q}}_{\ell}\right)$ stable under $q^{t h}$-power. Such a conjugacy class is determined by its characteristic polynomial $P_{\phi}(X) \in \overline{\mathbb{Q}}_{\ell}[X]$, which has the form $P_{\phi}(X)=\prod_{i=1}^{n}\left(X-\zeta_{i}\right)=\prod_{i=1}^{n}\left(X-\zeta_{i}^{q}\right)$ with $\zeta_{i}$ a $p^{\prime}$-root of unity.

Let us now choose an embedding $\jmath:(\mathbb{Q} / \mathbb{Z})_{p^{\prime}} \hookrightarrow \overline{\mathbb{Z}}_{\ell}^{\times}$. Via $\iota \circ \jmath^{-1}$ we can transfer $P_{\phi}(X)$ to a polynomial in $\overline{\mathbb{F}}[X]$, and the functional equation of $P_{\phi}(X)$ says that this polynomial is in fact in $\mathbb{F}[X]$. Therefore it corresponds to a semisimple conjugacy class $s$ in $\mathrm{GL}_{n}(\mathbb{F})$.

In the same way, a tame $\ell$-inertia parameter $I_{F}^{\ell} \longrightarrow \mathrm{GL}_{n}\left(\overline{\mathbb{Q}}_{\ell}\right)$ corresponds to a semisimple conjugacy class $s$ in $\mathrm{GL}_{n}(\mathbb{F})$ whose elements have prime-to- $\ell$ order. This fits into a diagram

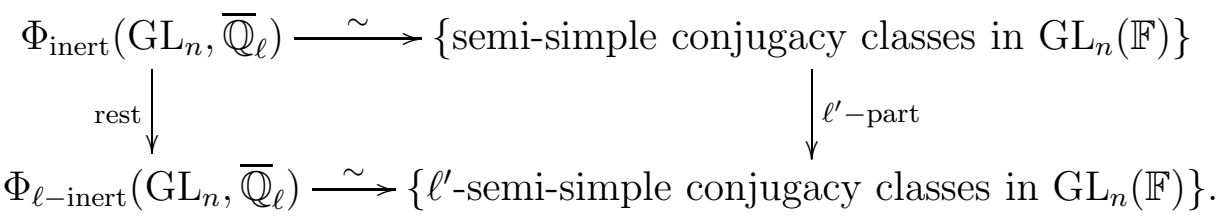

1.2.2 Semisimple conjugacy classes and idempotents in $\overline{\mathbb{Z}}_{\ell} \mathrm{GL}_{n}(\mathbb{F})$. - Green's classification of irreducible representations of the finite group $\mathrm{GL}_{n}(\mathbb{F})$ provides us with a surjective map $\operatorname{Irr}_{\overline{\mathbb{Q}}_{\ell}}\left(\mathrm{GL}_{n}(\mathbb{F})\right) \longrightarrow \mathrm{GL}_{n}(\mathbb{F})_{\text {/conj }}^{\mathrm{ss}}$, which a priori depends on our choices of $\iota$ and $\jmath$. This map can also be understood in terms of Deligne-Lusztig "twisted" induction, and its fibers are called "(Lusztig) rational series" (see section 2 for a brief account). Let $s$ be a semisimple conjugacy class in $\mathrm{GL}_{n}(\mathbb{F})$ of order prime to $\ell$. Broué and Michel have proved that the central idempotent

$$
e_{s}:=\sum_{\left(s^{\prime}\right)_{\ell^{\prime}}=s} \sum_{\pi \mapsto s^{\prime}} e_{\pi}
$$


of $\overline{\mathbb{Q}}_{\ell} \mathrm{GL}_{n}(\mathbb{F})$ actually lies in $\overline{\mathbb{Z}}_{\ell} \mathrm{GL}_{n}(\mathbb{F})$. Here $e_{\pi} \in \overline{\mathbb{Q}}_{\ell} \mathrm{GL}_{n}(\mathbb{F})$ is the central idempotent attached to the irreducible representation $\pi$.

1.2.3 $\operatorname{Rep}_{\phi}(G)$ as a category of coefficient systems. - Denote by BT the reduced building of $\mathrm{GL}_{n}(F)$ with its simplicial structure. A vertex $x$ of $B T$ corresponds to an homothety class of $\mathcal{O}_{F}$-lattices, so its compact stabilizer comes with a reduction map $G_{x}^{0} \longrightarrow \mathrm{GL}_{n}(\mathbb{F})$ well defined up to inner automorphism of the target. Denote by $s$ the conjugacy class in $\mathrm{GL}_{n}(\mathbb{F})$ that corresponds to $\phi$ as in 1.2 .1 .

Taking inverse image of $e_{s}$ by the reduction map, we get a central idempotent $e_{x}^{\phi}$ in the smooth distribution algebra $\mathcal{H}_{\overline{\mathbb{Z}}_{\ell}}\left(G_{x}^{0}\right)$. More generally, the compact fixator $G_{\sigma}^{0}$ of a facet comes with a reduction map to a standard Levi subgroup of $\mathrm{GL}_{n}(\mathbb{F})$, and we can again define a central idempotent $e_{\sigma}^{\phi}$ in $\mathcal{H}_{\overline{\mathbb{Z}}_{\ell}}\left(G_{\sigma}^{0}\right)$.

Consider the category $\operatorname{Coef}_{e^{\phi}}^{G}(B T)$ of all $G$-equivariant coefficient systems in $\overline{\mathbb{Z}}_{\ell \text {-modules }}$ $\left(\mathcal{V}_{\sigma}\right)_{\sigma \in B T}$. on $B T$ such that for all $\tau \leqslant \sigma$, the structural map $\mathcal{V}_{\sigma} \longrightarrow \mathcal{V}_{\tau}$ is an isomorphism $\mathcal{V}_{\sigma} \stackrel{\sim}{\longrightarrow} e_{\sigma}^{\phi} \mathcal{V}_{\tau}$. Using results of Meyer-Solleveld [8] and Wang [10] we prove in part [3] that the functor which takes $V \in \operatorname{Rep}_{\overline{\mathbb{Z}}_{\ell}}(G)$ to the $G$-equivariant coefficient system $\left(e_{\sigma}^{\phi} V\right)_{\sigma \in B T}$. induces an equivalence of categories

$$
\operatorname{Rep}_{\phi}(G) \stackrel{\sim}{\longrightarrow} \operatorname{Coef}_{e^{\phi}}^{G}(B T) .
$$

1.2.4 $\operatorname{Rep}_{\phi}(G)$ as a category of modules over a Hecke "algebra". - In 4.1 .2 we introduce a category $[\mathbb{A} / \overline{\mathbb{G}}]$, that only depends on $n$ and on the residual field $\mathbb{F}$ of $F$. We start with the category $[\mathbb{A} / W]$ given by the Coxeter complex attached to the affine Weyl group $W$ of type $\widetilde{A}_{n-1}$ with its natural action by $W$. Then we enrich it by plugging in, for each simplex $\sigma$, a group of automorphisms $\overline{\mathbb{G}}_{\sigma}$ isomorphic to a well-chosen product of finite $\mathrm{GL}_{n_{i}}(\mathbb{F})$. Finally we

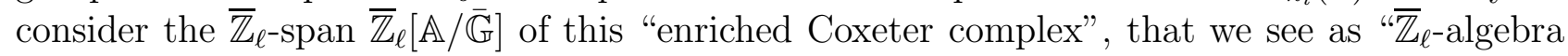
with many objects". The appendix recollects some supposedly standard definitions regarding the notion of modules on such gadgets.

To any semisimple conjugacy class $s$ in $\mathrm{GL}_{n}(\mathbb{F})$, we also attach an "algebra with many objects" $e_{s} \overline{\mathbb{Z}}_{\ell}[\mathbb{A} / \overline{\mathbb{G}}]$, and we show in 4.2 .2 that $\operatorname{Rep}_{\phi}(G)$ is naturally equivalent to the category of "cartesian modules" on this "algebra" for $s=s(\phi)$. The proof of this result takes the previous one about coefficient systems on the building as a starting point.

The fact that the construction of $e_{s} \overline{\mathbb{Z}}_{\ell}[\mathbb{A} / \overline{\mathbb{G}}]$ only depends on $n, \mathbb{F}$ and $s$ shows that we have in particular constructed an equivalence of categories as claimed in 1.1.4 i).

1.2.5 Automorphic induction setting. - Now, let $F^{\prime}, n^{\prime}$ and $\xi$ be as in 1.1.4 ii), and let $\mathbb{F}^{\prime}$ be the residual field of $\mathbb{F}$, which is an extension of degree $n^{\prime}$ of $\mathbb{F}$. In order to simplify the notation a bit, put $\mathbf{G}:=\mathrm{GL}_{n}$ and $\mathbf{G}^{\prime}:=\operatorname{Res}_{F^{\prime} \mid F}\left(\mathrm{GL}_{n^{\prime}}\right)$. We have a commutative diagram

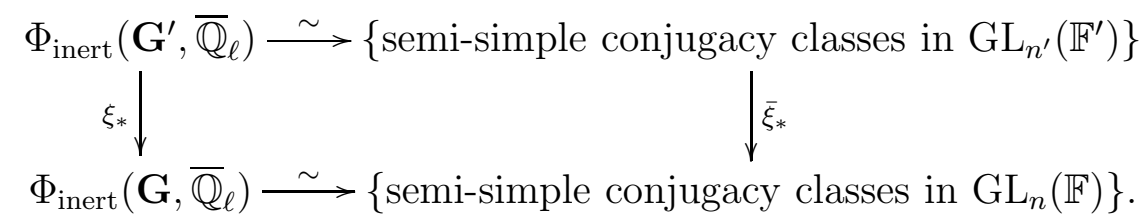

Here, the second line is the bijection explained in 1.2.1, while the first line is the same bijection for $\mathrm{GL}_{n^{\prime}, F^{\prime}}$, composed with the Shapiro bijection $\Phi_{\text {inert }}\left(\mathbf{G}^{\prime}, \overline{\mathbb{Q}}_{\ell}\right) \leftrightarrow \Phi_{\text {inert }}\left(\mathrm{GL}_{n^{\prime}, F^{\prime}}, \overline{\mathbb{Q}}_{\ell}\right)$ of $[5$, 
Corollary 2.3.3]. The left vertical map is composition with $\xi$, and the right vertical one $\bar{\xi}_{*}$ is the transfer of conjugacy classes that one gets through any embedding $\mathrm{GL}_{n^{\prime}}\left(\mathbb{F}^{\prime}\right) \hookrightarrow \mathrm{GL}_{n}(\mathbb{F})$ obtained by choosing an $\mathbb{F}$-basis of $\mathbb{F}^{\prime n^{\prime}}$. In this setup, the hypothesis that $\xi$ induces an isomorphism $C_{\hat{\mathbf{G}}^{\prime}}\left(\phi^{\prime}\right) \stackrel{\sim}{\longrightarrow} C_{\hat{\mathbf{G}}}(\phi)$ translates into the hypothesis that the centralizer in $\mathrm{GL}_{n}(\mathbb{F})$ of any element in the conjugacy class $s^{\prime}$ corresponding to $\phi^{\prime}$ is contained in $\mathrm{GL}_{n^{\prime}}\left(\mathbb{F}^{\prime}\right)$.

Of course we have a similar diagram if we replace "inert" by " $\ell$-inert" on the left side, and restrict to conjugacy classes with order prime to $\ell$ on the right side.

1.2.6 Integral Deligne-Lusztig theory. - If we embed $\mathrm{GL}_{n^{\prime}}\left(\mathbb{F}^{\prime}\right)$ in $\mathrm{GL}_{n}(\mathbb{F})$ as above, the former group is the group of rational points of an algebraic $\mathbb{F}$-subgroup of $\mathrm{GL}_{n}$ that becomes isomorphic to the Levi subgroup $\mathrm{GL}_{n^{\prime}}^{f}$ over $\overline{\mathbb{F}}$. In this situation, any choice of a parabolic subgroup of $\mathrm{GL}_{n}$ with Levi subgroup $\mathrm{GL}_{n^{\prime}}^{f}$ gives rise to a Deligne-Lusztig variety $Y$ over $\overline{\mathbb{F}}$ with a left action of $\mathrm{GL}_{n}(\mathbb{F})$ and a right action of $\mathrm{GL}_{n^{\prime}}\left(\mathbb{F}^{\prime}\right)$.

Now, fix a conjugacy class $s^{\prime}$ of $\ell^{\prime}$-order in $\mathrm{GL}_{n^{\prime}}\left(\mathbb{F}^{\prime}\right)$, denote by $s$ the image conjugacy class in $\mathrm{GL}_{n}(\mathbb{F})$, and suppose that $C_{\mathrm{GL}_{n}(\mathbb{F})}\left(s^{\prime}\right) \subset \mathrm{GL}_{n^{\prime}}\left(\mathbb{F}^{\prime}\right)$.

The main result of [2] is that, in this setting, the cohomology space $H_{c}^{\operatorname{dim}}\left(Y, \overline{\mathbb{Z}}_{\ell}\right) e_{s^{\prime}}$ induces a Morita equivalence between $e_{s} \overline{\mathbb{Z}}_{\ell} \mathrm{GL}_{n}(\mathbb{F})$ and $e_{s^{\prime}} \overline{\mathbb{Z}}_{\ell} \mathrm{GL}_{n^{\prime}}\left(\mathbb{F}^{\prime}\right)$. All the necessary details are recalled in part 2 .

1.2.7 A Morita equivalence for "Hecke algebras". - Now, the strategy to exhibit an equivalence of categories in the setting of 1.1.4 ii) should be clear. We will construct a Morita equivalence between the Hecke "algebras" $e_{s} \overline{\mathbb{Z}}_{\ell}[\mathbb{A} / \overline{\mathbb{G}}]$ and $e_{s^{\prime}} \overline{\mathbb{Z}}_{\ell}\left[\mathbb{A}^{\prime} / \overline{\mathbb{G}}^{\prime}\right]$ relative to $(\mathbb{F}, n, s)$, resp. $\left(\mathbb{F}^{\prime}, n^{\prime}, s^{\prime}\right)$. Such an equivalence will be given by a "bimodule" in the sense of A.1.6, i.e. a certain functor $\left[\mathbb{A}^{\prime} / \overline{\mathbb{G}}^{\prime}\right]^{\text {opp }} \times[\mathbb{A} / \overline{\mathbb{G}}] \longrightarrow \overline{\mathbb{Z}}_{\ell^{-}}$Mod.

In order to construct this bimodule, we first embed $\iota:\left[\mathbb{A}^{\prime} / \overline{\mathbb{G}}^{\prime}\right] \hookrightarrow[\mathbb{A} / \overline{\mathbb{G}}]$. This requires some choices, among which that of an $\mathbb{F}$-basis of $\mathbb{F}^{\prime}$. The value on a pair $\left(\sigma^{\prime}, \iota \sigma^{\prime}\right)$ of the desired "bimodule" will be given by some cohomology space $\mathcal{P}_{\sigma^{\prime}}^{0}$ as in the previous paragraph ; in particular it will induce a Morita equivalence between $e_{\iota \sigma^{\prime}}^{s} \overline{\mathbb{Z}}_{\ell}\left[\overline{\mathbb{G}}_{\iota \sigma^{\prime}}\right]$ and $e_{\sigma^{\prime}}^{s^{\prime}} \overline{\mathbb{Z}}_{\ell}\left[\overline{\mathbb{G}}_{\sigma^{\prime}}\right]$. The problem is then to extend this assignment to all pairs $\left(\sigma^{\prime}, \sigma\right)$ and make it into a functor.

This turned out to be a more subtle matter, and was only solved after we proved in [1] a property of invariance of the Deligne-Lusztig cohomology under certain change of parabolic subgroups. The heart of this paper is therefore the construction of this "bimodule", which is explained in 4.3.7 after some crucial step in Proposition 2.2.5. Once the construction is done, the fact that it induces a Morita equivalence and preserves the cartesian property is comparatively easier.

1.2.8 Organisation of the paper. - Section 2 reviews the needed input from DeligneLusztig theory. It is mostly expository, with the exception of Proposition 2.2.5 which is a crucial technical point to be used in section 4 . Section 3 deals in detail with the content of 1.2.3 above. Section 4 is the heart of this paper. In particuler, subsection 4.2 explains 1.2.4, while subsection 4.3 contains the construction of a Morita equivalence in the setting of an unramified automorphic induction. Some generalities about "rings with many objects" are postponed to the appendix. 


\section{Finite linear groups}

In this part, $\mathbb{F}=\mathbb{F}_{q}$ denotes a finite field of characteristic $p$, and $\overline{\mathbb{F}}$ an algebraic closure of it.

\subsection{Review of Lusztig theory}

Bold letters $\mathbf{G}, \mathbf{L}, \mathbf{P}$, etc. will typically denote algebraic groups over $\overline{\mathbb{F}}$. A $\mathbb{F}$-structure on such a group gives rise to a "Frobenius isogeny" typically denoted by $F$, such that $\mathbb{F}$-rational points are given, e.g. by $G:=\mathbf{G}(\mathbb{F})=\mathbf{G}^{F}$.

2.1.1 Varieties and functors. - Let $\mathbf{G}$ be a reductive group over $\overline{\mathbb{F}}$ with a $\mathbb{F}$-rational structure. Assume given a parabolic subgroup $\mathbf{P}$ with unipotent radical $\mathbf{U}$, and assume that $\mathbf{P}$ contains an $F$-stable Levi subgroup $\mathbf{L}$. The generalized Deligne-Lusztig variety associated to these data is defined by

$$
Y_{\mathbf{P}}:=\left\{g \mathbf{U} \in \mathbf{G} / \mathbf{U}, g^{-1} F(g) \in \mathbf{U} \cdot F(\mathbf{U})\right\}
$$

This is a variety over $\overline{\mathbb{F}}$ with a left action of $G=\mathbf{G}^{F}$ given by $(\gamma, g \mathbf{U}) \mapsto \gamma g \mathbf{U}$ and a right action of $L=\mathbf{L}^{F}$ given by $(g \mathbf{U}, \delta) \mapsto g \delta \mathbf{U}$. The cohomology complex $R \Gamma_{c}\left(Y_{\mathbf{P}}, \overline{\mathbb{Z}}_{\ell}\right)$ is thus a complex of $\left(\overline{\mathbb{Z}}_{\ell}[G], \overline{\mathbb{Z}}_{\ell}[L]\right)$-bimodules and induces two adjoint functors

$$
\begin{aligned}
& \mathcal{R}_{\mathbf{L} \subset \mathbf{P}}^{\mathbf{G}}=R \Gamma_{c}\left(Y_{\mathbf{P}}, \overline{\mathbb{Z}}_{\ell}\right) \otimes_{\overline{\mathbb{Z}}_{\ell} L}^{L}-: D^{b}\left(\overline{\mathbb{Z}}_{\ell} L\right) \longrightarrow D^{b}\left(\overline{\mathbb{Z}}_{\ell} G\right) \\
& { }^{*} \mathcal{R}_{\mathbf{L} \subset \mathbf{P}}^{\mathbf{G}}: R \operatorname{Hom}_{\overline{\mathbb{Z}}_{\ell} G}\left(R \Gamma_{c}\left(Y_{\mathbf{P}}, \overline{\mathbb{Z}}_{\ell}\right),-\right): D^{b}\left(\overline{\mathbb{Z}}_{\ell} G\right) \longrightarrow D^{b}\left(\overline{\mathbb{Z}}_{\ell} L\right)
\end{aligned}
$$

called "Lusztig's twisted induction", resp. "restriction", and where $D^{b}$ means "bounded derived category". These functors satisfy some rather obvious transitivity properties, and when $\mathbf{P}$ is also $F$-stable, they coincide with Harish-Chandra's usual parabolic functors.

2.1.2 Duals and characters. - Let us fix compatible systems of roots of unity $\iota$ : $(\mathbb{Q} / \mathbb{Z})_{p^{\prime}} \stackrel{\sim}{\longrightarrow} \overline{\mathbb{F}}_{q}^{\times}$and $\jmath:(\mathbb{Q} / \mathbb{Z})_{p^{\prime}} \hookrightarrow \overline{\mathbb{Z}}_{\ell}^{\times}$as in 1.2.1. If $\mathbf{T}$ is a torus defined over $\mathbb{F}$ and $\mathbf{T}^{*}$ denotes the dual torus, also defined over $\mathbb{F}$, there is a group isomorphism $\mathbf{T}^{* F} \stackrel{\sim}{\longrightarrow} \operatorname{Hom}\left(\mathbf{T}^{F}, \overline{\mathbb{F}}^{\times}\right)$ associated to $\iota$, see [3, (8.14)]. Composing with $\jmath \circ \iota^{-1}$ gives a bijection $\mathbf{T}^{* F} \stackrel{\sim}{\longrightarrow} \operatorname{Hom}\left(T, \overline{\mathbb{Z}}_{\ell}^{\times}\right)$. In other words, characters of $T$ valued in $\overline{\mathbb{Z}}_{\ell}$ are parametrized by rational elements $s$ in the dual torus $\mathbf{T}^{*}$. More generally, let us denote by $\mathbf{G}^{*}$ the dual reductive group of $\mathbf{G}$, which is also defined over $\mathbb{F}$. Then any rational element $s$ in the maximal central torus of $\mathbf{G}^{*}$ determines a character $\hat{s}: G \longrightarrow \overline{\mathbb{Z}}_{\ell}^{\times}$.

2.1.3 Rational series. - Let $s$ be a semi-simple conjugacy class in $G^{*}:=\mathbf{G}^{* F}$. An irreducible representation $\pi \in \operatorname{Irr}_{\overline{\mathbb{Q}}_{\ell}}(G)$ is said to belong to the rational series attached to $s$ if there is an $F$-stable torus $\mathbf{T}$ in $\mathbf{G}$, an element $t \in \mathbf{T}^{* F}$ that belongs to $s$, and a Borel subgroup $\mathbf{B}$ containing $\mathbf{T}$ such that $\pi$ occurs with non-zero multiplicity in $\left[\mathcal{R}_{\mathbf{T} \subset \mathbf{B}}^{\mathbf{G}}(\hat{t})\right]$ (here brackets indicate that we consider the image of the complex in the Grothendieck group). We'll denote by $\mathcal{E}(G, s)$ the set of such rational series and by $e_{s}^{G}=e_{s, \overline{\mathbb{Q}}_{\ell}}^{G} \in \overline{\mathbb{Q}}_{\ell}[G]$ the central idempotent that cuts out these rational series. We then have the following remarkable properties : 
i) the subsets $\mathcal{E}(G, s)$ form a partition of $\operatorname{Irr}_{\overline{\mathbb{Q}}_{\ell}}(G)$, and consequently we have the equality $1=\sum_{s} e_{s}^{G}$ in $\overline{\mathbb{Q}}_{\ell}[G]$, see [3, Thm 8.24].

ii) if $s$ consists of $\ell^{\prime}$-elements, then the idempotent $e_{s, \bar{Z}_{\ell}}^{G}:=\sum_{s^{\prime} \sim_{\ell} s} e_{s^{\prime}}^{G}$, where $s^{\prime} \sim_{\ell} s$ means that $s$ is the $\ell$-regular part of $s^{\prime}$, belongs to $\overline{\mathbb{Z}}_{\ell}[G]$, see [2, Thm. A' and Rem. 11.3].

We will denote by $\mathcal{E}_{\ell}(G, s)$ the set of simple modules of $\overline{\mathbb{Z}}_{\ell}[G] e_{s, \overline{\mathbb{Z}}_{\ell}}^{G}$. The representations in $\mathcal{E}(G, 1)$ are also called unipotent series, and those in $\mathcal{E}_{\ell}(G, 1)$ are sometimes called $\ell$-unipotent. Accordingly, $\overline{\mathbb{Z}}_{\ell}[G] e_{1, \overline{\mathbb{Z}}_{\ell}}^{G}$ will be called the $\ell$-unipotent summand of $\overline{\mathbb{Z}}_{\ell}[G]$.

2.1.4 Compatibility with induction functors. - Suppose $\mathbf{L}$ is an F-stable Levi subgroup contained in a parabolic subgroup $\mathbf{P}$ of $\mathbf{G}$. Any semisimple conjugacy class $t$ in $L^{*}$ gives rise to a semisimple class $s$ in $G^{*}$. The map $t \mapsto s$ is finite to one.

From transitivity of twisted induction, we see that

$$
\left[R \Gamma_{c}\left(Y_{\mathbf{P}}, \overline{\mathbb{Q}}_{\ell}\right) e_{t}^{L}\right]=\left[e_{s}^{G} R \Gamma_{c}\left(Y_{\mathbf{P}}, \overline{\mathbb{Q}}_{\ell}\right) e_{t}^{L}\right] .
$$

For the same reason, we have $\left[e_{s}^{G} R \Gamma_{c}\left(Y_{\mathbf{P}}, \overline{\mathbb{Q}}_{\ell}\right) e_{t^{\prime}}^{L}\right]=0$ if $t^{\prime}$ is not contained in $s$, and therefore, putting $e_{s}^{L}:=\sum_{t \mapsto s} e_{t}^{L}$, we get that

$$
\left[R \Gamma_{c}\left(Y_{\mathbf{P}}, \overline{\mathbb{Q}}_{\ell}\right) e_{s}^{L}\right]=\left[e_{s}^{G} R \Gamma_{c}\left(Y_{\mathbf{P}}, \overline{\mathbb{Q}}_{\ell}\right)\right] .
$$

In the particular case that $\mathbf{P}$ itself is $F$-stable, and denoting by $e_{U}$ the idempotent which averages along the group $U=\mathbf{U}^{F}$ of rational points of the radical $\mathbf{U}$ of $\mathbf{P}$, we get the equalities

$$
e_{s}^{G} e_{U}=e_{s}^{G} e_{U} e_{s}^{L}=e_{U} e_{s}^{L}=: e_{s}^{P},
$$

showing in particular that $e_{s}^{G} e_{U}$ is a central idempotent in $\overline{\mathbb{Q}}_{\ell}[P]$. In the same way, when $s$ consists of $\ell^{\prime}$-elements, we have equalities

$$
e_{s, \overline{\mathbb{Z}}_{\ell}}^{G} e_{U}=e_{s, \overline{\mathbb{Z}}_{\ell}}^{G} e_{U} e_{s, \overline{\mathbb{Z}}_{\ell}}^{L}=e_{U} e_{s, \overline{\mathbb{Z}}_{\ell}}^{L}=: e_{s, \overline{\mathbb{Z}}_{\ell}}^{P}
$$

which show that $e_{s, \overline{\mathbb{Z}}_{\ell}}^{G} e_{U}$ is a central idempotent in $\overline{\mathbb{Z}}_{\ell}[P]$.

2.1.6 Morita equivalences. - As above, let $\mathbf{L}$ be an $F$-stable Levi subgroup contained in a parabolic subgroup $\mathbf{P}$ of $\mathbf{G}$. We choose an $F^{*}$-stable embedding $\mathbf{L}^{*} \hookrightarrow \mathbf{G}^{*}$ dual to $\mathbf{L} \hookrightarrow \mathbf{G}$. Let $t$ be a semisimple class in $L^{*}$ and $s$ its image in $G^{*}$. Let also $\Lambda$ denote either $\overline{\mathbb{Q}}_{\ell}$ or $\overline{\mathbb{Z}}_{\ell}$, and suppose that the order of an element of $t$ is invertible in $\Lambda$. The following result is due to Lusztig [7, Prop 6.6] for $\Lambda=\overline{\mathbb{Q}}_{\ell}$ and to Bonnafé and Rouquier [2, Thm B'] for $\Lambda=\overline{\mathbb{Z}}_{\ell}$.

Fact. - Assume that $\mathbf{L}^{*}$ contains the centralizer $C_{\mathbf{G}^{*}}(t)$ of any element in $t$. Then the complex $R \Gamma_{c}\left(Y_{\mathbf{P}}, \Lambda\right) e_{t, \Lambda}^{L}$ is concentrated in degree $d=\operatorname{dim}\left(Y_{\mathbf{P}}\right)$ and the $\left.\left(\Lambda[G] e_{s, \Lambda}^{G}, \Lambda[L] e_{s, \Lambda}^{L}\right)\right)$ bimodule $H_{c}^{d}\left(Y_{\mathbf{P}}, \Lambda\right) e_{t, \Lambda}^{L}$ induces a Morita equivalence, in the sense that the two adjoint functors

$$
\begin{aligned}
\Lambda[L] e_{t, \Lambda}^{L}-\operatorname{Mod} & \rightarrow \Lambda[G] e_{s, \Lambda}^{G}-\operatorname{Mod} \\
M & \mapsto H_{c}^{d}\left(Y_{\mathbf{P}}, \Lambda\right) e_{t, \Lambda}^{L} \otimes_{\Lambda[L]} M \\
\Lambda[G] e_{s, \Lambda}^{G}-\operatorname{Mod} & \rightarrow \Lambda[L] e_{t, \Lambda}^{L}-\operatorname{Mod} \\
N & \mapsto \operatorname{Hom}_{\Lambda[G]}\left(H_{c}^{d}\left(Y_{\mathbf{P}}, \Lambda\right) e_{t, \Lambda}^{L}, N\right)
\end{aligned}
$$

are equivalences of categories. 
In particular these functors induce bijections from $\mathcal{E}(L, t)$ to $\mathcal{E}(G, s)$, and from $\mathcal{E}_{\ell}(L, t)$ to $\mathcal{E}_{\ell}(G, s)$ when appropriate. Note that in the case $\Lambda=\overline{\mathbb{Q}}_{\ell}$, the Morita equivalence follows from this bijection property, but of course things are much more complicated for $\Lambda=\overline{\mathbb{Z}}_{\ell}$.

Note also that in the case where $\mathbf{L}^{*}=C_{\mathbf{G}^{*}}(t)$, the element $t$ provides a character $\hat{t}$ of $L$, and twisting by $\hat{t}$ induces an isomorphism between $\overline{\mathbb{Z}}_{\ell}[L] e_{1, \overline{\mathbb{Z}}_{\ell}}^{L}$ and $\overline{\mathbb{Z}}_{\ell}[L] e_{t, \overline{\mathbb{Z}}_{\ell}}^{L}$, and a bijection $\mathcal{E}(L, t) \mapsto \mathcal{E}(L, 1)$. Therefore, for groups $\mathbf{G}$ such that all centralizers of semisimple elements in $\mathbf{G}^{*}$ are Levi subgroups, these equivalences reduce the study of $\mathcal{E}(G, s)$ and $\overline{\mathbb{Z}}_{\ell}[G] e_{s, \overline{\mathbb{Z}}_{\ell}}^{G}$ to that of $\mathcal{E}\left(C_{\mathbf{G}^{*}}(s)^{*}, 1\right)$ and $\overline{\mathbb{Z}}_{\ell}\left[C_{\mathbf{G}^{*}}(s)^{*}\right] e_{1, \overline{\mathbb{Z}}_{\ell}}^{G}$.

2.1.7 Dependence on the parabolic subgroup. - In our later application to p-adic groups, it will be important to know that the equivalences of 2.1.6 are "naturally independent" of the parabolic sugroup $\mathbf{P}$ that contains $\mathbf{L}$ as a Levi factor. Note that this is far from obvious since even the dimension of $Y_{\mathbf{P}}$ depends on $\mathbf{P}$.

We need here an apparently more general notion of "Deligne-Lusztig varieties". Namely, start with a collection $\mathbf{P}_{1}, \cdots, \mathbf{P}_{r}$ of parabolic subgroups with Levi component $\mathbf{L}$, and define

$$
Y_{\mathbf{P}_{1}, \cdots, \mathbf{P}_{r}}:=\left\{\begin{array}{c}
\left(g_{1} \mathbf{U}_{1}, \cdots, g_{r} \mathbf{U}_{r}\right) \in \mathbf{G} / \mathbf{U}_{1} \times \cdots \times \mathbf{G} / \mathbf{U}_{r} \text { such that } \\
g_{i}^{-1} g_{i+1} \in \mathbf{U}_{i} \cdot \mathbf{U}_{i+1} \text { for } i=1, \cdots, r \text {, and } \\
g_{r}^{-1} F\left(g_{1}\right) \in \mathbf{U}_{r} \cdot F\left(\mathbf{U}_{1}\right)
\end{array}\right\}
$$

This is again a variety over $\overline{\mathbb{F}}$ with a left action of $\mathbf{G}^{F}$ given by $\left(\gamma,\left(g_{1} \mathbf{U}_{1}, \cdots, g_{r} \mathbf{U}_{r}\right)\right) \mapsto$ $\left(\gamma g_{1} \mathbf{U}_{1}, \cdots, \gamma g_{r} \mathbf{U}_{r}\right)$ and a right action of $\mathbf{L}^{F}$ given by $\left(\left(g_{1} \mathbf{U}_{1}, \cdots, g_{r} \mathbf{U}_{r}\right), \delta\right) \mapsto\left(g_{1} \delta \mathbf{U}_{1}, \cdots, g_{r} \delta \mathbf{U}_{r}\right)$.

Now let $\mathbf{P}, \mathbf{P}^{\prime}$ be two parabolic subgroups with Levi component $\mathbf{L}$. Consider the closed subvariety

$$
\mathcal{Y}_{\mathbf{P}, \mathbf{P}^{\prime}}:=\left\{\left(g \mathbf{U}, g^{\prime} \mathbf{U}^{\prime}\right) \in Y_{\mathbf{P}, \mathbf{P}^{\prime}}, g^{-1} F(g) \in \mathbf{U} \cdot{ }^{F} \mathbf{U}\right\} .
$$

We have a diagram

$$
Y_{\mathbf{P}, \mathbf{P}^{\prime}} \stackrel{\iota}{\longrightarrow} \mathcal{Y}_{\mathbf{P}, \mathbf{P}^{\prime}} \stackrel{\pi}{\longrightarrow} Y_{\mathbf{P}}
$$

where $\pi$ is the first projection $\left(g \mathbf{U}, g^{\prime} \mathbf{U}^{\prime}\right) \mapsto g \mathbf{U}$. It follows from [1, Corollary 5.3] that $\pi$ is an affine bundle of relative dimension $\delta:=\frac{1}{2}\left(\operatorname{dim}\left(Y_{\mathbf{P}, \mathbf{P}^{\prime}}\right)-\operatorname{dim}\left(Y_{\mathbf{P}}\right)\right)$. Therefore the map $\pi_{\text {! }}$ induces an isomorphism $R \Gamma_{c}\left(\mathcal{Y}_{\mathbf{P}, \mathbf{P}^{\prime}}, \overline{\mathbb{Z}}_{\ell}\right) \stackrel{\sim}{\longrightarrow} R \Gamma_{c}\left(Y_{\mathbf{P}}, \overline{\mathbb{Z}}_{\ell}\right)[-2 \delta](-\delta)$. Composing with $\iota$ ! we get in particular a morphism

$$
\Psi_{\mathbf{P}, \mathbf{P}^{\prime}}^{(2)}: R \Gamma_{c}^{\operatorname{dim}}\left(Y_{\mathbf{P}, \mathbf{P}^{\prime}}, \overline{\mathbb{Z}}_{\ell}\right) \longrightarrow R \Gamma_{c}^{\operatorname{dim}}\left(Y_{\mathbf{P}}, \overline{\mathbb{Z}}_{\ell}\right)
$$

where we use the notation $R \Gamma_{c}^{\operatorname{dim}}\left(Y, \overline{\mathbb{Z}}_{\ell}\right):=R \Gamma_{c}\left(Y, \overline{\mathbb{Z}}_{\ell}\right)[\operatorname{dim}(Y)]\left(\frac{1}{2} \operatorname{dim}(Y)\right)$ for a smooth variety $Y$ of pure dimension. In the same way we get a morphism $\Psi_{\mathbf{P}^{\prime}, F(\mathbf{P})}^{(2)}$. Since the morphism of varieties $Y_{\mathbf{L} \subset \mathbf{P}, \mathbf{P}^{\prime}}^{\mathbf{G}} \longrightarrow Y_{\mathbf{L} \subset \mathbf{P}^{\prime}, F(\mathbf{P})}^{\mathbf{G}}$ defined by $\left(g \mathbf{U}, g^{\prime} \mathbf{U}^{\prime}\right) \mapsto\left(g^{\prime} \mathbf{U}^{\prime}, F(g \mathbf{U})\right)$ is known to induce an equivalence of étale sites, we see that $\Psi_{\mathbf{P}^{\prime}, F(\mathbf{P})}^{(2)}$ provides a morphism

$$
\Psi_{\mathbf{P}, \mathbf{P}^{\prime}}^{(1)}: R \Gamma_{c}^{\operatorname{dim}}\left(Y_{\mathbf{P}, \mathbf{P}^{\prime}}, \overline{\mathbb{Z}}_{\ell}\right) \longrightarrow R \Gamma_{c}^{\operatorname{dim}}\left(Y_{\mathbf{P}^{\prime}}, \overline{\mathbb{Z}}_{\ell}\right) .
$$

Let us now choose a $F$-compatible embedding $\mathbf{L}^{*} \hookrightarrow \mathbf{G}^{*}$. We can transfer $\mathbf{P}$ and $\mathbf{P}^{\prime}$ to dual parabolic subgroups $\mathbf{P}^{*}$ and $\mathbf{P}^{\prime *}$ with Levi component $\mathbf{L}^{*}$. The following result follows from Theorem 6.2 of $[1]$.

\footnotetext{
${ }^{2}$ The reader will find in 4 a shorter account of this result, with notation closer to those of this article
} 
Fact. - Let $\Lambda$ denote either $\overline{\mathbb{Z}}_{\ell}$ or $\overline{\mathbb{Q}}_{\ell}$. Let $t$ be a semisimple element in $L^{*}$ with order invertible in $\Lambda$. Assume that $C_{\mathbf{G}^{*}}(t) \cap \mathbf{P}^{*}=C_{\mathbf{G}^{*}}(t) \cap \mathbf{P}^{\prime *}$. Then the morphisms $\Psi_{\mathbf{P}_{,} \mathbf{P}^{\prime}}^{(2)}$ and $\Psi_{\mathbf{P}, \mathbf{P}^{\prime}}^{(1)}$ induce isomorphisms

$$
R \Gamma_{c}^{\operatorname{dim}}\left(Y_{\mathbf{P}}, \Lambda\right) e_{t, \Lambda}^{L} \stackrel{\sim}{\sim} R \Gamma_{c}^{\operatorname{dim}}\left(Y_{\mathbf{P}, \mathbf{P}^{\prime}}, \Lambda\right) e_{t, \Lambda}^{L} \stackrel{\sim}{\longrightarrow} R \Gamma_{c}^{\operatorname{dim}}\left(Y_{\mathbf{P}^{\prime}}, \Lambda\right) e_{t, \Lambda}^{L} .
$$

2.1.10 General linear groups. - We assume here that $G$ is a product of general linear groups over extensions of $\mathbb{F}$. Then we have the following additional properties :

i) $\mathcal{E}(G, s)$ contains a cuspidal representation if and only if $s$ is elliptic in $G^{*}$ (by which we mean that $s$ does not intersect any rational parabolic subgroup). Moreover in this case, $\mathcal{E}(G, s)$ consists of one element, so that we get a bijection

\{elliptic conjugacy classes in $\left.G^{*}\right\} \leftrightarrow\left\{\right.$ classes of cuspidal irreps of $G$ over $\left.\overline{\mathbb{Q}}_{\ell}\right\}$.

To see why these properties hold, note first that if $s$ is elliptic then for any rational parabolic subgroup $P$ we have $e_{s}^{G} e_{U_{P}}=0$ by (2.1.5), thus $\mathcal{E}(G, s)$ consists of cuspidal representations. Picking up an irrep in each $\mathcal{E}(G, s)$ we thus get an injection from the set of elliptic conjugacy classes to that of cuspidal representations. But a counting argument based on Green's classification shows that we get all cuspidals this way.

ii) If $s$ is not elliptic, there is a rational parabolic subgroup in $\mathbf{G}$ whose dual intersects $s$. Choose $\mathbf{P}$ minimal for this property, and let $\mathbf{L}$ be a Levi complement and $t \in \mathbf{L}^{*} \cap s$. Thus $t$ is elliptic in $L^{*}$ and corresponds to a cuspidal irrep $\sigma$ of $L$ by i) above. We get in this way a bijection $s \mapsto[L, \sigma]$

$\left\{\mathrm{s} /\right.$ simple conjugacy classes in $\left.G^{*}\right\} \leftrightarrow\left\{G\right.$-conj. classes of cuspidal pairs $(L, \sigma)$ over $\left.\overline{\mathbb{Q}}_{\ell}\right\}$.

Moreover, by the compatibility with induction of paragraph 2.1.4, $\mathcal{E}(G, s)$ contains all irreducible subquotients of $\mathcal{R}_{\mathbf{L} \subset \mathbf{P}}^{\mathbf{G}}(\sigma)=\operatorname{Ind}_{P}^{G}(\sigma)$. But then, comparing the partition of $\operatorname{Irr}_{\overline{\mathbb{Q}}_{\ell}}(G)$ in rational series, and that given by the cuspidal support, we see that

$$
\mathcal{E}(G, s)=\operatorname{JH}\left(\operatorname{Ind}_{P}^{G}(\sigma)\right)
$$

iii) Assume now that $s$ consists of $\ell^{\prime}$-elements. Then $\mathcal{E}_{\ell}(G, s)$ contains a supercuspidal representation if and only if $s$ is elliptic in $G^{*}$. Moreover in this case, $\mathcal{E}_{\ell}(G, s)$ consists of one element, so that we get a bijection

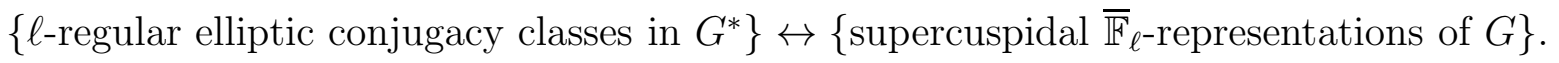

To see these properties, observe first that since the centralizer of an elliptic element is a torus, the Morita equivalence of paragraph 2.1.6 shows that $\mathcal{E}_{\ell}(G, s)$ consists of one representation, which by the way is cuspidal. If it were not supercuspidal, it would occur as a subquotient of some induced representation hence would belong to some $\mathcal{E}_{\ell}\left(G, s^{\prime}\right)$ with $s^{\prime}$ not elliptic, a contradiction. Thus we get an injective map from $\ell$-regular elliptic classes to supercuspidals. Again a counting argument, relying this time on James' classification, shows it is also surjective. 
iv) If $s$ is not elliptic, choose $\mathbf{P}$ minimal among rational parabolic subgroups whose dual intersects $s$, and choose $\mathbf{L}$ a rational Levi complement and $t \in \mathbf{L}^{*} \cap s$. Thus $t$, being elliptic and $\ell$-regular in $L^{*}$, corresponds to a supercuspidal irrep $\sigma$ of $L$ over $\overline{\mathbb{F}}_{\ell}$. We get in this way a bijection

$\left\{\mathrm{s} /\right.$ simple $\ell$-regular conjugacy classes in $\left.G^{*}\right\}$

$\leftrightarrow\left\{G\right.$-conj. classes of supercuspidal pairs $(L, \sigma)$ over $\left.\overline{\mathbb{F}}_{\ell}\right\}$.

Moreover, by the compatibility with induction of paragraph 2.1.4, $\mathcal{E}_{\ell}(G, s)$ contains all irreducible subquotients of $\mathcal{R}_{\mathbf{L} \subset \mathbf{P}}^{\mathbf{G}}(\sigma)=\operatorname{Ind}_{P}^{G}(\sigma)$. But then, comparing the partition of $\operatorname{Irr}_{\overline{\mathbb{F}}_{\ell}}(G)$ as a union of $\mathcal{E}_{\ell}(G, s)$, and that given by the supercuspidal support, we see that

$$
\mathcal{E}_{\ell}(G, s)=\operatorname{JH}\left(\operatorname{Ind}_{P}^{G}(\sigma)\right)
$$

\subsection{General linear groups in a coordinate-free setting}

The setting here is the following. Let $V$ be a vector space over $\mathbb{F}$ of finite dimension $n$. We put $G:=\operatorname{Aut}_{\mathbb{F}}(V)$ and we also denote by $\mathbf{G}$ the corresponding algebraic group over $\overline{\mathbb{F}}$ and $F$ the Frobenius associated with the $\mathbb{F}$-structure, so that $G=\mathbf{G}^{F}$. Further, let $\mathbb{F}^{\prime}$ be a finite subfield of $\overline{\mathbb{F}}$ containing $\mathbb{F}$, and let $n^{\prime}, V^{\prime}, G^{\prime}, \mathbf{G}^{\prime}, F^{\prime}$ be as above, but relative to $\mathbb{F}^{\prime}$. We assume that $|\mathbb{F}|^{n}=\left|\mathbb{F}^{\prime}\right|^{n^{\prime}}$. We may then see $G^{\prime}$ as the rational points of some Levi subgroup in $\mathbf{G}$, and thus use the theory of the previous section to go from representations of $G^{\prime}$ to representations of $G$.

2.2.1 Varieties. - Fix an $\mathbb{F}$-linear isomorphism $\iota: V^{\prime} \stackrel{\sim}{\longrightarrow} V$. This induces a group embedding $\iota_{*}: G^{\prime} \hookrightarrow G$, which comes as the $F$-fixed part of an embedding $\iota_{*}: \operatorname{Res}_{\mathbb{F}^{\prime} \mid \mathbb{F}} \mathbf{G}^{\prime} \hookrightarrow \mathbf{G}$ of algebraic groups over $\mathbb{F}$. The image of this embedding is a Levi subgroup $\mathbf{L}_{\iota}$ of $\mathbf{G}$ such that $\mathbf{L}_{\iota}^{F}=\iota_{*}\left(G^{\prime}\right)$. More precisely, on the $\overline{\mathbb{F}}$-vector space $\bar{V}:=\overline{\mathbb{F}} \otimes_{\mathbb{F}} V$, we get from $\iota_{*}$ a $\mathbb{F}$-linear action of the field $\mathbb{F}^{\prime}$. Thanks to this action, we have a direct sum decomposition

$$
\bar{V}=\bigoplus_{i=0}^{e-1} \bar{V}_{i}, \text { with } \bar{V}_{i}=\left\{v \in \bar{V}, \forall a \in \mathbb{F}^{\prime}, \iota_{*}(a) v=F^{i}(a) v\right\},
$$

and this identifies $\mathbf{L}_{\iota}$ with $\prod_{i} \operatorname{Aut}_{\overline{\mathbb{F}}}\left(\bar{V}_{i}\right)$. We denote by $\mathbf{P}_{\iota}$ the parabolic subgroup with Levi $\mathbf{L}_{\iota}$ associated to the flag

$$
\bar{V} \supset \bigoplus_{i \geqslant 1} \bar{V}_{i} \supset \bigoplus_{i \geqslant 2} \bar{V}_{i} \supset \cdots \supset\{0\}
$$

Unlike $\mathbf{L}_{\iota}$, this parabolic subgroup is only defined over $\mathbb{F}^{\prime}$, not over $\mathbb{F}$. Let $\mathbf{U}_{\iota}$ be the unipotent radical of $\mathbf{P}_{\iota}$ and consider the variety

$$
Y_{\iota, V^{\prime}}^{V}:=Y_{\mathbf{P}_{\iota}}
$$

It has a right action of $G=\mathbf{G}^{F}$ given by $\left(\gamma, g \mathbf{U}_{\iota}\right) \mapsto \gamma g \mathbf{U}_{\iota}$ and a left action of $G^{\prime}$ given by $\left(g \mathbf{U}_{\iota}, \gamma^{\prime}\right) \mapsto g \iota_{*}\left(\gamma^{\prime}\right) \mathbf{U}_{\iota}$. 
2.2.2 Idempotents and bimodules. - As usual we denote by $\Lambda$ either $\overline{\mathbb{Q}}_{\ell}$ or $\overline{\mathbb{Z}}_{\ell}$. We now fix a semisimple conjugacy class $s^{\prime}$ in $\mathrm{GL}_{n^{\prime}}\left(\mathbb{F}^{\prime}\right)$ with invertible order in $\Lambda$. This determines a conjugacy class of rational elements in $\mathbf{G}^{\prime}$ and thus a central idempotent $e_{s^{\prime}, \Lambda}^{G^{\prime}} \in \Lambda\left[G^{\prime}\right]$. Moreover, choosing an $\mathbb{F}$-basis of $\mathbb{F}^{\prime}$, we get a conjugacy class $s$ in $\mathrm{GL}_{n}(\mathbb{F})$, which does not depend on the choice of this basis. Then, similarly we have an idempotent $e_{s, \Lambda}^{G} \in \Lambda[G]$.

We assume that the centralizer of an element of $s^{\prime}$ in $\mathrm{GL}_{n}(\mathbb{F})$ is contained in $\mathrm{GL}_{n^{\prime}}\left(\mathbb{F}^{\prime}\right)$.

Then, as recalled in Fact 2.1.6, the $\left(\Lambda[G], \Lambda\left[G^{\prime}\right]\right)$-bimodule

$$
\mathcal{P}_{\iota, V^{\prime}}^{V, s^{\prime}}=\mathcal{P}_{\iota, V^{\prime}}^{V, s^{\prime}, \Lambda}:=H_{c}^{\operatorname{dim}}\left(Y_{\iota, V^{\prime}}^{V}, \Lambda\right) e_{s^{\prime}, \Lambda}^{G^{\prime}}
$$

induces a Morita equivalence between $\Lambda\left[G^{\prime}\right] e_{s^{\prime}, \Lambda}^{G^{\prime}}$ and $\Lambda[G] e_{s, \Lambda}^{G}$.

2.2.3 Flags and parabolic induction. - Let $\mathcal{V}^{\prime}=\left(V_{0}^{\prime}=V^{\prime} \supset V_{1}^{\prime} \supset \cdots \supset V_{r}^{\prime}=0\right)$ be a flag of $\mathbb{F}^{\prime}$-subvector spaces in $V^{\prime}$, and let $\mathbf{P}^{\prime} \subset \mathbf{G}^{\prime}$ be the associated parabolic subgroup, with radical $\mathbf{U}^{\prime}$ and Levi quotient $\mathbf{M}^{\prime}$, all $F^{\prime}$-stables. Denote by $\mathcal{V}:=\iota_{*} \mathcal{V}^{\prime}$ its image as a flag in $V$, and let $\mathbf{P} \subset \mathbf{G}, \mathbf{U}, \mathbf{M}$ be similarly defined. We note that, upon identifying $\operatorname{Res}_{\mathbb{F}^{\prime} \mid \mathbb{F}}\left(\mathbf{G}^{\prime}\right)$ to a Levi subgroup of $\mathbf{G}$ through $\iota$, we have the equality $\mathbf{P} \cap \operatorname{Res}_{\mathbb{F}^{\prime} \mid \mathbb{F}}\left(\mathbf{G}^{\prime}\right)=\operatorname{Res}_{\mathbb{F}^{\prime} \mid \mathbb{F}}\left(\mathbf{P}^{\prime}\right)$. Also, $\iota$ identifies $\operatorname{Res}_{\mathbb{F}^{\prime} \mid \mathbb{F}}\left(\mathbf{M}^{\prime}\right)$ to an $F$-stable Levi subgroup of $\mathbf{M}$ and the projection of $\mathbf{P}_{\iota} \cap \mathbf{P}$ to $\mathbf{M}$ provides a parabolic subgroup $\mathbf{P}_{\iota}^{\mathbf{M}}$ of $\mathbf{M}$ with Levi component $\operatorname{Res}_{\mathbb{F}^{\prime} \mid \mathbb{F}} \mathbf{M}^{\prime}$. We then put

$$
Y_{\iota, \mathcal{V}^{\prime}}^{V}:=Y_{\mathbf{P}_{\iota}^{\mathrm{M}}}=\prod_{j} Y_{\iota, V_{j}^{\prime} / V_{j+1}^{\prime}}^{V_{j} / V_{j+1}}
$$

which is a $M \times M^{\prime}$-variety that we will also consider as a $P \times P^{\prime}$-variety (since $\mathbf{P}$ and $\operatorname{Res}\left(\mathbf{P}^{\prime}\right)$ are $F$-stable, we can use the notation $P=\mathbf{P}^{F}$ and $P^{\prime}=\mathbf{P}^{\prime F^{\prime}}$ ). Recall our notation $e_{s^{\prime}}^{P^{\prime}}$ from (2.1.5) and put

$$
\mathcal{P}_{\iota, \mathcal{V}^{\prime}}^{V, s^{\prime}}=\mathcal{P}_{\iota, \mathcal{V}^{\prime}}^{V, s^{\prime}, \Lambda}:=H_{c}^{\operatorname{dim}}\left(Y_{\iota, \mathcal{V}^{\prime}}^{V}, \Lambda\right) e_{s^{\prime}, \Lambda}^{P^{\prime}}
$$

As above, it induces a Morita equivalence between $\Lambda\left[P^{\prime}\right] e_{s^{\prime}, \Lambda}^{P^{\prime}}$ and $\Lambda[P] e_{s^{\prime}, \Lambda}^{P}$ (note that everything boils down to the Levi quotients $M, M^{\prime}$ where the situation is a product of instances of the last paragraph).

Later, we will need the following functoriality property of this parabolic construction. If $\gamma^{\prime} \in G^{\prime}$ with image $\gamma:=\iota_{*}\left(\gamma^{\prime}\right) \in G$, then the conjugation map by $\gamma$ in $\mathbf{G}$

$$
\operatorname{Int}_{\gamma}: Y_{\iota, \mathcal{V}^{\prime}}^{V} \longrightarrow Y_{\iota, \gamma^{\prime}\left(\mathcal{V}^{\prime}\right)}^{V}, m \mathbf{U}_{\iota}^{\mathbf{M}} \mapsto \gamma m \mathbf{U}_{\iota}^{M} \gamma^{-1}
$$

induces an isomorphism of varieties which is equivariant with respect to $\operatorname{Int}_{\gamma} \times \operatorname{Int}_{\gamma^{\prime}}: P \times P^{\prime} \stackrel{\sim}{\longrightarrow}$ ${ }^{\gamma} P \times{ }^{\prime} P^{\prime}$. This induces in turn an isomorphism

$$
\left.\operatorname{Int}_{\gamma, \mathcal{V}^{\prime}}: \mathcal{P}_{\iota, \mathcal{V}^{\prime}}^{V, s^{\prime}} \stackrel{\sim}{\longrightarrow} \mathcal{P}_{\iota, \gamma^{\prime}}^{V, s^{\prime}}\right)
$$

with the same equivariance property. Note that when $\gamma^{\prime} \in P^{\prime}$, this isomorphism is given by the natural action of $\left(\gamma, \gamma^{\prime-1}\right) \in P \times P^{\prime}$ on $Y_{\iota, \mathcal{V}^{\prime}}^{V}$.

The following result is crucial for the main theorem of the paper. 
2.2.5 Proposition. - There exists a family of isomorphisms of $\left(\Lambda P_{2}, \Lambda P_{1}^{\prime}\right)$-bimodules

$$
\phi_{\mathcal{V}_{1}^{\prime}}^{\mathcal{V}_{2}^{\prime}}: \Lambda P_{2} \otimes_{\Lambda P_{1}} \mathcal{P}_{\iota, \mathcal{V}_{1}^{\prime}}^{V, s^{\prime}} \stackrel{\sim}{\longrightarrow} \mathcal{P}_{\iota, \mathcal{V}_{2}^{\prime}}^{V, s^{\prime}} e_{U_{1}^{\prime}}
$$

indexed by pairs of flags $\mathcal{V}_{1}^{\prime}, \mathcal{V}_{2}^{\prime}$ in $V^{\prime}$ such that $\mathcal{V}_{1}^{\prime}$ refines $\mathcal{V}_{2}^{\prime}$ (so that $P_{1} \subset P_{2}$ and $P_{1}^{\prime} \subset P_{2}^{\prime}$ ) and which satisfy the following properties.

i) (functoriality) For any $\gamma^{\prime} \in G^{\prime}$ with image $\gamma=\iota_{*}\left(\gamma^{\prime}\right)$, we have $\phi_{\gamma^{\prime} \mathcal{V}_{1}^{\prime}}^{\gamma^{\prime} \mathcal{V}^{\prime}}=\operatorname{Int}_{\gamma, \mathcal{V}_{2}^{\prime}} \circ \phi_{\mathcal{V}_{1}^{\prime}}^{\mathcal{V}_{2}^{\prime}} \circ \operatorname{Int}_{\gamma, \mathcal{V}_{1}^{\prime}}^{-1}$

ii) (transitivity) If $\mathcal{V}_{3}^{\prime}$ is a coarser flag than $\mathcal{V}_{2}^{\prime}$, then the following diagram commutes :

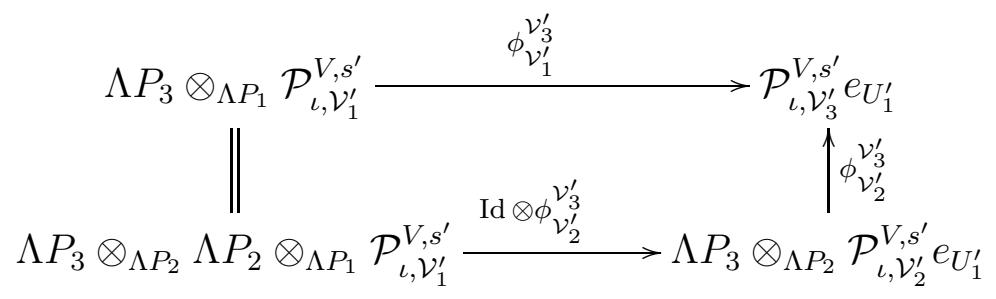

Proof. Step 1. Construction of $\phi_{\mathcal{V}_{1}^{\prime}}^{\mathcal{V}_{1}^{\prime}}$. For the sake of clarity, we first assume that $\mathcal{V}_{2}^{\prime}$ is the trivial flag $V^{\prime}$ and we abbreviate $\mathcal{V}^{\prime}=\mathcal{V}_{1}^{\prime}$. We take up the notation of subsection 2.2.3. Consider the two parabolic subgroups of $\mathbf{G}$

$$
\mathbf{Q}:=\left(\mathbf{P}_{\iota} \cap \mathbf{P}\right) \mathbf{U}_{\iota} \text { and } \mathbf{Q}^{\prime}:=\left(\mathbf{P}_{\iota} \cap \mathbf{P}\right) \mathbf{U}
$$

Both have Levi quotient isomorphic to $\operatorname{Res}_{\mathbb{F}^{\prime} \mid \mathbb{F}}\left(\mathbf{M}^{\prime}\right)$. In fact, putting $\bar{V}_{i, j}:=\bar{V}_{i} \cap \overline{\iota\left(V_{j}^{\prime}\right)}$, the parabolic subgroup $\mathbf{Q}$, resp. $\mathbf{Q}^{\prime}$, corresponds to the flag obtained by ordering pairs $i, j$ according to the lexicographical order on the couple $(i, j)$, resp. on the couple $(j, i)$.

Let us temporarily choose a splitting of the flag $\mathcal{V}^{\prime}$. This gives rise to splittings $\mathbf{M}^{\prime} \hookrightarrow \mathbf{P}^{\prime}$ and $\mathbf{M} \hookrightarrow \mathbf{P}$, that fit in a diagram of Levi and parabolic subgroups

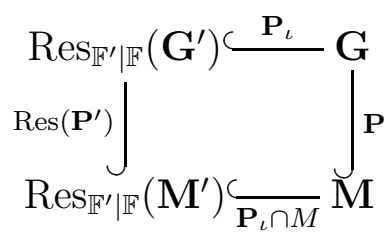

so that $\mathbf{Q}$ corresponds to the upper way of composing parabolic subgroups in this diagram while $\mathrm{Q}^{\prime}$ corresponds to the lower way. A good point about general linear groups is that they are isomorphic to their own duals. In fact the same diagram as above may serve as a dual diagram to itself. Now, let $t^{\prime}$ be a semisimple element in the intersection of our semisimple class $s^{\prime}$ and $M^{\prime}=\mathbf{M}^{\prime *, F^{*}}$. By hypothesis, the centralizer $C_{\mathbf{G}}\left(t^{\prime}\right)$ is contained in $\operatorname{Res}\left(\mathbf{G}^{\prime}\right)$. Moreover, we have $\mathbf{Q} \cap \operatorname{Res}\left(\mathbf{G}^{\prime}\right)=\mathbf{Q}^{\prime} \cap \operatorname{Res}\left(\mathbf{G}^{\prime}\right)=\operatorname{Res}\left(\mathbf{P}^{\prime}\right)$. Therefore we may apply Fact 2.1.7 which provides us with an isomorphism

$$
\Psi_{\mathbf{Q}, \mathbf{Q}^{\prime}}^{(1)} \circ\left(\Psi_{\mathbf{Q}, \mathbf{Q}^{\prime}}^{(2)}\right): R \Gamma_{c}^{\operatorname{dim}}\left(Y_{\mathbf{Q}^{\prime}}, \Lambda\right) e_{t^{\prime}, \Lambda}^{M^{\prime}} \stackrel{\sim}{\longrightarrow} R \Gamma_{c}^{\operatorname{dim}}\left(Y_{\mathbf{Q}}, \Lambda\right) e_{t^{\prime}, \Lambda}^{M^{\prime}} .
$$

Summing on all $t^{\prime}$ as above up to $M^{\prime}$-conjugacy, we get an isomorphism in $D^{b}\left(\Lambda G \otimes_{\Lambda} \Lambda M^{\prime \text { opp }}\right)$

$$
\Phi_{\mathbf{Q}^{\prime} \mid \mathbf{Q}}:=\Psi_{\mathbf{Q}, \mathbf{Q}^{\prime}}^{(1)} \circ\left(\Psi_{\mathbf{Q}, \mathbf{Q}^{\prime}}^{(2)}\right): R \Gamma_{c}^{\operatorname{dim}}\left(Y_{\mathbf{Q}^{\prime}}, \Lambda\right) e_{s^{\prime}, \Lambda}^{M^{\prime}} \stackrel{\sim}{\longrightarrow} R \Gamma_{c}^{\operatorname{dim}}\left(Y_{\mathbf{Q}}, \Lambda\right) e_{s^{\prime}, \Lambda}^{M^{\prime}} .
$$


It is easily checked that these isomorphisms do not depend on the choice of a splitting of $\mathcal{V}^{\prime}$.

Now, the transitivity for DL varieties tells us that the multiplication map induces an isomorphism

$$
Y_{\iota, V^{\prime}}^{V} \times{ }^{G^{\prime}} G^{\prime} / U^{\prime}=Y_{\mathbf{P}_{\iota}} \times{ }^{\operatorname{Res}\left(\mathbf{G}^{\prime}\right)^{F}} Y_{\operatorname{Res}\left(\mathbf{P}^{\prime}\right)} \stackrel{\sim}{\longrightarrow} Y_{\mathbf{Q}} .
$$

Similarly, upon choosing a splitting of the flag $\mathcal{V}^{\prime}$, giving rise to splittings $\mathbf{M}^{\prime} \hookrightarrow \mathbf{P}^{\prime}$ and $\mathbf{M} \hookrightarrow \mathbf{P}$, we get an isomorphism

$$
G \times{ }^{P} Y_{\iota, \mathcal{V}^{\prime}}^{V}=G / U \times{ }^{M} Y_{\iota, \mathcal{V}^{\prime}}^{V}=Y_{\mathbf{P}} \times{ }^{M} Y_{\mathbf{P}_{\iota}^{\mathrm{M}}} \stackrel{\sim}{\longrightarrow} Y_{\mathbf{Q}^{\prime}}
$$

which does not depend on the splitting.

Taking cohomology and applying idempotents, the isomorphism (2.2.7) becomes our desired isomorphism

$$
\phi_{\mathcal{V}^{\prime}}^{V^{\prime}}: \Lambda G \otimes_{\Lambda P} \mathcal{P}_{\iota, \mathcal{V}^{\prime}}^{V, s^{\prime}} \stackrel{\sim}{\longrightarrow} \mathcal{P}_{\iota, V^{\prime}}^{V, s^{\prime}} e_{U^{\prime}}
$$

Let us now treat a general pair $\left(\mathcal{V}_{1}^{\prime}, \mathcal{V}_{2}^{\prime}\right)$ as in the proposition. Let $t^{\prime}$ be any conjugacy class in $P_{2}^{\prime}$ contained in $s^{\prime}$. Then, repeating the above discussion, we get an isomorphism similar to (2.2.8)

$$
\Lambda P_{2} \otimes_{\Lambda P_{1}} H_{c}^{\operatorname{dim}}\left(Y_{\iota, \mathcal{V}_{1}^{\prime}}\right) e_{t^{\prime}, \Lambda}^{P_{1}^{\prime}} \stackrel{\sim}{\longrightarrow} H_{c}^{\operatorname{dim}}\left(Y_{\iota, \mathcal{V}_{2}^{\prime}}\right) e_{t^{\prime}, \Lambda}^{P_{2}^{\prime}} e_{U_{1}^{\prime}} .
$$

Indeed the whole situation is a product of instances of the case just treated. Now, summing over all $t^{\prime}$ provides us with the desired isomorphism

$$
\phi_{\mathcal{V}_{1}^{\prime}}^{\mathcal{V}_{2}^{\prime}}: \Lambda P_{2} \otimes_{\Lambda P_{1}} \mathcal{P}_{\iota, \mathcal{V}_{1}^{\prime}}^{V, s^{\prime}} \stackrel{\sim}{\longrightarrow} \mathcal{P}_{\iota, \mathcal{V}_{2}^{\prime}}^{V, s^{\prime}} e_{U_{1}^{\prime}}
$$

Step 2. Property i). This follows quite clearly from the geometric origin of $\phi_{\mathcal{V}_{1}^{\prime}}^{\mathcal{V}^{\prime}}$. Indeed we have used various geometric relations between DL varieties that all have a clear functorial behavior under conjugacy by $\iota_{*}\left(\gamma^{\prime}\right)$, the most involved point being the isomorphism (2.2.7) whose geometric construction was recalled in subsection 2.1.7.

Step 3. Property ii). To simplify the discussion a bit we will assume that $\mathcal{V}_{3}^{\prime}=V^{\prime}$. As pointed out in Step 1, this is not a real loss of generality. Let us denote by $\mathbf{Q}_{1}$ and $\mathbf{Q}_{1}^{\prime}$ the parabolic subgroups involved in Step 1 and associated to $\mathcal{V}_{1}^{\prime}$. Further, let us introduce a third parabolic subgroup with Levi quotient $\operatorname{Res}\left(\mathbf{M}_{1}^{\prime}\right)$ :

$$
\mathbf{Q}_{1}^{2}:=\left(\mathbf{P}_{\iota} \cap \mathbf{P}_{1}\right)\left(\mathbf{U}_{\iota} \cap \mathbf{P}_{2}\right) \mathbf{U}_{2} .
$$

Upon choosing splittings of the flags $\mathcal{V}_{1}^{\prime}$ and $\mathcal{V}_{2}^{\prime}$, this parabolic subgroup is the "intermediate" way to compose parabolic subgroups in the following diagram

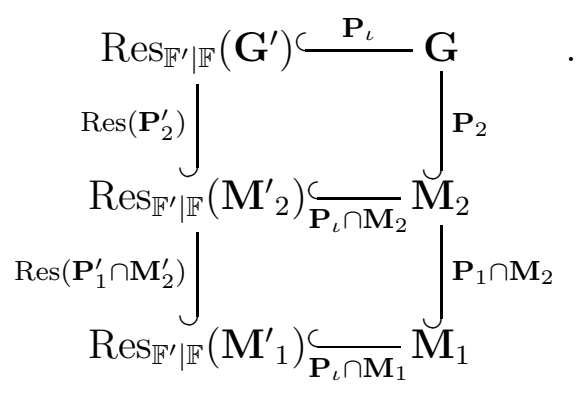


Now, looking at the construction in Step 1, we see that what needs to be proved is the equality $(2.2 .10)$

$$
\Phi_{\mathbf{Q}_{1}^{\prime} \mid \mathbf{Q}_{1}}=\Phi_{\mathbf{Q}_{1}^{2} \mid \mathbf{Q}_{1}} \circ \Phi_{\mathbf{Q}_{1}^{\prime} \mid \mathbf{Q}_{1}^{2}} .
$$

involving isomorphisms of type (2.2.7). Consider the following diagram

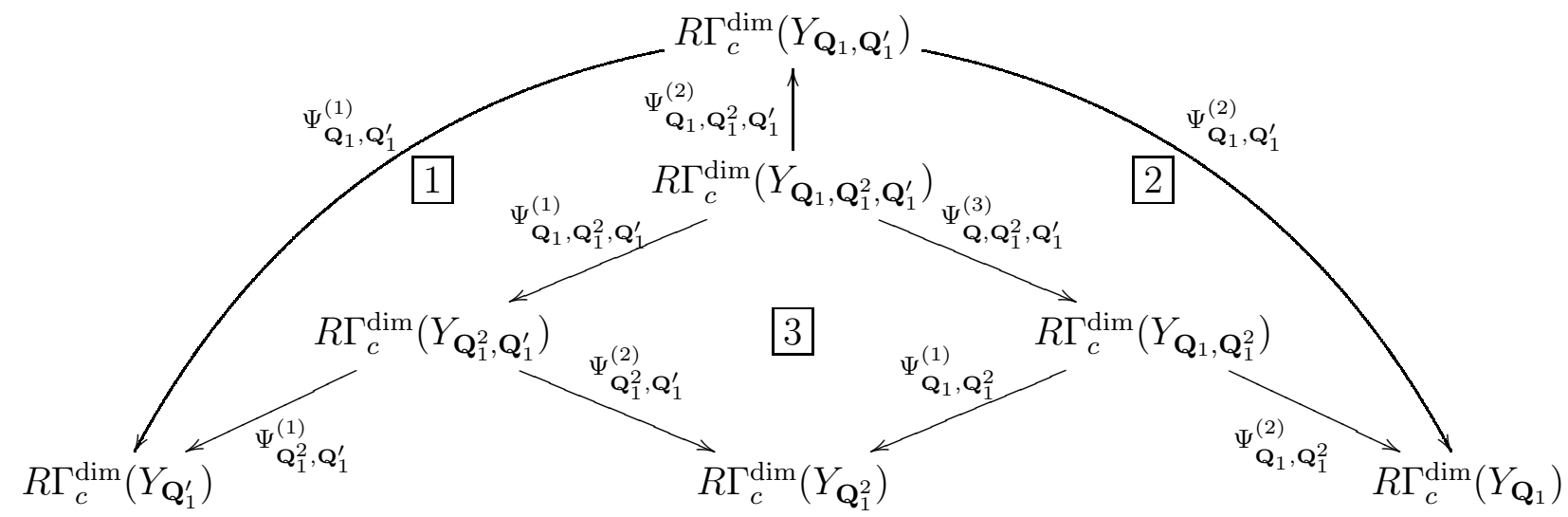

When we apply the idempotent $e_{s^{\prime}, \Lambda}^{M_{1}^{\prime}}$ to this diagram, all morphisms become isomorphisms (and all complexes are concentrated in degree 0$)$. Therefore, to prove (2.2.10), it is enough to prove the commutativity of subdiagrams 1,2 and 3 . Each of these diagrams is isomorphic to a diagram of the following form

$$
\begin{aligned}
& R \Gamma_{c}^{\operatorname{dim}}\left(Y_{\mathbf{R}_{1}, \mathbf{R}_{2}}\right) \stackrel{\Psi_{\mathbf{R}_{1}, \mathbf{R}_{2}, \mathbf{R}_{3}}^{(3)}}{\longrightarrow} R \Gamma_{c}^{\operatorname{dim}}\left(Y_{\mathbf{R}_{1}, \mathbf{R}_{2}, \mathbf{R}_{3}}\right)
\end{aligned}
$$

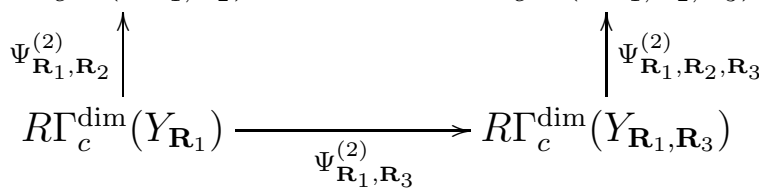

in which the morphisms $\Psi_{\mathbf{R}_{1}, \mathbf{R}_{2}, \mathbf{R}_{3}}^{(3)}$ and $\Psi_{\mathbf{R}_{1}, \mathbf{R}_{2}, \mathbf{R}_{3}}^{(2)}$ are defined in a way similar to what we explained above (2.1.8) for $\Psi_{\mathbf{R}_{1}, \mathbf{R}_{2}}^{(2)}$. We refer to section $6 . \mathrm{B}$ of [1] for more details (see also section 2 of [4]). In particular, Lemma 6.14 of [1] (or Corollary 2.9 of [4]) tells us that such a diagram is commutative provided one of the following inclusion holds.

a. $\mathbf{R}_{2} \subset \mathbf{R}_{1} \mathbf{R}_{\mathbf{3}}$, or b. $F\left(\mathbf{R}_{1}\right) \subset \mathbf{R}_{1} \mathbf{R}_{\mathbf{3}}$, or c. $\mathbf{R}_{3} \subset \mathbf{R}_{2} F\left(\mathbf{R}_{\mathbf{1}}\right)$, or d. $\mathbf{R}_{1} \subset \mathbf{R}_{2} F\left(\mathbf{R}_{\mathbf{1}}\right)$.

Diagram 2. Here we are in the situation $\mathbf{R}_{1}=\mathbf{Q}_{1}, \mathbf{R}_{2}=\mathbf{Q}_{1}^{2}$ and $\mathbf{R}_{3}=\mathbf{Q}_{1}^{\prime}$. From the definition of these parabolic subgroups, we see that

$$
\mathbf{Q}_{1}^{2}=\left(\mathbf{P}_{\iota} \cap \mathbf{P}_{1}\right)\left(\mathbf{U}_{\iota} \cap \mathbf{P}_{2}\right) \mathbf{U}_{2} \subset\left(\mathbf{P}_{\iota} \cap \mathbf{P}_{1}\right) \mathbf{U}_{\iota}\left(\mathbf{P}_{\iota} \cap \mathbf{P}_{1}\right) \mathbf{U}_{1}=\mathbf{Q}_{1} \mathbf{Q}_{1}^{\prime},
$$

so we are in case a. and we conclude that diagram 2 is commutative.

Diagram 1. Taking into account the roundabout definitions of morphisms $\Psi^{(1)}$ (through certain equivalences of étale sites), we are in the situation $\mathbf{R}_{1}=\mathbf{Q}_{1}^{\prime}, \mathbf{R}_{2}=F\left(\mathbf{Q}_{1}\right)$ and $\mathbf{R}_{3}=$ $F\left(\mathbf{Q}_{1}^{2}\right)$. As above, we have $F\left(\mathbf{Q}_{1}^{2}\right) \subset F\left(\mathbf{Q}_{1}\right) F\left(\mathbf{Q}_{1}^{\prime}\right)$, so we are in case c. and we conclude that diagram 1 is commutative.

Diagram 3. Here we are in the situation $\mathbf{R}_{1}=\mathbf{Q}_{1}^{2}, \mathbf{R}_{2}=\mathbf{Q}_{1}^{\prime}$ and $\mathbf{R}_{3}=F\left(\mathbf{Q}_{1}\right)$. We have

$$
F\left(\mathbf{Q}_{1}^{2}\right)=\left(F\left(\mathbf{P}_{\iota}\right) \cap \mathbf{P}_{1}\right)\left(F\left(\mathbf{U}_{\iota}\right) \cap \mathbf{P}_{2}\right) \mathbf{U}_{2} \subset F\left(\mathbf{Q}_{1}\right) \mathbf{U}_{2} \subset F\left(\mathbf{Q}_{1}\right) \mathbf{Q}_{1}^{2},
$$

so we are in case b. and we conclude that diagram 3 is commutative. 


\section{From level 0 blocks to coefficient systems}

We now denote by $F$ a local non-archimedean field with ring of integers $\mathcal{O}$ and residue field $\mathbb{F}$. We fix a vector space $V$ over $F$ of dimension $n$ and are interested in the smooth representation theory of the $p$-adic group $G=\operatorname{Aut}_{F}(V)$.

\subsection{Review of coefficients systems and systems of idempotents}

3.1.1 Coefficient systems on simplicial complexes. - Recall that a simplicial complex $X=\left(X, X_{\bullet}\right)$ is a pair of sets with $X_{\bullet}$ consisting of finite subsets of $X$, being stable under inclusion and containing all singletons. We'll denote by $X_{d} \subset X$. the set of $d$-simplices (of cardinality $d+1$ ) and identify $X$ to $X_{0}$ (the set of vertices). We also denote by $[X]$ the small category whose set of objects is $X_{\bullet}$ and morphisms are given by inclusion, i.e.

$$
\operatorname{Hom}_{[X]}(\sigma, \tau)=\left\{1_{\sigma \tau}\right\} \text { if } \sigma \supseteq \tau \text { and } \operatorname{Hom}_{[X]}(\sigma, \tau)=\emptyset \text { else. }
$$

A coefficient system on $X$ with values in a category $\mathcal{C}$ is a functor $\mathcal{E}:[X] \longrightarrow \mathcal{C}$. When $\mathcal{C}$ is an abelian category with arbitrary direct sums, we may form "the" chain complex of a coefficient system

$$
\mathcal{C}_{*}(\mathcal{E}): \cdots \longrightarrow \cdots \bigoplus_{\sigma \in X_{n-1}} \mathcal{E}_{\sigma} \longrightarrow \cdots \longrightarrow \bigoplus_{\sigma \in X_{0}} \mathcal{E}_{\sigma}
$$

once an orientation of $X$ has been chosen. The first homology object $H^{0}(\mathcal{E})$ is then canonically isomorphic to the colimit $\operatorname{colim}_{[X]} \mathcal{E}$ of the functor $\mathcal{E}$.

Suppose further that $X$ carries an action of an abstract group $G$ that respects the simplicial structure. We then denote by $[X / G]$ the small category whose set of objects is $X$ • and morphisms are given by the action of $G$, i.e.

$$
\operatorname{Hom}_{[X / G]}(\sigma, \tau)=G_{\sigma \tau}^{\dagger}:=\{g \in G, \tau \subseteq g \sigma\} .
$$

Hence $[X / G]$ contains $[X]$. Now, a $G$-equivariant coefficient system on $X$ with values in a category $\mathcal{C}$ is a functor $\mathcal{E}:[X / G] \longrightarrow \mathcal{C}$. For each $\sigma \in X_{\bullet}$, the object $\mathcal{E}_{\sigma}$ then gets an action of the stabilizer $G_{\sigma}^{\dagger}$ of $\sigma$ and the colimit $\operatorname{colim}_{[X]} \mathcal{E}$ gets an action of $G$, as well as the complex $\mathcal{C}_{*}(\mathcal{E})$ when $\mathcal{C}$ is abelian. If $G$ is a topological group and acts continously on $X$ with the discrete topology, we say that $\mathcal{E}$ is smooth if for each $\sigma \in X_{\bullet}$, the action of $G_{\sigma}^{\dagger}$ on $\mathcal{E}_{\sigma}$ is so. Then the action of $G$ on $\operatorname{colim}_{[X]} \mathcal{E}$ and $\mathcal{C}_{*}(\mathcal{E})$ is also smooth.

We will generally denote by $G_{\sigma}$ the pointwise stabilizer of $\sigma$, which is a distinguished subgroup of $G_{\sigma}^{\dagger}$. For $\tau \subseteq \sigma$ we have $G_{\sigma} \subset G_{\tau}$, but in general $G_{\sigma}^{\dagger}$ is not contained in $G_{\tau}^{\dagger}$.

3.1.2 The Bruhat-Tits building. - Here we take up our notation $G=\operatorname{Aut}_{F}(V)$ of the beginning of this section. We introduce the semi-simple building $B T$ associated to $G$, which we view as a simplicial complex of dimension $n-1$ with a simplicial action of $G$.

Denote by $\operatorname{Latt}(V)$ the set of $\mathcal{O}_{F}$-lattices in $V$. A lattice chain is a decreasing sequence $\mathbb{Z} \longrightarrow \operatorname{Latt}(V), i \mapsto \mathcal{L}_{i}$. It is called $d+1$-periodic if $\mathcal{L}_{i+d}=\varpi \mathcal{L}_{i}$ for all $i$. Moreover, two such sequences are called equivalent if one is a shift of the other one. Denote by $B T_{d}$ the set of equivalence classes of $d$-periodic lattice chains. It turns out that such an equivalence class is completely determined by the $d+1$ equivalence classes of 1-periodic lattice chains that can be 
extracted from it. Whence an embedding of $B T_{d}$ in the power set of $B T:=B T_{0}$, that is part of a simplicial complex structure $\left(B T, B T_{\bullet}\right)$, for which a $d^{\prime}$-simplex $\tau$ is a facet of a $d$-simplex $\sigma$ if a lattice chain in $\tau$ is extracted from one in $\sigma$.

The action of $G$ on $\operatorname{Latt}(V)$ induces a simplicial and continuous action on $B T$. Let $G^{0}:=$ $\operatorname{ker}(|\operatorname{det}|) \subset G$. The group $G_{\sigma}^{0}:=G_{\sigma}^{\dagger} \cap G^{0}$ is the maximal compact subgroup of the stabilizer $G_{\sigma}^{\dagger}$ of a simplex $\sigma$. It is a normal subgroup and we have $G_{\sigma}=G_{\sigma}^{0} Z$ with $Z$ denoting the center of $G$, while $G_{\sigma}^{\dagger} / G_{\sigma}$ is a cyclic group of order dividing $n$.

3.1.3 Consistent systems of idempotents and Serre subcategories. - Let $R$ be a commutative ring in which $p$ is invertible and let $\mathcal{H}_{R}(G)$ denote the algebra of locally constant $R$-valued compactly supported measures on $G$. We are interested in collections of idempotents $\left(e_{x}\right)_{x \in B T_{0}}$ in $\mathcal{H}_{R}(G)$ that satisfy the following consistency properties, taken from [8, $\left.\S 2.1\right]$.

i) $e_{x} e_{y}=e_{y} e_{x}$ whenever $x$ and $y$ are adjacent.

ii) $e_{x} e_{z} e_{y}=e_{x} e_{y}$ whenever $z$ belongs the simplicial hull (enclos) of $\{x, y\}$.

iii) $e_{g x}=g e_{x} g^{-1}$ for all $x$ and all $g \in G$.

Note that condition i) enables one to define an idempotent $e_{\sigma}=\prod_{x \leqslant \sigma} e_{x}$ for each simplex $\sigma$. Moreover we have $e_{\sigma} e_{\tau}=e_{\sigma}$ for $\tau \subseteq \sigma$ and $e_{g \sigma}=g e_{\sigma} g^{-1}$ for $g \in G$. Note also that the whole system is determined by a single $e_{x}$, since in our $\mathrm{GL}_{n}$ setting, all vertices are conjugated.

Our interest in this notion comes from the following result of Meyer and Solleveld. Recall that $\operatorname{Rep}_{R}(G)$ is the category of smooth $R$-representations of $G$.

Fact. ([8], Thm 3.1) - Let $e=\left(e_{x}\right)_{x \in B T}$ be a consistent system of idempotents as above. Then the full subcategory $\operatorname{Rep}_{R}^{e}(G)$ of all objects $V$ in $\operatorname{Rep}_{R}(G)$ such that $V=\sum_{x} e_{x} V$ is a Serre sub-category.

In fact, level decompositions on $\operatorname{Rep}_{R}(G)$ show that $\operatorname{Rep}_{R}^{e}(G)$ is a direct factor subcategory, or in other words, that $\mathcal{H}_{R}(G) e_{x} \mathcal{H}_{R}(G)$ is a direct factor two-sided ideal of $\mathcal{H}_{R}(G)$.

3.1.4 Resolutions and equivalences of categories. - The most important ingredient in the proof of the above Fact is a certain acyclicity result. Namely, for a representation $V$, define the coefficient system $\left(\mathcal{V}_{\sigma}\right)_{\sigma \in B T}$ by putting $\mathcal{V}_{\sigma}:=e_{\sigma} V$ for all $\sigma \in B T$. and $\mathcal{V}\left(g_{\sigma \tau}\right): e_{\sigma} V \longrightarrow e_{\tau} V$ the map induced by the action of $g$ on $V$, for all $g \in G_{\sigma \tau}^{\dagger}$ (recall that $e_{\tau} e_{g \sigma}=e_{g \sigma}$ ). Then the complex $\mathcal{C}_{*}(\mathcal{V})$ has a natural augmentation given by $\bigoplus_{x \in B T_{0}} e_{x} V \stackrel{\Sigma}{\longrightarrow} V$.

Fact. ([8], Thm 2.4) — The complex $\mathcal{C}_{*}(\mathcal{V})$ is acyclic in positive degrees and the augmentation map is an isomorphism $H_{0}\left(\mathcal{C}_{*}(\mathcal{V})\right) \stackrel{\sim}{\longrightarrow} \sum_{x} e_{x} V$.

Denote by Coef $_{R}[B T / G]$ the category of $G$-equivariant coefficient systems in $R$-modules on the building $B T$. The above result suggests one to consider the full subcategory of all coefficient systems $\mathcal{V}$ such that $\mathcal{V}\left(1_{\sigma \tau}\right)$ induces an isomorphism $\mathcal{V}_{\sigma} \stackrel{\sim}{\longrightarrow} e_{\sigma} \mathcal{V}_{\tau}$ for all $\tau \subseteq \sigma$ (equivalently, $\mathcal{V}\left(g_{\sigma \tau}\right)$ induces an isomorphism $\mathcal{V}_{\sigma} \stackrel{\sim}{\longrightarrow} e_{g \sigma} \mathcal{V}_{\tau}$ for all $\left.g \in G_{\sigma \tau}^{\dagger}\right)$. In order to be able to do this, one needs to impose a further axiom on the system $e$, namely

iv) for any $\sigma, e_{\sigma}$ is supported in $G_{\sigma}$. 
Indeed, under this assumption, since $G_{\sigma} \subset G_{\tau}$ for $\tau \subseteq \sigma$ and since $\mathcal{V}_{\tau}$ comes with a smooth action of $G_{\tau}$, we can unambiguously make sense of the expression $e_{\sigma} \mathcal{V}_{\tau}$. Denote by $\operatorname{Coef}_{R}^{e}[B T / G]$ the category of these coefficients systems. Wang has extended the above fact in the following way.

3.1.5 Fact ([10], Thm 2.1.9 and Cor. 2.1.10) - For $\mathcal{V} \in \operatorname{Coef}_{R}^{e}[B T / G]$ the complex $\mathcal{C}_{*}(\mathcal{V})$ is acyclic in positive degrees. Moreover the functor $\mathcal{V} \mapsto H_{0}\left(\mathcal{C}_{*}(\mathcal{V})\right)$ provides an equivalence of categories

$$
\operatorname{Coef}_{R}^{e}[B T / G] \stackrel{\sim}{\longrightarrow} \operatorname{Rep}_{R}^{e}(G)
$$

with quasi-inverse the functor $V \mapsto\left(e_{\sigma} V\right)_{\sigma}$.

We note that Meyer and Solleveld results are valid for any reductive group over $F$, while Wang's result has been written only for $\mathrm{GL}_{n}$.

3.1.6 The level 0 example. - For a vertex $x$, denote by $G_{x}^{+}$the kernel of the action map $G_{x}^{0} \longrightarrow \operatorname{Aut}_{\mathbb{F}}\left(\mathcal{L} \otimes_{\mathcal{O}} \mathbb{F}\right)$. It is independent of the choice of $\mathcal{L}$ and coincides with the pro-p-radical of $G_{x}$. In particular it determines an idempotent $e_{x}^{+} \in \mathcal{H}_{\mathbb{Z}[1 / p]}\left(G_{x}\right)$.

The system $\left(e_{x}^{+}\right)_{x \in B T_{0}}$ is well known to be consistent in the above sense. Moreover, for any simplex $\sigma$, the idempotent $e_{\sigma}^{+}:=\prod_{x \in \sigma} e_{x}^{+}$is nothing but the idempotent associated to the pro- $p$ radical $G_{\sigma}^{+}$of $G_{\sigma}^{0}$, the latter being also the kernel of the natural map $G_{\sigma}^{0} \longrightarrow \prod_{i} \operatorname{Aut}_{\mathbb{F}}\left(\mathcal{L}_{i} / \mathcal{L}_{i+1}\right)$ whenever $\sigma$ is represented by the lattice chain $\left(\mathcal{L}_{i}\right)_{i}$. In particular, property iv) above is also satisfied.

We denote by $\operatorname{Rep}_{R}^{0}(G)$ the direct factor of $\operatorname{Rep}_{R}(G)$ cut out by the system $\left(e_{x}^{+}\right)_{x}$. It is usually called the level 0 subcategory. Similarly we write $\operatorname{Coef}_{R}^{0}[B T / G]$ for the corresponding category of equivariant coefficient systems.

\subsection{Level 0 blocks}

Let $\Lambda$ denote either $\overline{\mathbb{Q}}_{\ell}$ or $\overline{\mathbb{Z}}_{\ell}$ (or $\overline{\mathbb{F}}_{\ell}$ ). Fix a semisimple conjugacy class $s$ in $\mathrm{GL}_{n}(\mathbb{F})$ and assume it has order invertible in $\Lambda$. Since $\mathrm{GL}_{n}$ is its own dual, paragraph 2.1 .3 provides us with a central idempotent $e_{s, \Lambda}^{\mathrm{GL}_{n}(\mathbb{F})}$ in $\Lambda\left[\mathrm{GL}_{n}(\mathbb{F})\right]$.

3.2.1 The systems of idempotents attached to $s$. - Let $x$ be a vertex. Let us choose a lattice $\mathcal{L}$ corresponding to $x$ and an $\mathcal{O}$-basis of $\mathcal{L}$. Then we get an isomorphism

$$
\bar{G}_{x}:=G_{x} / G_{x}^{+} \stackrel{\sim}{\longrightarrow} \operatorname{Aut}_{\mathbb{F}}\left(\mathcal{L} \otimes_{\mathcal{O}} \mathbb{F}\right) \stackrel{\sim}{\longrightarrow} \operatorname{GL}_{n}(\mathbb{F})
$$

which allows us to pull back $e_{s, \Lambda}^{\mathrm{GL}_{n}(\mathbb{F})}$ to a central idempotent $e_{x}^{s, \Lambda} \in \Lambda\left[\bar{G}_{x}\right] \subset \mathcal{H}_{\Lambda}\left(G_{x}\right)$. Since it is central it is in fact independent of the choices of $\mathcal{L}$ and the basis.

Lemma. - The system $\left(e_{x}^{s, \Lambda}\right)_{x \in B T_{0}}$ is consistent in the sense of 3.1 .3 and satisfies the additional property iv) of 3.1.4. Moreover, for any two vertices $x, y$ we have

$$
e_{x}^{+} e_{y}^{s, \Lambda}=e_{x}^{s, \Lambda} e_{y}^{s}
$$

Proof. We will first extend the system to all simplices. Let $\sigma$ be a simplex represented by a periodic lattice chain $\cdots \supset \mathcal{L}_{0} \supset \mathcal{L}_{1} \supset \cdots$. We thus get a partial flag in the vector space 
$\mathcal{L}_{0} \otimes_{\mathcal{O}} \mathbb{F}$. After choosing an $\mathcal{O}$-basis of $\mathcal{L}_{0}$ we get a parabolic subgroup $P_{\sigma}$ of $\mathrm{GL}_{n}(\mathbb{F})$ and a surjection $G_{\sigma}^{0} \rightarrow P_{\sigma}$. Let $U_{\sigma}$ be the radical of $P_{\sigma}$ and $e_{U_{\sigma}}$ the corresponding idempotent. Then by (2.1.5) the idempotent $e_{s, \Lambda}^{\mathrm{GL}_{n}(\mathbb{F})} e_{U_{\sigma}}$ is a central idempotent in $\Lambda\left[P_{\sigma}\right]$, and therefore defines a central idempotent $e_{\sigma}^{s, \Lambda} \in \mathcal{H}_{\Lambda}\left(G_{\sigma}\right)$. Note that changing the lattice chain or the $\mathcal{O}$-basis will result in conjugating the parabolic subgroup $P_{\sigma}$ in $\mathrm{GL}_{n}(\mathbb{F})$. Therefore $e_{\sigma}^{s, \Lambda}$ is independent of these choices. Moreover, by construction, we have

$$
e_{\sigma}^{s, \Lambda}=e_{\sigma}^{+} e_{x}^{s, \Lambda} \text { for any vertex } x \leqslant \sigma,
$$

and where $e_{\sigma}^{+}$is the idempotent associated to $G_{\sigma}^{+}$as in 3.1.6.

We now can prove that these systems are consistent. For the sake of readability, we omit the superscript $\Lambda$. For property i) of 3.1.3, let $x, y$ be adjacent vertices and $\sigma$ the simplex $\{x, y\}$. Then we know that $e_{x}^{+} e_{y}^{+}=e_{\sigma}^{+}$. Therefore we get

$$
e_{x}^{s} e_{y}^{s}=e_{x}^{s} e_{x}^{+} e_{y}^{+} e_{y}^{s}=e_{x}^{s} e_{\sigma}^{+} e_{\sigma}^{+} e_{y}^{s}=e_{\sigma}^{s} e_{\sigma}^{s}=e_{\sigma}^{s} .
$$

This proves property i). Moreover, we see in the same way that $e_{\sigma}^{s}=\prod_{x \leqslant \sigma} e_{x}^{s}$ for any simplex $\sigma$, so that property iv) of 3.1 .4 also holds. Since property iii) is clear, it only remains to check property ii). So let $z$ belong to the enclos of $\{x, y\}$. We already know that $e_{x}^{+} e_{y}^{+}=e_{x}^{+} e_{z}^{+} e_{y}^{+}$. This implies the desired property when $z$ is adjacent to $x$, since in this case we have

$$
e_{x}^{s} e_{y}^{s}=e_{x}^{s} e_{z}^{+} e_{y}^{s}=e_{x}^{s} e_{[x, z]}^{+} e_{y}^{s}=e_{[x, z]}^{s} e_{y}^{s}=e_{x}^{s} e_{z}^{s} e_{y}^{s} .
$$

When $z$ is not adjacent to $x$, we can find a path $x=x_{0}, x_{1}, \cdots, x_{r}=z, \cdots, x_{l}=y$ such that $x_{i+1}$ is adjacent to $x_{i}$ and belongs to the enclos of $\left\{x_{i}, y\right\}$ for each $i$, as in [10, Lemme (2.2.5)]. Then by induction we get property ii).

Finally we prove equality (3.2.2). When $x$ and $y$ are adjacent we already know that

$$
e_{x}^{+} e_{y}^{s}=e_{[x, y]}^{+} e_{y}^{s}=e_{[x, y]}^{s}=e_{x}^{s} e_{y}^{s}
$$

as desired. In general, we choose a path $x=x_{0}, \cdots x_{l}=y$ as above. Since $x_{l-1}$ is in the enclos of $\{x, y\}$ and is adjacent to $y$, we have

$$
e_{x}^{+} e_{y}^{s}=e_{x}^{+} e_{x_{l-1}}^{+} e_{y}^{s}=e_{x}^{+} e_{x_{l-1}}^{s} e_{y}^{s} .
$$

By induction on $l$, we deduce that

$$
e_{x}^{+} e_{y}^{s}=e_{x}^{s} e_{x_{1}}^{s} \cdots e_{x_{l-1}}^{s} e_{y}^{s}=e_{x}^{s} e_{y}^{s}
$$

as claimed.

We will denote by $\operatorname{Rep}_{\Lambda}^{s}(G)$ the Serre subcategory of $\operatorname{Rep}_{\Lambda}(G)$ cut out by the system of idempotents $\left(e_{x}^{s, \Lambda}\right)_{x}$. It is pro-generated by the induced object $\operatorname{ind}_{G_{x}^{0}}^{G}\left(e_{x}^{s, \Lambda} \mathcal{H}_{\Lambda}\left(G_{x}\right)\right)$ for any

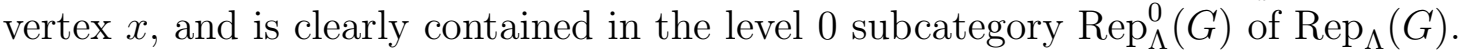

3.2.4 Proposition. - The level 0 subcategory $\operatorname{Rep}_{\Lambda}^{0}(G)$ splits as a direct product

$$
\operatorname{Rep}_{\Lambda}^{0}(G)=\prod_{s \in \mathrm{GL}_{n}(\mathbb{F})^{\mathrm{ss}, \Lambda} / \mathrm{conj}} \operatorname{Rep}_{\Lambda}^{s}(G)
$$

where $s$ runs over semisimple conjugacy classes in $\mathrm{GL}_{n}(\mathbb{F})$ which have invertible order in $\Lambda$. 
Proof. Let $s, s^{\prime}$ be two distinct conjugacy classes with invertible order in $\Lambda$. For simplicity we simply write $e_{x}^{s}=e_{x}^{s, \Lambda}$ and $e_{x}^{s^{\prime}}=e_{x}^{s^{\prime}, \Lambda}$. We first show that $\operatorname{Rep}_{\Lambda}^{s}(G)$ and $\operatorname{Rep}_{\Lambda}^{s^{\prime}}(G)$ are orthogonal. Indeed, let $V$ be an object in $\operatorname{Rep}_{\Lambda}^{s}(G)$. We have to prove that $e_{x}^{s^{\prime}} V=0$ for any vertex $x$. We know that $V=\sum_{y} e_{y}^{s} V$, so that $e_{x}^{s^{\prime}} V=\sum_{y} e_{x}^{s^{\prime}} e_{y}^{s} V$. But for all $x, y$, using (3.2.2) we get

$$
e_{x}^{s^{\prime}} e_{y}^{s}=e_{x}^{s^{\prime}} e_{x}^{+} e_{y}^{s}=e_{x}^{s^{\prime}} e_{x}^{s} e_{y}^{s}=0
$$

since $e_{x}^{s^{\prime}} e_{x}^{s}=0$

Now, let $V$ be a level 0 object that is orthogonal to all subcategories $\operatorname{Rep}_{\Lambda}^{s}(G)$. For a fixed vertex $x$, we thus have $e_{x}^{s}$ for all $s$. But by $\left.2.1 .3 \mathrm{i}\right)$ we know that $e_{x}^{+}=\sum_{s} e_{x}^{s}$, so $e_{x}^{+} V=0$ hence $V=0$.

This finishes the proof of the claimed decomposition. Note that, concretely, the projection of a level 0 object $V$ on $\operatorname{Rep}_{\Lambda}^{s}(G)$ is canonically isomorphic to the subrepresentation generated by $e_{x}^{s, \Lambda} V$.

The link between the decompositions over $\overline{\mathbb{Z}}_{\ell}$ and $\overline{\mathbb{Q}}_{\ell}$ is quite clear : for an $\ell$-regular semisimple class $s$ we have

$$
\operatorname{Rep}_{\overline{\mathbb{Z}}_{\ell}}^{s}(G) \cap \operatorname{Rep}_{\overline{\mathbb{Q}}_{\ell}}(G)=\prod_{s^{\prime} \sim \sim_{\ell}} \operatorname{Rep}_{\mathbb{Q}_{\ell}}^{s^{\prime}}(G)
$$

3.2.5 Relation to Bernstein blocks. - Here we consider the case $\Lambda=\overline{\mathbb{Q}}_{\ell}$. Fix a conjugacy class $s$ as above. There is unique partition $\left(n_{1} \geqslant n_{2} \geqslant \cdots \geqslant n_{r}\right)$ of $n$ such that $s$ contains an elliptic element of the diagonal Levi subgroup $M(\mathbb{F})=\mathrm{GL}_{n_{1}}(\mathbb{F}) \times \cdots \times \mathrm{GL}_{n_{r}}(\mathbb{F})$. As in $2.1 .10 \mathrm{i}$ ), we thus get from $s$ a supercuspidal representation $\bar{\sigma}_{1} \otimes \cdots \otimes \bar{\sigma}_{r}$ of this Levi subgroup. After inflating to $M(\mathcal{O})=\prod_{i} \mathrm{GL}_{n_{i}}(\mathcal{O})$, extending to $\prod_{i} F^{\times} \mathrm{GL}_{n_{i}}(\mathcal{O})$, and inducing to $M(F)=\mathrm{GL}_{n_{1}}(F) \times \cdots \times \mathrm{GL}_{n_{r}}(F)$, we get a supercuspidal representation $\sigma=\sigma_{1} \otimes \cdots \otimes \sigma_{r}$ of the $p$-adic group $M(F)$. Choosing further an $F$-basis of $V$ we get a cuspidal pair $(M, \sigma)$ in $G$ whose inertial class does not depend on any choice (basis and extension). In view of the well known construction of level 0 supercuspidal representations, this process sets up a bijection

\{semisimple conj. classes in $\left.\mathrm{GL}_{n}(\mathbb{F})\right\} \leftrightarrow\{$ inertial classes of level 0 supercuspidal pairs in $G$ \}

Now, attached to $[M, \sigma]$ is the Bernstein block $\operatorname{Rep}_{\overline{\mathbb{Q}}_{\ell}}^{[M, \sigma]}(G)$ of $\operatorname{Rep}_{\overline{\mathbb{Q}}_{\ell}}(G)$ which consists of all objects, all irreducible subquotients of which have supercuspidal support inertially equivalent to $[M, \sigma]$. Bernstein's decomposition, when restricted to the level 0 subcategory, then reads

$$
\operatorname{Rep}_{\overline{\mathbb{Q}}_{\ell}}^{0}(G)=\prod_{[M, \sigma]} \operatorname{Rep}_{\overline{\mathbb{Q}}_{\ell}}^{[M, \sigma]}(G)
$$

Proposition. - We have $\operatorname{Rep}_{\mathbb{Q}_{\ell}}^{s}(G)=\operatorname{Rep}_{\overline{\mathbb{Q}}_{\ell}}^{[M, \sigma]}(G)$. In particular $\operatorname{Rep}_{\mathbb{Q}_{\ell}}^{s}(G)$ is a block.

Proof. In view of the two above decompositions of $\operatorname{Rep}_{\overline{\mathbb{Q}}_{\ell}}^{0}(G)$ and the bijection $s \leftrightarrow[M, \sigma]$, it will be sufficient to prove the inclusion $\operatorname{Rep}_{\overline{\mathbb{Q}}_{\ell}}^{s}(G) \supset \operatorname{Rep}_{\overline{\mathbb{Q}}_{\ell}}^{[M, \sigma]}(G)$ for $s$ corresponding to $[M, \sigma]$.

For this it suffices to prove that any irreducible $\pi$ which occurs as a subquotient of an induced representation $i_{P}^{G}\left(\sigma_{1} \otimes \cdots \otimes \sigma_{r}\right)$ as above (where $P$ is a parabolic subgroup with Levi $M)$ satisifies $e_{x}^{s} \pi \neq 0$ for some vertex $x$. 
For simplicity, let us choose an $F$ basis of $V$ such that the identification $G=\operatorname{GL}_{n}(F)$ takes $M$ to the diagonal Levi subgroup $\prod_{i} \mathrm{GL}_{n_{i}}(F)$ and $P$ to the corresponding upper-triangular group. Then consider the vertex $x$ associated to the lattice spanned by this basis, so that $G_{x}^{0}=\mathrm{GL}_{n}(\mathcal{O})$. Finally denote by $\bar{P}_{x}$ the image of $P \cap G_{x}^{0}$ in $\bar{G}_{x}$. Then the Mackey formula shows that, as a representation of $\bar{G}_{x}$ we have

$$
i_{P}^{G}\left(\sigma_{1} \otimes \cdots \otimes \sigma_{r}\right)^{G_{x}^{+}} \simeq i_{\bar{P}_{x}}^{\bar{G}_{x}}\left(\bar{\sigma}_{1} \otimes \cdots \otimes \bar{\sigma}_{r}\right) .
$$

where $i_{P}^{G}$ denotes parabolic induction. Therefore $\pi^{G_{x}^{+}}$is a subquotient of $i_{\bar{P}_{x}}^{\bar{G}_{x}}\left(\bar{\sigma}_{1} \otimes \cdots \otimes \bar{\sigma}_{r}\right)$ and by 2.1 .10 ii) we get that $e_{x}^{s} \pi \neq 0$.

3.2.6 Compatibility with Langlands' correspondence. — As in 1.1.2, the local Langlands correspondence gives a parametrization of blocks of $\operatorname{Rep}_{\overline{\mathbb{Q}}_{\ell}}\left(\mathrm{GL}_{n}(F)\right)$ by the set $\Phi_{\text {inert }}\left(\mathrm{GL}_{n}, \overline{\mathbb{Q}}_{\ell}\right)$ of equivalence classes of semi-simple representations of $I_{F}$ that extend to $W_{F}$. Moreover, level 0 blocks correspond to tame parameters through this parametrization. Sofar, we have defined three maps :

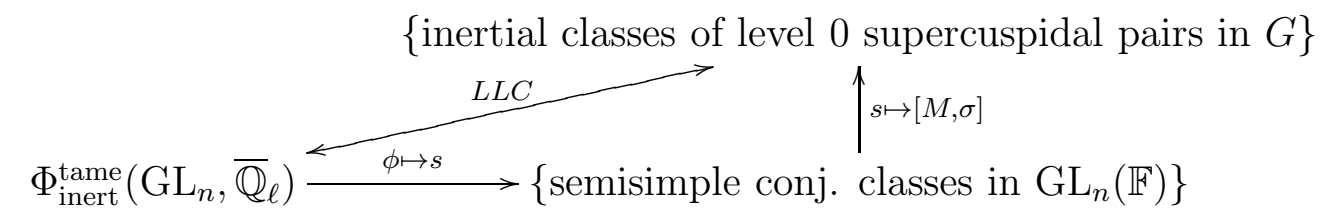

The vertical map was defined in 3.2.5, while the horizontal one was defined in 1.2.1.

Proposition. - This diagram commutes. In particular we have $\operatorname{Rep}_{\phi}(G)=\operatorname{Rep}_{\mathbb{Q}_{\ell}}(G)$.

Proof. Let us start with a semisimple element $s \in \mathrm{GL}_{n}(\mathbb{F})$, let us denote by $[M, \sigma]$ the corresponding supercuspidal pair, by $\phi$ the inertial parameter of $[M, \sigma]$, and by $s(\phi)$ the conjugacy class associated to $\phi$. We want to prove that $s \in s(\phi)$.

We first reduce to the case where $s$ is elliptic. Indeed, after maybe conjugating we may assume that $s=s_{1} \times \cdots \times s_{r}$ is elliptic in the Levi subgroup $M(\mathbb{F})=\mathrm{GL}_{n_{1}}(\mathbb{F}) \times \cdots \times \mathrm{GL}_{n_{r}}(\mathbb{F})$. Then we have $M=\mathrm{GL}_{n_{1}} \times \cdots \times \mathrm{GL}_{n_{r}}$ and $\sigma=\sigma_{1} \otimes \cdots \otimes \sigma_{r}$ with each $\sigma_{i}$ corresponding to $s_{i}$. Next, putting $\phi_{i}:=\left(\varphi_{\sigma_{i}}\right)_{\mid I_{F}}$, we have $\phi=\phi_{1} \oplus \cdots \oplus \phi_{r}$, and the conjugacy class $s(\phi)$ is by definition that of a product $s\left(\phi_{1}\right) \times \cdots \times s\left(\phi_{r}\right)$ in $M(\mathbb{F}) \subset \mathrm{GL}_{n}(\mathbb{F})$. Therefore we are reduced to check $s_{i} \in s\left(\phi_{i}\right)$ for each $i$.

Now assume $s$ is elliptic, so that $M=G$. This case boils down to the well-known compatibility between the local Langlands correspondence and the Green-Deligne-Lusztig construction (up to some unramified rectifying character).

3.2.7 Relation to Vignéras-Helm blocks. - Here we consider the case $\Lambda=\overline{\mathbb{Z}}_{\ell}$ and we suppose that $s$ is an $\ell$-regular semisimple conjugacy class of $\mathrm{GL}_{n}(\mathbb{F})$. Using the notation of paragraph 3.2 .5 , we now get from $s$ a supercuspidal $\overline{\mathbb{F}}_{\ell}$-representation of $M(\mathbb{F})$, thanks to 2.1.10 iii). Again, after inflating, extending, and inducing, we get an inertial class $[M, \sigma]$ of supercuspidal pairs over $\overline{\mathbb{F}}_{\ell}$ in $G$, and this process induces a bijection

$\left\{\ell\right.$-regular semisimple conj. classes in $\left.\mathrm{GL}_{n}(\mathbb{F})\right\}$ $\leftrightarrow$ inertial classes of level $0 \overline{\mathbb{F}}_{\ell^{-}}$-supercuspidal pairs in $\left.G\right\}$ 
Now, attached to $[M, \sigma]$ is the Vignéras-Helm block $\operatorname{Rep}_{\overline{\mathbb{Z}}_{\ell}}^{[M, \sigma]}(G)$ of $\operatorname{Rep}_{\overline{\mathbb{Z}}_{\ell}}(G)$ which consists of all objects, all simple subquotients of which have "mod $\ell$ inertial supercuspidal support" equal to $[M, \sigma]$. The level 0 subcategory then decomposes as

$$
\operatorname{Rep}_{\overline{\mathbb{Z}}_{\ell}}^{0}(G)=\prod_{[M, \sigma]} \operatorname{Rep}_{\overline{\mathbb{Z}}_{\ell}}^{[M, \sigma]}(G)
$$

the product being over $[M, \sigma]$ 's with level 0 .

Proposition. - We have $\operatorname{Rep}_{\overline{\mathbb{Z}}_{\ell}}^{s}(G)=\operatorname{Rep}_{\overline{\mathbb{Z}}_{\ell}}^{[M, \sigma]}(G)$. In particular $\operatorname{Rep}_{\overline{\mathbb{Z}}_{\ell}}^{s}(G)$ is a block.

Proof. As in the proof of Proposition 3.2.5, it suffices to prove the inclusion $\operatorname{Rep}_{\mathbb{Z}_{\ell}}(G) \supset$ $\operatorname{Rep}_{\overline{\mathbb{Z}}_{\ell}}^{[M, \sigma]}(G)$. On the other hand, since the LHS is a Serre subcategory and the RHS is a block, it suffices to find one object in the RHS that belongs to the LHS. Therefore it suffices to prove that any irreducible $\overline{\mathbb{F}}_{\ell^{-}}$-representation $\pi$ in $\operatorname{Rep}_{\overline{\mathbb{F}}_{\ell}}^{[M, \sigma]}(G)$ satisfies $e_{x}^{s, \overline{\mathbb{Z}}_{\ell}} \pi \neq 0$. By definition $\pi$ occurs as a subquotient of some $i_{P}^{G}\left(\sigma_{1} \otimes \cdots \otimes \sigma_{r}\right)$, so we may argue exactly as in the previous proposition, using 2.1.10 iv) instead of ii).

Let now $\phi \in \Phi_{\ell \text {-inert }}\left(\mathrm{GL}_{n}, \overline{\mathbb{Q}}_{\ell}\right)$ be the $\ell^{\prime}$-inertia parameter which corresponds to $[M, \sigma]$ as in 1.1.2, and let $s(\phi)$ be the conjugacy class associated to $\phi$ as in 1.2.1.

Proposition. - We have $s=s(\phi)$. In particular, we have $\operatorname{Rep}_{\mathbb{Z}_{\ell}}^{s}(G)=\operatorname{Rep}_{\phi}(G)$.

Proof. As in the proof of Proposition 3.2.6, we reduce to the case where $s$ is elliptic. Then we have $M=G$ and $\sigma$ is the reduction $\bmod \ell$ of the supercuspidal $\overline{\mathbb{Q}}_{\ell}$-representation $\widetilde{\sigma}$ attached to $s$. Moreover, by compatibility between Langlands and Vignéras correspondences for supercuspidal representations with respect to reduction $\bmod \ell$, the parameter $\phi$ is the restriction to $I_{F}^{\ell}$ of the inertial parameter $\widetilde{\phi}$ associated with $\widetilde{\sigma}$. Proposition 3.2 .6 tells us that $s(\widetilde{\phi})=s$, and 1.2 .1 tells us that $s(\phi)$ is the $\ell^{\prime}$-part of $s$, which is $s$ by assumption.

\section{From Coefficient systems to Hecke "modules"}

We take up the setting of the last section. So $G=\operatorname{Aut}_{F}(V)$ acts on its building $B T$, and $\Lambda=\overline{\mathbb{Z}}_{\ell}$ or $\overline{\mathbb{Q}}_{\ell}$. To any semisimple conjugacy class $s \in \mathrm{GL}_{n}(\mathbb{F})$ with order invertible in $\Lambda$, we have associated a system of idempotents $\left(e_{x}^{s, \Lambda}\right)_{x \in B T}$, heading to a block $\operatorname{Rep}_{\Lambda}^{s}(G)$ that is equivalent to a certain category $\operatorname{Coef}_{\mathbb{Z}_{\ell}}^{s}[B T / G]$ of equivariant coefficient systems on $B T$.

In this section we show how $\operatorname{Coef}_{\Lambda}^{s}[B T / G]$ is equivalent to a category of "modules" over a certain Hecke " $\Lambda$-algebra", whose definition only involves a single appartment $A$ and the collections of reductive quotients $\bar{G}_{\sigma}, \sigma \in A_{\text {• }}$. In this way, we obtain objects that are closer to the usual modules over Hecke algebras, except that our Hecke "algebras" will actually be "rings with many objects".

\subsection{The enriched Coxeter complex}

4.1.1 Level 0 coefficient systems. - Let $\mathcal{C}$ be any category. We say that a $\mathcal{C}$-valued $G$ equivariant coefficient system $\mathcal{E}:[B T / G] \longrightarrow \mathcal{C}$ has level 0 if for all $\sigma \in B T_{\bullet}$, the action of $G_{\sigma}^{+}$ 
on $\mathcal{E}_{\sigma}$ is trivial. Let us introduce a new category $[B T / \bar{G}]$ whose set of objects is still $B T_{\bullet}$, and morphisms are given by

$$
\bar{G}_{\sigma \tau}^{\dagger}:=G_{\tau}^{+} \backslash G_{\sigma \tau}^{\dagger} / G_{\sigma}^{+}=G_{\sigma \tau}^{\dagger} / G_{\sigma}^{+} .
$$

Note that the composition maps $G_{\sigma \tau}^{\dagger} \times G_{\tau \nu}^{\dagger} \longrightarrow G_{\sigma \nu}^{\dagger}$ induce associative composition maps on quotients $\bar{G}_{\sigma \tau}^{\dagger} \times \bar{G}_{\tau \nu}^{\dagger} \longrightarrow \bar{G}_{\sigma \nu}^{\dagger}$ so we indeed get a category $[B T / \bar{G}]$, which is a quotient category of $[B T / G]$. It is then clear that a $G$-equivariant coefficient system $\mathcal{E}$ has level 0 if and only if it factors through a functor $\mathcal{E}:[B T / \bar{G}] \longrightarrow \mathcal{C}$.

Definition. - We say that $\mathcal{E}:[B T / \bar{G}] \longrightarrow \mathcal{C}$ is cartesian if the transition map $\mathcal{E}\left(\overline{1}_{\sigma \tau}\right)$ is an isomorphism $\mathcal{E}_{\sigma} \stackrel{\sim}{\longrightarrow} \mathcal{E}_{\tau}^{G_{\sigma}^{+}}$for all $\tau \subseteq \sigma$.

4.1.2 The enriched Coxeter complex $[\mathbb{A} / \overline{\mathbb{G}}]$. - Put $I:=\{1, \cdots, n\}$ and $\mathbb{A}:=\mathbb{Z}^{I} / \mathbb{Z}_{\text {diag. }}$. Two elements $x, y$ in $\mathbb{A}$ are called adjacent if we can find representatives $\tilde{x}, \tilde{y}$ in $\mathbb{Z}^{I}$ such that $\tilde{x}(i) \leqslant \tilde{y}(i) \leqslant \tilde{x}(i)+1$ for all $i \in I$. A subset $\sigma$ of $\mathbb{A}$ is called a simplex if all its pairs of elements are adjacent. We thus get a simplicial complex $\mathbb{A}_{\bullet}$, namely the Coxeter complex of type $\widetilde{A}_{n-1}$. The natural action of the extended affine Weyl group $W:=\mathbb{Z}^{I} \rtimes \mathfrak{S}_{n}$ on $\mathbb{A}$ is simplicial. We will also denote by $W^{0} \subset W$ the (non-extended) affine Weyl group, given by $W^{0}=\left(\mathbb{Z}^{I}\right)^{0} \rtimes \mathfrak{S}_{n} \subset W$ where $\left(\mathbb{Z}^{I}\right)^{0}=\left\{\left(x_{i}\right)_{i \in I}, \sum_{i} x_{i}=0\right\}$. For any simplex $\sigma \in \mathbb{A}$ we put $W_{\sigma}^{0}:=W_{\sigma}^{\dagger} \cap W^{0}$. Then the fixator of $\sigma$ in $W$ decomposes as $W_{\sigma}=W_{\sigma}^{0} \times \mathbb{Z}_{\text {diag }}$ and the quotient $W_{\sigma}^{\dagger} / W_{\sigma}$ embeds in $W /\left(W^{0} \mathbb{Z}_{\text {diag }}\right)$ hence is cyclic of order dividing $n$.

Let $\sigma$ be a simplex in $\mathbb{A}$. If we fix a vertex $x_{0} \in \sigma$ and a representative $\tilde{x}_{0}$ of $x_{0}$, there is a unique ordering $\sigma=\left\{x_{0}, x_{1}, \cdots x_{d}\right\}$ such that we can find representatives $\tilde{x}_{i}: I \longrightarrow \mathbb{Z}$ of $x_{i}$ verifying $\tilde{x}_{0} \leqslant \cdots \leqslant \tilde{x}_{d} \leqslant \tilde{x}_{0}+1$. We get a partition $I=I_{\sigma, 0} \sqcup I_{\sigma, 1} \sqcup \cdots \sqcup I_{\sigma, d}$ of $I$ defined by $I_{\sigma, k}:=\left\{i \in I, \tilde{x}_{k+1}-\tilde{x}_{k}=1\right\}$. Up to cycling the indices $k \mapsto k+1(\bmod d)$, this partition does not depend on the choice of $x_{0}$ and its representative. In particular, the subset $P_{\sigma}=\left\{I_{\sigma, 0}, \cdots, I_{\sigma, d}\right\}$ of the power set $\mathcal{P}(I)$ of $I$ is canonically attached to $\sigma$. We then define a finite group of Lie type

$$
\overline{\mathbb{G}}_{\sigma}:=\prod_{J \in P_{\sigma}} \operatorname{Aut}_{\mathbb{F}}\left(\mathbb{F}^{J}\right) \subset \operatorname{Aut}_{\mathbb{F}}\left(\mathbb{F}^{I}\right)
$$

If $\tau \subseteq \sigma$ is another simplex, then the partition $P_{\sigma}$ refines $P_{\tau}$, so that $\overline{\mathbb{G}}_{\sigma}$ canonically embeds as a (semi-standard) Levi subgroup of $\overline{\mathbb{G}}_{\tau}$. Moreover, if we choose $x_{0} \in \tau$, the associated ordering on $P_{\sigma}$ provides a flag $\mathbb{F}^{I} \supset \mathbb{F}^{I \backslash I_{\sigma, 0}} \supset \cdots \supset \mathbb{F}^{I_{\sigma, d}} \supset 0$ in $\mathbb{F}^{I}$ whose intersection with each $\mathbb{F}^{I_{\tau, k}}$ does not depend on the choice of $x_{0} \in \tau$. We thus get a "canonical" parabolic subgroup $\overline{\mathbb{P}}_{\sigma, \tau}$ of $\overline{\mathbb{G}}_{\tau}$ with Levi component $\overline{\mathbb{G}}_{\sigma}$, and whose unipotent radical we denote by $\overline{\mathbb{U}}_{\sigma, \tau}$.

Now, if $w \in W$, its image $\bar{w} \in \mathfrak{S}_{n}$ under the quotient map $W \rightarrow \mathfrak{S}_{n}$ takes $P_{\sigma}$ to $P_{w \sigma}$, hence the permutation matrix $[\bar{w}] \in \operatorname{Aut}_{\mathbb{F}}\left(\mathbb{F}^{I}\right)$ conjugates $\overline{\mathbb{G}}_{\sigma}$ to $\overline{\mathbb{G}}_{w \sigma}$. We denote by $\bar{w}_{\sigma}: \overline{\mathbb{G}}_{\sigma} \stackrel{\sim}{\longrightarrow} \overline{\mathbb{G}}_{w \sigma}$ the isomorphism thus obtained. When $w \in W_{\sigma}$, the permutation $\bar{w}$ preserves each subset $J \in P_{\sigma}$ of $I$, hence $[\bar{w}] \in \overline{\mathbb{G}}_{\sigma}$. Moreover, the map $w \mapsto[\bar{w}]$ is a group embedding $W_{\sigma}^{0} \hookrightarrow \overline{\mathbb{G}}_{\sigma}$. Finally, if $\tau \subseteq w \sigma$ (i.e. $\left.w \in W_{\sigma \tau}^{\dagger}\right)$, we put

$$
\bar{w}_{\sigma \tau}: \overline{\mathbb{G}}_{\sigma} \stackrel{\bar{w}_{\sigma}}{\longrightarrow} \overline{\mathbb{G}}_{w \sigma} \hookrightarrow \overline{\mathbb{G}}_{\tau}
$$

the composition of the maps just defined. The image $\bar{w}_{\sigma \tau}\left(\overline{\mathbb{G}}_{\sigma}\right)$ is a Levi subgroup of $\overline{\mathbb{G}}_{\tau}$ which is contained in the parabolic subgroup $\overline{\mathbb{P}}_{w \sigma, \tau}=\bar{w}_{\sigma \tau}\left(\overline{\mathbb{G}}_{\sigma}\right) \overline{\mathbb{U}}_{w \sigma, \tau}$. If $v \in W_{\tau \nu}^{\dagger}$ for a third simplex $\nu$, it is easily checked that $\bar{v}_{\tau \nu} \circ \bar{w}_{\sigma \tau}=v \bar{w}_{\sigma \nu}$, and that $\overline{\mathbb{U}}_{v w \sigma, \nu}=\overline{\mathbb{U}}_{v \tau, \nu} . \bar{v}_{\tau \nu}\left(\mathbb{U}_{w \sigma, \tau}\right)$. 
For $\sigma, \tau \in \mathbb{A}$ • we now define

$$
\overline{\mathbb{G}}_{\sigma \tau}^{\dagger}:=\left(\overline{\mathbb{G}}_{\tau} \times W_{\sigma \tau}^{\dagger}\right) / \sim
$$

where

$$
(\bar{g}, w) \sim\left(\bar{g}^{\prime}, w^{\prime}\right) \text { iff } \exists(\bar{u}, v) \in \overline{\mathbb{U}}_{w \sigma, \tau} \times W_{\tau}^{0}, \bar{g}^{\prime}=\bar{g} \bar{u} v \text { and } w^{\prime}=v^{-1} w .
$$

One checks that the composition rule $\left(\overline{\mathbb{G}}_{\nu} \times W_{\tau \nu}^{\dagger}\right) \times\left(\overline{\mathbb{G}}_{\tau} \times W_{\sigma \tau}^{\dagger}\right) \longrightarrow \overline{\mathbb{G}}_{\nu} \times W_{\sigma \nu}^{\dagger}$ given by $\left(\bar{g}_{\nu}, v\right) \circ\left(\bar{g}_{\tau}, w\right)=\left(\bar{g}_{\nu} \bar{v}_{\tau \nu}\left(\bar{g}_{\tau}\right), v w\right)$ descends to a map

$$
\overline{\mathbb{G}}_{\tau \nu}^{\dagger} \times \overline{\mathbb{G}}_{\sigma \tau}^{\dagger} \longrightarrow \overline{\mathbb{G}}_{\sigma \nu}^{\dagger}
$$

that fulfills the necessary associativity property to justify the following definition.

Definition. - We denote by $[\mathbb{A} / \overline{\mathbb{G}}]$ the small category whose set of objects is $\mathbb{A}_{\text {. and the }}$ hom-sets are given by $\operatorname{Hom}(\sigma, \tau)=\overline{\mathbb{G}}_{\sigma \tau}^{\dagger}$.

4.1.3 A functor from $[\mathbb{A} / \overline{\mathbb{G}}]$ to $[B T / \bar{G}]$. - Let us choose a basis of the $F$-vector space $V$. This allows us to identify $V$ to $F^{I}$ and $G$ to $\operatorname{Aut}_{F}\left(F^{I}\right)$. We define a map $\mathbb{A} \stackrel{\iota}{\longrightarrow} B T$ by sending $\tilde{x} \in \mathbb{Z}^{I}$ to the lattice $\mathcal{L}(\tilde{x}):=\bigoplus \varpi^{\tilde{x}(i)} \mathcal{O}_{F} .[i]$ where $\{[i], i \in I\}$ is the canonical basis of $F^{I}$. This

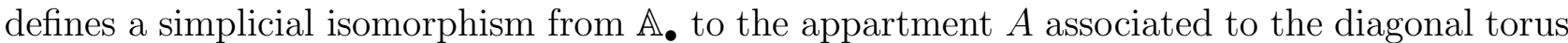
of $G$. Moreover for any simplex $\sigma$ in $\mathbb{A}$ there is a canonical identification $\overline{\mathbb{G}}_{\sigma}=\bar{G}_{\iota \sigma}^{0}:=G_{\iota \sigma}^{0} / G_{\iota \sigma}^{+}$, and if $\tau \subseteq \sigma$ the identification $\overline{\mathbb{G}}_{\tau}=\bar{G}_{\iota \tau}^{0}$ carries $\overline{\mathbb{P}}_{\sigma, \tau}$ to $G_{\iota \sigma}^{0} / G_{\iota \tau}^{+}$and $\overline{\mathbb{U}}_{\sigma, \tau}$ to $G_{\iota \sigma}^{+} / G_{\iota \tau}^{+}$.

We also embed $W$ in $G$ by mapping $w=\left(t, w_{0}\right)$ to the matrix $[t]\left[w_{0}\right]$ where $\left[w_{0}\right]$ is the permutation matrix of $G$ attached to $w_{0}$ and $[t]=\operatorname{diag}\left(\varpi^{t_{1}} \cdots \varpi^{t_{n}}\right)$ if $t=\left(t_{1}, \cdots, t_{n}\right)$. The map $\iota$ is then $W$-equivariant and the map $W_{\iota \sigma}^{0} \longrightarrow \bar{G}_{\iota \sigma}^{0}$ identifies with the map $W_{\sigma}^{0} \longrightarrow \overline{\mathbb{G}}_{\sigma}^{0}$ of the previous paragraph.

4.1.4 Theorem. - The multiplication map $\overline{\mathbb{G}}_{\tau} \times W_{\sigma \tau}^{\dagger}=\bar{G}_{\iota \tau}^{0} \times W_{\iota \sigma, \iota \tau}^{\dagger} \longrightarrow \bar{G}_{\iota \sigma, \iota \tau}^{\dagger}$ induces a bijection $\overline{\mathbb{G}}_{\sigma \tau}^{\dagger} \stackrel{\sim}{\longrightarrow} \bar{G}_{\tau \sigma, \iota \tau}^{\dagger}$. Moreover, the functor

$$
[\mathbb{A} / \overline{\mathbb{G}}] \longrightarrow[B T / \bar{G}]
$$

thus obtained is an equivalence of categories.

Proof. In order to simplify the notation, we write $\sigma$ for $\iota \sigma$ and $\tau$ for $\iota \tau$. In view of the definition of $\overline{\mathbb{G}}_{\sigma \tau}^{\dagger}$ and the foregoing discussion, what need to be proved is that the multiplication map of the theorem induces a bijection

$$
\left(G_{\tau}^{0} \times W_{\sigma \tau}^{\dagger}\right) / \sim \stackrel{\sim}{\longrightarrow} \bar{G}_{\sigma \tau}^{\dagger}
$$

where the equivalence relation is given by

$$
(g, w) \sim\left(g^{\prime}, w^{\prime}\right) \text { iff } \exists(h, u, v) \in G_{\tau}^{+} \times G_{w \sigma}^{+} \times W_{\tau}^{0}, g^{\prime}=h g u v \text { and } w^{\prime}=v^{-1} w .
$$

Since $W_{\tau}^{0}=G_{\tau}^{0} \cap W$, we may simplify this in

$$
(g, w) \sim\left(g^{\prime}, w^{\prime}\right) \text { iff } \exists(h, u) \in G_{\tau}^{+} \times G_{w \sigma}^{+}, g^{\prime} w^{\prime}=h g u w .
$$

But since $G_{\tau}^{+} \subset G_{w \sigma}^{+}$and $G_{w \sigma}^{+}=w G_{\sigma}^{+} w^{-1}$, we also have

$$
(g, w) \sim\left(g^{\prime}, w^{\prime}\right) \text { iff } \exists u \in G_{\sigma}^{+}, g^{\prime} w^{\prime}=g w u .
$$


Therefore the multiplication map descends to

$$
\left(G_{\tau}^{0} \times W_{\sigma \tau}^{\dagger}\right) / \sim \stackrel{\sim}{\longrightarrow}\left(G_{\tau}^{0} W_{\sigma \tau}^{\dagger}\right) / G_{\sigma}^{+} \subset \bar{G}_{\sigma \tau}^{\dagger}
$$

and we are left to prove that the inclusion $G_{\tau}^{0} W_{\sigma \tau}^{\dagger} \subset G_{\sigma \tau}^{\dagger}$ is an equality. So let $g$ be an element in $G_{\sigma \tau}^{\dagger}$, that is $g \sigma \supset \tau$. Since $\tau \in A_{\bullet}$ we can find $g_{\tau} \in G_{\tau}^{0}$ such that $g_{\tau} g \sigma \in A_{\bullet}$. Then, since $\sigma \in A_{\bullet}$ too, there is $w \in W$ such that $w \sigma=g_{\tau} g \sigma$, so that we have $g^{-1} g_{\tau}^{-1} w \in G_{\sigma}^{\dagger}$. Using the equality $G_{\sigma}^{\dagger}=G_{\sigma}^{0} W_{\sigma}^{\dagger}$, we finally write $g$ in the form $g_{\tau}^{-1} w g_{\sigma}^{-1} w_{\sigma}^{-1}$ which belongs to $G_{\tau}^{0} W_{\sigma \tau}^{\dagger}$ since $w G_{\sigma}^{0} w^{-1} \subset G_{\tau}^{0}$.

We thus have obtained a fully faithful functor $[\mathbb{A} / \overline{\mathbb{G}}] \longrightarrow[B T / \bar{G}]$. But since any simplex in $B T$ is conjugate to some simplex in $A$ under $G$, and therefore isomorphic to this simplex in $[B T / \bar{G}]$, this functor is also essentially surjective.

4.1.5 Corollary. - Let $\mathcal{C}$ be any category. The restriction functor $\mathcal{E} \mapsto \mathcal{E} \circ \iota$ is an equivalence of categories between the category of $\mathcal{C}$-valued level $0 G$-equivariant coefficient systems on $B T$ and the category of functors $[\mathbb{A} / \overline{\mathbb{G}}] \longrightarrow \mathcal{C}$.

As in Definition 4.1.1, let us say that a functor $\mathcal{E}:[\mathbb{A} / \overline{\mathbb{G}}] \longrightarrow \mathcal{C}$ is cartesian if $\mathcal{E}\left(\overline{1}_{\sigma \tau}\right)$ is an isomorphism $\mathcal{E}_{\sigma} \stackrel{\sim}{\longrightarrow} \mathcal{E}_{\tau}^{\mathbb{U}_{\sigma \tau}}$ for all $\sigma \supset \tau$. The above equivalence clearly preserves cartesian objects on both sides. Using now 3.1.5 and 3.1.6, we get :

4.1.6 Corollary. - Let $R$ be any commutative ring with $p \in R^{\times}$. The functor $V \mapsto$ $\left(V^{G_{\iota \sigma}^{+}}\right)_{\sigma \in \mathbb{A}}$ is an equivalence of categories between $\operatorname{Rep}_{R}^{0}(G)$ and the category of cartesian functors $[\mathbb{A} / \overline{\mathbb{G}}] \longrightarrow R-\operatorname{Mod}$ (with natural transforms as morphisms).

Note that this implies in particular that the level 0 subcategory $\operatorname{Rep}_{R}^{0}\left(\operatorname{GL}_{n}(F)\right)$ only depends on the residue field $\mathbb{F}$ of $F$ (and $n$ ), up to equivalence. Indeed, the definition of $[\mathbb{A} / \overline{\mathbb{G}}]$ only involves the Coxeter complex associated to the affine Weyl group $W$, and the residual reductive groups $\overline{\mathbb{G}}_{\sigma}, \sigma \in \mathbb{A}$. In order to refine the above corollary for summands $\operatorname{Rep} \frac{s}{\overline{\mathbb{Z}}_{\ell}}(G)$, we enhance the setting a bit.

\subsection{Hecke "algebras" and "modules"}

We refer to the appendix for the language of "rings" (also called ringoids) and "modules" that we will use from now on. As above, $R$ denotes a commutative ring with $p \in R^{\times}$.

4.2.1 The Hecke "algebra" of level 0. - Let us apply the construction of the $R$-algebra of a small category $\mathcal{A}$ as in $\mathrm{A}$.1.1 to the case $\mathcal{A}=[\mathbb{A} / \overline{\mathbb{G}}]$. We get an "R-algebra" $R[\mathbb{A} / \overline{\mathbb{G}}]$ with set of objects $\mathbb{A}_{\text {• }}$ and hom-sets defined by $R[\mathbb{A} / \overline{\mathbb{G}}](\sigma, \tau)=R\left[\overline{\mathbb{G}}_{\sigma \tau}^{\dagger}\right]$. We wish to use Corollary 4.1 .6 in order to see $\operatorname{Rep}_{R}^{0}(G)$ as a category of left modules over $R[\mathbb{A} / \overline{\mathbb{G}}]$. Indeed, this corollary provides us with a full and faithful functor

$$
V \mapsto \mathcal{V}, \operatorname{Rep}_{R}^{0}(G) \longrightarrow R[\mathbb{A} / \overline{\mathbb{G}}]-\operatorname{Mod}
$$

with $\mathcal{V}_{\sigma}:=V^{G_{\iota \sigma}^{+}}$and the action $\mathcal{V}_{\sigma} \otimes_{R} R\left[\overline{\mathbb{G}}_{\sigma \tau}^{\dagger}\right] \longrightarrow \mathcal{V}_{\tau}$ obtained by $R$-linear extension of the action of $\overline{\mathbb{G}}_{\sigma \tau}^{\dagger}$ induced by that of $G$ on $V$ via the isomorphisms $\overline{\mathbb{G}}_{\sigma \tau}^{\dagger} \stackrel{\sim}{\longrightarrow} \bar{G}_{\sigma \tau}^{\dagger}$. The image of this functor is formed by " $R[\mathbb{A} / \overline{\mathbb{G}}]$-modules" which are cartesian as functors $[\mathbb{A} / \overline{\mathbb{G}}] \longrightarrow R-\operatorname{Mod}$ (as 
defined above Corollary 4.1.6). So we need to understand what this property means in terms of " $R[\mathbb{A} / \overline{\mathbb{G}}]$-modules".

Define $W_{\sigma \tau}^{0}:=W_{\sigma \tau}^{\dagger} \cap W^{0}$ and $\overline{\mathbb{G}}_{\sigma \tau}^{0}:=\left(\overline{\mathbb{G}}_{\tau} \times W_{\sigma \tau}^{0}\right) / \sim$ where the equivalence relation is the same as in the definition of $\overline{\mathbb{G}}_{\sigma \tau}^{\dagger}$ (so, in particular we have $\overline{\mathbb{G}}_{\sigma}^{0}=\overline{\mathbb{G}}_{\sigma}$ ). We get a category $[\mathbb{A} / \overline{\mathbb{G}}]^{0}$ and an " $R$-algebra" $R[\mathbb{A} / \overline{\mathbb{G}}]^{0}$ which is naturally a "sub- $R$-algebra" of $R[\mathbb{A} / \overline{\mathbb{G}}]$. Indeed any "module" over $R[\mathbb{A} / \overline{\mathbb{G}}]$ restricts canonically to a "module" over $R[\mathbb{A} / \overline{\mathbb{G}}]^{0}$. We refer to Definition A.1.5 for the notion of Cartesian "module".

Theorem. - The functor $V \mapsto \mathcal{V}$ above is an equivalence of categories from $\operatorname{Rep}_{R}^{0}(G)$ to the category of left $R[\mathbb{A} / \overline{\mathbb{G}}]$-modules that are cartesian as $R[\mathbb{A} / \overline{\mathbb{G}}]^{0}$-modules.

Proof. Let $\sigma, \tau$ be two facets such that $W_{\sigma \tau}^{0}$ is non empty, and fix $w \in W_{\sigma \tau}^{0}$. The next lemma tells us that $W_{\sigma \tau}^{0}=W_{\tau}^{0} w$, and this implies in turn an identification of $\overline{\mathbb{G}}_{\tau} \times \overline{\mathbb{G}}_{\sigma}^{\text {opp }}$-sets

$$
\overline{\mathbb{G}}_{\sigma \tau}^{0}=\overline{\mathbb{G}}_{\tau} / \overline{\mathbb{U}}_{w \sigma, \tau}
$$

where $g_{\sigma} \in \overline{\mathbb{G}}_{\sigma}$ acts on the right by multiplication by $\bar{w}_{\sigma}\left(g_{\sigma}\right) \in \overline{\mathbb{G}}_{w \sigma}$. For any left " $R[\mathbb{A} / \mathbb{G}]^{0}$ module" $\mathcal{M}$, this implies an isomorphism

$$
\operatorname{Hom}_{R\left[\overline{\mathbb{G}}_{\tau}^{0}\right]}\left(R\left[\overline{\mathbb{G}}_{\sigma \tau}^{0}\right], \mathcal{M}_{\tau}\right) \stackrel{\sim}{\longrightarrow} \mathcal{M}_{\tau}^{\overline{\mathbb{U}}_{w \sigma, \tau}}
$$

which identifies the map $a_{\sigma \tau}^{*}$ of A.1.5 with the map

$$
\mathcal{M}_{\sigma} \stackrel{\sim}{\longrightarrow} \mathcal{M}_{w \sigma} \longrightarrow \mathcal{M}_{\tau}^{\overline{\mathbb{U}}_{w \sigma, \tau}}
$$

where the first map is $\mathcal{M}\left(\bar{w}_{\sigma}\right)$ and the second one is $\mathcal{M}\left(\overline{1}_{w \sigma, \tau}\right)$ (the image by the functor $\mathcal{M}$ of the inclusion $\left.1_{w \sigma, \tau}: w \sigma \subseteq \tau\right)$. In particular, we see that $\mathcal{M}$ is cartesian as a functor $[\mathbb{A} / \overline{\mathbb{G}}]^{0} \longrightarrow R-$ Mod if and only if it is cartesian as a " $R[\mathbb{A} / \overline{\mathbb{G}}]^{0}$-module".

Lemma. - Given two chambers $\delta \supset \sigma$ and $\gamma \supset \tau$, the multiplication in $W$ induces two bijections $W_{\tau}^{0} \times\left(W_{\delta \gamma}^{\dagger} \cap W_{\sigma \tau}^{\dagger}\right) \stackrel{\sim}{\longrightarrow} W_{\sigma \tau}^{\dagger}$ and $W_{\tau}^{0} \times\left(W_{\delta \gamma}^{0} \cap W_{\sigma \tau}^{0}\right) \stackrel{\sim}{\longrightarrow} W_{\sigma \tau}^{0}$. Moreover, $W_{\delta \gamma}^{0}$ is a singleton.

Proof. Since $W_{\tau}^{0}$ acts simply transitively on the set of chambers that contain $\tau$, the multiplication map gives a bijection $W_{\tau}^{0} \times W_{\delta \gamma}^{\dagger} \stackrel{\sim}{\longrightarrow} W_{\delta \tau}^{\dagger}$. But we have $W_{\sigma \tau}^{\dagger} \subset W_{\delta \tau}^{\dagger}$, and this bijection thus restricts to a bijection $W_{\tau}^{0} \times\left(W_{\delta \gamma}^{\dagger} \cap W_{\sigma \tau}^{\dagger}\right) \stackrel{\sim}{\longrightarrow} W_{\sigma \tau}^{\dagger}$ and to a similar bijection with $\circ$ instead of $\dagger$. Finally, $W^{0}$ acts simply transitively on the set of all chambers, so $W_{\delta \gamma}^{0}$ is a singleton.

4.2.2 Hecke "algebras" attached to semisimple conjugacy classes. - Now, let $s \in \mathrm{GL}_{n}(\mathbb{F})$ be a semisimple conjugacy class of order invertible in $R$, and assume $R$ is either $\overline{\mathbb{Z}}_{\ell}$ or $\overline{\mathbb{Q}}_{\ell}$. For each $\sigma \in \mathbb{A}$. we get a central idempotent $e_{\sigma}^{s} \in R\left[\overline{\mathbb{G}}_{\sigma}\right]$. Namely, with the notation of $[2.1 .3$ we put

$$
e_{\sigma}^{s}:=\sum_{t} e_{t}^{\overline{\mathbb{G}}_{\sigma}}
$$

where $t$ runs on conjugacy classes in $s \cap \prod_{J \in P_{\sigma}} \operatorname{Aut}_{\mathbb{F}}\left(\mathbb{F}^{J}\right)$ inside $\operatorname{Aut}_{\mathbb{F}}\left(\mathbb{F}^{I}\right)=\mathrm{GL}_{n}(\mathbb{F})$.

Lemma. - The idempotent $e_{\sigma}^{s}$ is central in $R\left[\overline{\mathbb{G}}_{\sigma}^{\dagger}\right]$. Moreover, for any other $\tau \in \mathbb{A}$. we have $e_{\tau}^{s} R\left[\overline{\mathbb{G}}_{\sigma \tau}^{\dagger}\right]=e_{\tau}^{s} R\left[\overline{\mathbb{G}}_{\sigma \tau}^{\dagger}\right] e_{\sigma}^{s}=R\left[\overline{\mathbb{G}}_{\sigma \tau}^{\dagger}\right] e_{\sigma}^{s}$. 
Proof. Any $w \in W_{\sigma}^{\dagger}$ acts on $\overline{\mathbb{G}}_{\sigma}=\prod_{J \in P_{\sigma}} \operatorname{Aut}_{\mathbb{F}}\left(\mathbb{F}^{J}\right)$ by permuting $P_{\sigma}$, and this action is by conjugation by some element in $\operatorname{Aut}_{\mathbb{F}}\left(\mathbb{F}^{I}\right)$. Therefore $w$ normalizes $e_{\sigma}^{s}$. Since $\overline{\mathbb{G}}_{\sigma}^{\dagger}=\overline{\mathbb{G}}_{\sigma} W_{\sigma}^{\dagger}$, the first assertion follows.

For the second one, let $\Omega_{\sigma \tau}^{\dagger}$ be a set of representatives of $W_{\tau} \backslash W_{\sigma \tau}^{\dagger}$. Then we have a decomposition of $\overline{\mathbb{G}}_{\tau} \times \overline{\mathbb{G}}_{\sigma}^{\text {opp }}$ sets

$$
\overline{\mathbb{G}}_{\sigma \tau}^{\dagger}=\bigsqcup_{w \in \Omega_{\sigma \tau}^{\dagger}} \overline{\mathbb{G}}_{\tau} / \mathbb{U}_{w \sigma, \tau},
$$

where $g_{\sigma} \in \overline{\mathbb{G}}_{\sigma}$ acts on the summand $\overline{\mathbb{G}}_{\tau} / \mathbb{U}_{w \sigma, \tau}$ by right multiplication by $\bar{w}_{\sigma \tau}\left(g_{\sigma}\right)$. It follows that

$$
R\left[\overline{\mathbb{G}}_{\sigma \tau}^{\dagger}\right] e_{\sigma}^{s}=\bigoplus_{w \in \Omega_{\sigma \tau}^{\dagger}} R\left[\overline{\mathbb{G}}_{\tau} / \mathbb{U}_{w \sigma, \tau}\right] e_{w \sigma}^{s}=\bigoplus_{w \in \Omega_{\sigma \tau}^{\dagger}} e_{\tau}^{s} R\left[\overline{\mathbb{G}}_{\tau} / \mathbb{U}_{w \sigma, \tau}\right]=e_{\tau}^{s} R\left[\overline{\mathbb{G}}_{\sigma \tau}^{\dagger}\right]
$$

where the second equality is (2.1.5).

The lemma shows that the composition map $R\left[\overline{\mathbb{G}}_{\tau \nu}^{\dagger}\right] \otimes_{R} R\left[\overline{\mathbb{G}}_{\sigma \tau}^{\dagger}\right] \longrightarrow R\left[\overline{\mathbb{G}}_{\sigma \nu}^{\dagger}\right]$ takes the submodule $e_{\nu}^{s} R\left[\overline{\mathbb{G}}_{\tau \nu}^{\dagger}\right] e_{\tau}^{s} \otimes_{R} e_{\tau}^{s} R\left[\overline{\mathbb{G}}_{\sigma \tau}^{\dagger}\right] e_{\sigma}^{s}$ into $e_{\nu}^{s} R\left[\overline{\mathbb{G}}_{\sigma \nu}^{\dagger}\right] e_{\sigma}^{s}$. This justifies the following definition :

Definition. - We denote by $e^{s} R[\mathbb{A} / \overline{\mathbb{G}}]$ the "R-algebra" whose set of objects is $\mathbb{A}_{\text {. }}$ and Hom sets are given by $\operatorname{Hom}(\sigma, \tau)=e_{\tau}^{s} R\left[\overline{\mathbb{G}}_{\sigma \tau}^{\dagger}\right] e_{\sigma}^{s}$. Similarly we have a "R-algebra" $e^{s} R[\mathbb{A} / \overline{\mathbb{G}}]^{0}$.

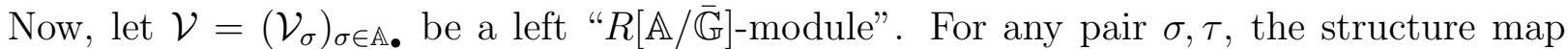
$\mathcal{V}_{\sigma} \otimes_{R} R\left[\overline{\mathbb{G}}_{\sigma \tau}^{\dagger}\right] \longrightarrow \mathcal{V}_{\tau}$ induces a map $e_{\sigma}^{s} \mathcal{V}_{\sigma} \otimes_{R} e_{\tau}^{s} R\left[\overline{\mathbb{G}}_{\sigma \tau}^{\dagger}\right] e_{\sigma}^{s} \longrightarrow e_{\tau}^{s} \mathcal{V}_{\tau}$, which equips the collection $\left(e_{\sigma}^{s} \mathcal{V}_{\sigma}\right)_{\sigma \in \mathbb{A}}$. with a structure of left " $e^{s} R[\mathbb{A} / \overline{\mathbb{G}}]$-module" that we denote by $e^{s} \mathcal{V}$. We have a decomposition $\mathcal{V}=\bigoplus_{s} e^{s} \mathcal{V}$ of $\mathcal{V}$ as a direct sum of functors $\mathbb{A} / \overline{\mathbb{G}} \longrightarrow R$ - Mod. Here $s$ runs over semisimple conjugacy classes in $\mathrm{GL}_{n}(\mathbb{F})$ of order invertible in $R$. In other words we have a categorical decomposition

$$
R[\mathbb{A} / \overline{\mathbb{G}}]-\operatorname{Mod}=\prod_{s} e^{s} R[\mathbb{A} / \overline{\mathbb{G}}]-\operatorname{Mod}
$$

Putting Fact 3.1.5, Lemma 3.2.1 and Theorem 4.2.1 together, we obtain the following result.

Theorem. - The functor $V \mapsto \mathcal{V}$ of 4.2.1 induces an equivalence of categories from $\operatorname{Rep}_{R}^{s}(G)$ to the category of left "es $R[\mathbb{A} / \overline{\mathbb{G}}]$-modules" which are cartesian as "es $R[\mathbb{A} / \overline{\mathbb{G}}]^{0}$-modules".

Since the construction of $e^{s} R[\mathbb{A} / \overline{\mathbb{G}}]$ only involves $\mathbb{F}, n$ and $s$, we infer :

Corollary. - Let $F^{\prime}$ be another local field with residue field isomorphic to $\mathbb{F}$. The categories $\operatorname{Rep}_{R}^{s}\left(\mathrm{GL}_{n}(F)\right)$ and $\operatorname{Rep}_{R}^{s}\left(\mathrm{GL}_{n}\left(F^{\prime}\right)\right)$ are equivalent.

In particular, taking $s=1$ and $F^{\prime}$ an unramified extension of $F$, we have constructed an equivalence of categories such as predicted in 1.1.4 i).

\subsection{Morita equivalence of Hecke "algebras"}

In this section, we assume that $n=n^{\prime} f$ and we denote by $\mathbb{F}^{\prime}$ the extension of $\mathbb{F}$ of degree $f$. To $\left(n^{\prime}, \mathbb{F}^{\prime}\right)$ we associate the enriched Coxeter complex $\left[\mathbb{A}^{\prime} / \overline{\mathbb{G}}^{\prime}\right]$ as in 4.1.2. In particular, $\mathbb{A}^{\prime}=\mathbb{Z}^{I^{\prime}} / \mathbb{Z}_{\text {diag }}$, where $I^{\prime}=\left\{1, \cdots, n^{\prime}\right\}$, and $W^{\prime}=\mathbb{Z}^{I^{\prime}} \rtimes \mathfrak{S}_{n^{\prime}}$. Next, to any semisimple conjugacy 
class $s^{\prime} \in \mathrm{GL}_{n^{\prime}}\left(\mathbb{F}^{\prime}\right)$ with order invertible in $R$, we associate the Hecke " $R$-algebra" $e^{s^{\prime}} R\left[\mathbb{A}^{\prime} / \overline{\mathbb{G}}^{\prime}\right]$ as in 4.2 .2 .

By choosing a basis of $\mathbb{F}^{\prime}$ over $\mathbb{F}$, we get an embedding of $\mathrm{GL}_{n^{\prime}}\left(\mathbb{F}^{\prime}\right)$ into $\mathrm{GL}_{n}(\mathbb{F})$, and we denote by $s$ the unique conjugacy class in $\mathrm{GL}_{n}(\mathbb{F})$ that contains $s^{\prime}$. Note that $s$ does not depend on this choice of basis.

(*) We assume that the centralizer in $\mathrm{GL}_{n}(\mathbb{F})$ of an element of $s^{\prime}$ is contained in $\mathrm{GL}_{n^{\prime}}\left(\mathbb{F}^{\prime}\right)$.

For simplicity, put $\mathcal{R}:=e^{\mathfrak{s}} R[\mathbb{A} / \overline{\mathbb{G}}]$ and $\mathcal{R}^{\prime}:=e^{\mathfrak{s}^{\prime}} R\left[\mathbb{A}^{\prime} / \overline{\mathbb{G}}^{\prime}\right]$. Our aim is to prove that the categories $\mathcal{R}-\operatorname{Mod}$ and $\mathcal{R}^{\prime}-\operatorname{Mod}$ are equivalent, under the above assumption. For this, we will construct a $\left(\mathcal{R}, \mathcal{R}^{\prime}\right)$-bimodule $\mathcal{P}$ and show it induces a Morita equivalence in the sense of Definition A.2.5.

4.3.1 Embedding $\left[\mathbb{A}^{\prime} / W^{\prime}\right]$ in $[\mathbb{A} / W]$. - We fix a surjective map $\pi: I \longrightarrow I^{\prime}$ with fibers of cardinality $f$. Dually, this map induces an embedding $\iota: \mathbb{Z}^{I^{\prime}} \hookrightarrow \mathbb{Z}^{I}$, as well as an embedding $\mathfrak{S}_{n^{\prime}} \hookrightarrow \mathfrak{S}_{n}$ which sends a permutation $w^{\prime}$ to the unique permutation $w=\iota\left(w^{\prime}\right)$ which induces an increasing bijection $\pi^{-1}\left(r^{\prime}\right) \stackrel{\sim}{\longrightarrow} \pi^{-1}\left(w^{\prime}\left(r^{\prime}\right)\right)$ for each $r^{\prime} \in I^{\prime}$. In turn, these two maps induce

- a group embedding $\iota: W^{\prime} \hookrightarrow W$, and

- a simplicial, dimension preserving, embedding $\iota: \mathbb{A}_{\bullet}^{\prime} \hookrightarrow \mathbb{A}_{\bullet}$, which is equivariant with respect to the previous embedding.

Therefore we have obtained an embedding of categories $\left[\mathbb{A}^{\prime} / W^{\prime}\right] \longrightarrow[\mathbb{A} / W]$. Note that for any $\sigma^{\prime} \in \mathbb{A}_{\bullet}^{\prime}$, we have $P_{\iota \sigma^{\prime}}=\pi^{-1}\left(P_{\sigma^{\prime}}\right)$.

4.3.2 Gradings. - Consider the map $\rho: W \longrightarrow \mathbb{Q},\left(\left(x_{1}, \cdots, x_{n}\right), w\right) \mapsto \sum_{i=1}^{n} x_{i}$. Then $\rho$ is a group morphism with kernel $W^{0}$ and image $\frac{1}{n} \mathbb{Z}$. For $i \in \mathbb{Q}$, we will put $W^{i}:=\rho^{-1}(i)$. This notation will propagate to all notation involving $W$, e.g. we put $W_{\sigma \tau}^{i}=W_{\sigma \tau}^{\dagger} \cap W^{i}$. Note that Lemma 4.2.1 tells us that if $W_{\sigma \tau}^{i}$ is not empty, it is a principal homogeneous $W_{\tau}^{0}$-set. In particular, the multiplication map induces a bijection

$$
W_{\tau \nu}^{j} \times_{W_{\tau}^{0}} W_{\sigma \tau}^{i} \stackrel{\sim}{\longrightarrow} W_{\sigma \nu}^{i+j} .
$$

For $\sigma, \tau \in \mathbb{A}_{\bullet}$, the composition $\overline{\mathbb{G}}_{\tau} \times W_{\sigma \tau}^{\dagger} \longrightarrow W_{\sigma \tau}^{\dagger} \stackrel{\rho}{\longrightarrow} \mathbb{Q}$ factors through $\overline{\mathbb{G}}_{\sigma \tau}^{\dagger}$, and we will denote by $\overline{\mathbb{G}}_{\sigma \tau}^{i}$ the inverse image of $i$. Note that $\overline{\mathbb{G}}_{\sigma \tau}^{i}$ is also the quotient set of $\overline{\mathbb{G}}_{\tau} \times W_{\sigma \tau}^{i}$ by the equivalence relation of 4.1.2. If $w \in W_{\sigma \tau}^{i}$, then $\overline{\mathbb{G}}_{\sigma \tau}^{i} \simeq \overline{\mathbb{G}}_{\tau} / \overline{\mathbb{U}}_{w \sigma, \tau}$. In particular we see that the multiplication map induces a bijection

$$
\overline{\mathbb{G}}_{\tau \nu}^{j} \times \overline{\mathbb{G}}_{\tau} \overline{\mathbb{G}}_{\sigma \tau}^{i} \stackrel{\sim}{\longrightarrow} \overline{\mathbb{G}}_{\sigma \nu}^{i+j} .
$$

The disjoint union $\overline{\mathbb{G}}_{\sigma \tau}^{\dagger}=\bigsqcup_{i} \overline{\mathbb{G}}_{\sigma \tau}^{i}$ induces a grading $R\left[\overline{\mathbb{G}}_{\sigma \tau}^{\dagger}\right]=\bigoplus_{i} R\left[\overline{\mathbb{G}}_{\sigma \tau}^{i}\right]$, and in turn a grading $\mathcal{R}_{\sigma, \tau}=\bigoplus_{i} \mathcal{R}_{\sigma, \tau}^{i}$. Note that $\mathcal{R}^{0}=e^{s} R[\mathbb{A} / \overline{\mathbb{G}}]^{0}$ so this notation is consistent with the former one. The multiplication map $\mathcal{R}_{\sigma, \tau} \otimes \mathcal{R}_{\tau, \nu} \longrightarrow \mathcal{R}_{\sigma, \nu}$ takes $\mathcal{R}_{\sigma, \tau}^{i} \otimes \mathcal{R}_{\tau, \nu}^{j}$ into $\mathcal{R}_{\sigma, \nu}^{i+j}$, so we may think of $\mathcal{R}$ as a graded "ring". Actually, the above bijection shows that the same multiplication map induces an isomorphism

$$
\mathcal{R}_{\sigma, \tau}^{i} \otimes_{\mathcal{R}_{\tau}^{0}} \mathcal{R}_{\tau, \nu}^{j} \stackrel{\sim}{\longrightarrow} \mathcal{R}_{\sigma, \nu}^{i+j} .
$$

Similarly, there is a map $\rho^{\prime}: W^{\prime} \longrightarrow \mathbb{Q}$ and we will use similar notation as above for $W^{\prime}$ and $\mathcal{R}^{\prime}$. We have $\rho^{\prime}=\rho \circ \iota$, so that in particular we have $\iota\left(W^{\prime i}\right) \subset W^{i}$. 


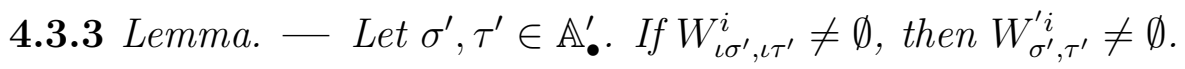

Proof. After maybe conjugating $\sigma^{\prime}$ by some element in $W^{\prime 0}$ we may assume that $\sigma^{\prime}$ and $\tau^{\prime}$ are contained in a chamber $\delta^{\prime}$. Let $c^{\prime}$ be the unique element in $W_{\delta^{\prime}}^{1 / n^{\prime}}$. Then $W_{\sigma^{\prime}, \tau^{\prime}}^{\prime} \neq \emptyset \Leftrightarrow \tau^{\prime} \subset$ $c^{\prime i n^{\prime}} \sigma^{\prime}$, because $W_{\tau^{\prime}}^{0}$ acts transitively on the set of chambers that contain $\tau^{\prime}$. Let us choose a vertex $x_{0}^{\prime} \in \delta^{\prime}$, whence a unique labelling $\delta^{\prime}=\left\{x_{0}^{\prime}, \cdots, x_{n^{\prime}-1}^{\prime}\right\}$, as in 4.1.2. On this labelling, $c^{\prime}$ is the circular permutation. Let us identify $\delta^{\prime}$ to $\mathbb{Z} / n^{\prime} \mathbb{Z}$ via $x_{k}^{\prime} \mapsto k\left(\bmod n^{\prime}\right)$. This identifies $c^{\prime}$ to the map $r \mapsto r+1$. Then $\sigma^{\prime}$ and $\tau^{\prime}$ correspond to subsets $S^{\prime}, T^{\prime}$ of $\mathbb{Z} / n^{\prime} \mathbb{Z}$ and we have

$$
W_{\sigma^{\prime}, \tau^{\prime}}^{\prime i} \neq \emptyset \Leftrightarrow T^{\prime} \subset S^{\prime}+i n^{\prime}
$$

Now let $\delta$ be a chamber in $\mathbb{A}_{\bullet}$ that contains $\iota \delta^{\prime}$. The vertex $x_{0}:=\iota x_{0}^{\prime}$ gives an ordering $\delta=\left\{x_{0}, \cdots, x_{n-1}\right\}$ and we have $\iota x_{k}^{\prime}=x_{k f}$. Let us identify $\delta$ to $\mathbb{Z} / n \mathbb{Z}$ as above. This identifies the inclusion $\iota \delta^{\prime} \subset \delta$ to the multipication by $f$ map $\mathbb{Z} / n^{\prime} \mathbb{Z} \hookrightarrow \mathbb{Z} / n \mathbb{Z}$, hence the simplex $\iota \sigma^{\prime}$ corresponds to $f S^{\prime}$ and the simplex $\iota \tau^{\prime}$ to $f T^{\prime}$. As above, we have

$$
W_{\iota \sigma^{\prime}, \iota \tau^{\prime}}^{i} \neq \emptyset \Leftrightarrow f T^{\prime} \subset f S^{\prime}+i n,
$$

which is equivalent to $T^{\prime} \subset S^{\prime}+i n^{\prime}$ since $n=f n^{\prime}$.

4.3.4 Embedding $\left[\mathbb{A}^{\prime} / \overline{\mathbb{G}}^{\prime}\right]$ in $[\mathbb{A} / \overline{\mathbb{G}}]$. - Next, we fix an $\mathbb{F}$-basis $\left(\alpha_{1}, \cdots, \alpha_{f}\right)$ of $\mathbb{F}^{\prime}$. We associate to it the $\mathbb{F}$-linear isomorphism $\iota: \mathbb{F}^{\prime I^{\prime}} \stackrel{\sim}{\longrightarrow} \mathbb{F}^{I}$ defined as follows. Let $\eta: I^{\prime} \times$ $\{1, \cdots, f\} \stackrel{\sim}{\longrightarrow} I$ be the unique bijection which commutes with the projections to $I^{\prime}$ (i.e. $\pi \circ$ $\eta$ coincides with the first projection $\pi_{1}$ ) and which is increasing on each fiber of $\pi_{1}$. Then we let $\iota$ be the $\mathbb{F}$-linear map which takes $\alpha_{j} e_{i^{\prime}}^{\prime}$ to $e_{\eta\left(i^{\prime}, j\right)}$, where $\left(e_{i}\right)_{i \in I}$ and $\left(e_{i^{\prime}}^{\prime}\right)_{i^{\prime} \in I^{\prime}}$ are the respective canonical basis of $\mathbb{F}^{I}$ and $\mathbb{F}^{I^{\prime}}$. This isomorphism induces in turn an embedding $\iota_{*}: \operatorname{Aut}_{\mathbb{F}^{\prime}}\left(\mathbb{F}^{\prime I^{\prime}}\right) \hookrightarrow \operatorname{Aut}_{\mathbb{F}}\left(\mathbb{F}^{I}\right)$ and we note that this embedding restricts to the embedding $\iota: \mathfrak{S}_{n^{\prime}} \hookrightarrow \mathfrak{S}_{n}$ defined earlier.

For all $J^{\prime} \subset I^{\prime}$, the isomorphism $\iota$ restricts to $\mathbb{F}^{\prime J^{\prime}} \stackrel{\sim}{\longrightarrow} \mathbb{F}^{\pi^{-1}\left(J^{\prime}\right)}$, and therefore, for all $\sigma^{\prime} \in \mathbb{A}_{\bullet}^{\prime}$, $\iota_{*}$ takes $\overline{\mathbb{G}}_{\sigma^{\prime}}^{\prime}$ into $\overline{\mathbb{G}}_{\iota \sigma^{\prime}}$, compatibly with the previously defined $W^{\prime} \hookrightarrow W$. In other words we now have an embedding of categories $\left[\mathbb{A}^{\prime} / \overline{\mathbb{G}}^{\prime}\right] \hookrightarrow[\mathbb{A} / \overline{\mathbb{G}}]$.

4.3.5 Varieties. - As explained in 2.2.1, we can now see $\overline{\mathbb{G}}_{\sigma^{\prime}}$ as a "twisted" Levi subgroups of $\overline{\mathbb{G}}_{\iota \sigma^{\prime}}$ with a "canonical" choice of a parabolic subgroup, and therefore we get a Deligne-Lusztig variety $Y_{\sigma^{\prime}}$ with an action of $\overline{\mathbb{G}}_{\sigma} \times \overline{\mathbb{G}}_{\sigma^{\prime}}^{\text {opp }}$. We then define

$$
\mathcal{P}_{\sigma^{\prime}}^{0}:=H_{c}^{\operatorname{dim}}\left(Y_{\sigma^{\prime}}, \Lambda\right) e_{s^{\prime}}^{\overline{\mathbb{G}}_{\sigma}^{\prime}} \text { in } \mathcal{R}_{\iota \sigma^{\prime}}^{0} \otimes_{\Lambda}\left(\mathcal{R}_{\sigma^{\prime}}^{\prime 0}\right)^{\mathrm{opp}}-\operatorname{Mod}
$$

4.3.6 Lemma. — There is a family of isomorphisms $\left(\phi_{\sigma^{\prime}, \tau^{\prime}}\right)_{\sigma^{\prime}, \tau^{\prime} \in \mathbb{A}_{\bullet}^{\prime}}$ of $\left(\mathcal{R}_{\iota \tau^{\prime}}^{0}, \mathcal{R}_{\sigma^{\prime}}^{\prime 0}\right)$-bimodules

$$
\phi_{\sigma^{\prime}, \tau^{\prime}}: \mathcal{P}_{\sigma^{\prime}}^{0} \otimes_{\mathcal{R}_{\iota \sigma^{\prime}}^{0}} \mathcal{R}_{\iota \sigma^{\prime}, \iota \tau^{\prime}} \stackrel{\sim}{\longrightarrow} \mathcal{R}_{\sigma^{\prime} \tau^{\prime}}^{\prime} \otimes_{\mathcal{R}_{\tau^{\prime}}^{\prime 0}} \mathcal{P}_{\tau^{\prime}}^{0}
$$


which satisfy the following transitivity property. For any other $\nu^{\prime} \in \mathbb{A}_{\bullet}^{\prime}$, the following diagram

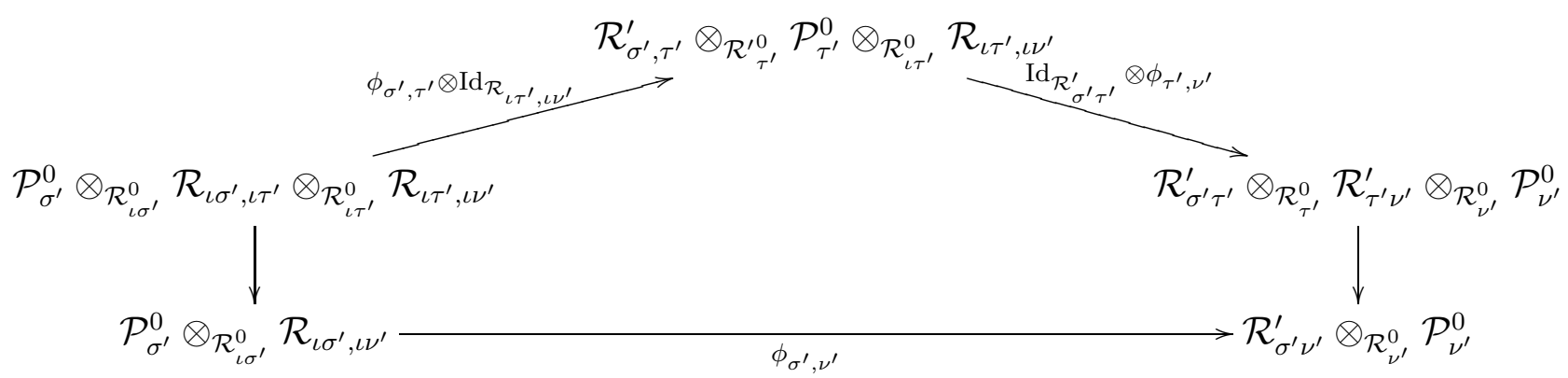

is commutative, where the vertical maps are given by composition in $\mathcal{R}$ and $\mathcal{R}^{\prime}$.

Proof. Step 1. Assume that $\tau^{\prime} \subset \sigma^{\prime}$. In this case we are in the setting of 2.2.3. Namely, the parabolic subgroup $\overline{\mathbb{P}}_{\iota \sigma^{\prime}, \iota \tau^{\prime}}=\overline{\mathbb{G}}_{\iota \sigma^{\prime}} \overline{\mathbb{U}}_{\iota \sigma, \iota \tau^{\prime}}$ of $\overline{\mathbb{G}}_{\iota \tau^{\prime}}$ is defined by the image under $\iota_{*}$ of the flag that defines the parabolic subgroup $\overline{\mathbb{P}}_{\sigma^{\prime}, \tau^{\prime}}^{\prime}=\overline{\mathbb{G}}_{\sigma^{\prime}}^{\prime} \overline{\mathbb{U}}_{\sigma^{\prime}, \tau^{\prime}}^{\prime}$ of $\overline{\mathbb{G}}_{\tau^{\prime}}^{\prime}$. In particular we have $\overline{\mathbb{P}}_{\sigma^{\prime}, \tau^{\prime}}^{\prime}=$ $\overline{\mathbb{P}}_{\iota \sigma^{\prime}, \iota \tau^{\prime}} \cap \overline{\mathbb{G}}_{\tau^{\prime}}^{\prime}$. Since moreover we have $\mathcal{R}_{\iota \sigma^{\prime}, \iota \tau^{\prime}}^{0}=R\left[\overline{\mathbb{G}}_{\iota \tau^{\prime}} / \overline{\mathbb{U}}_{\iota \sigma^{\prime}, \iota \tau^{\prime}}\right]$ and $\mathcal{R}_{\sigma^{\prime}, \tau^{\prime}}^{\prime 0}=R\left[\overline{\mathbb{G}}_{\tau^{\prime}}^{\prime} / \overline{\mathbb{U}}_{\sigma^{\prime}, \tau^{\prime}}^{\prime}\right]$, Proposition 2.2.5 provides us with an isomorphism of $\left(\mathcal{R}_{\iota \tau^{\prime}}^{0}, \mathcal{R}_{\sigma^{\prime}}^{\prime 0}\right)$-bimodules 3

$$
\phi_{\sigma^{\prime}, \tau^{\prime}}^{0}: \mathcal{P}_{\sigma^{\prime}}^{0} \otimes_{\mathcal{R}_{\iota \sigma^{\prime}}^{0}} \mathcal{R}_{\iota \sigma^{\prime}, \iota \tau^{\prime}}^{0} \stackrel{\sim}{\longrightarrow} \mathcal{R}_{\sigma^{\prime} \tau^{\prime}}^{\prime 0} \otimes_{\mathcal{R}_{\tau^{\prime}}^{\prime 0}} \mathcal{P}_{\tau^{\prime}}^{0}
$$

Moreover, if $\nu^{\prime} \subset \tau^{\prime}$, the following diagram commutes by statement ii) of the same proposition

$$
\begin{aligned}
& \mathcal{P}_{\sigma^{\prime}}^{0} \otimes_{\mathcal{R}_{\iota \sigma^{\prime}}^{0}} \mathcal{R}_{\iota \sigma^{\prime}, \iota \tau^{\prime}}^{0} \otimes_{\mathcal{R}_{\iota \tau^{\prime}}^{0}} \mathcal{R}_{\iota \tau^{\prime}, \iota \nu^{\prime}}^{0} \stackrel{\left(\operatorname{Id}_{\mathcal{R}_{\sigma^{\prime} \tau^{\prime}}^{0}} \otimes \phi_{\tau^{\prime}, \nu^{\prime}}^{0}\right) \circ\left(\phi_{\sigma^{\prime}, \tau^{\prime}}^{0} \otimes \mathrm{Id}_{\mathcal{R}_{\iota \tau^{\prime}, \iota \nu^{\prime}}^{0}}\right)}{\longrightarrow} \mathcal{R}_{\sigma^{\prime} \tau^{\prime}}^{0} \otimes_{\mathcal{R}_{\tau^{\prime}}^{0}} \mathcal{R}_{\tau^{\prime} \nu^{\prime}}^{0} \otimes_{\mathcal{R}_{\nu^{\prime}}^{0}} \mathcal{P}_{\nu^{\prime}}^{0} \\
& \simeq \downarrow \\
& \mathcal{P}_{\sigma^{\prime}}^{0} \otimes_{\mathcal{R}_{\iota \sigma^{\prime}}^{0}} \mathcal{R}_{\iota \sigma^{\prime}, \iota \nu^{\prime}}^{0} \longrightarrow \mathcal{R}_{\sigma^{\prime} \nu^{\prime}}^{0} \otimes_{\mathcal{R}_{\nu^{\prime}}^{0}} \mathcal{P}_{\nu^{\prime}}^{0}
\end{aligned}
$$

Note that in this diagram the vertical maps are isomorphisms, so that we can express it as an equality

$$
\phi_{\sigma^{\prime}, \nu^{\prime}}^{0}=\left(\operatorname{Id}_{\mathcal{R}_{\sigma^{\prime} \tau^{\prime}}^{0}} \otimes \phi_{\tau^{\prime}, \nu^{\prime}}^{0}\right) \circ\left(\phi_{\sigma^{\prime}, \tau^{\prime}}^{0} \otimes \operatorname{Id}_{\mathcal{R}_{\iota \tau^{\prime}, \iota \nu^{\prime}}^{0}}\right) .
$$

Step 2. Assume that $\tau^{\prime} \in W^{\prime i} \cdot \sigma^{\prime}$ for some $i \in \mathbb{Q}$. Pick $w \in W^{\prime i}$, such that $\tau^{\prime}=w \sigma^{\prime}$. We also denote by $w$ the image $\iota(w)$ of $w$ in $W$. Conjugation by the permutation matrix $[\bar{w}]$ in $\operatorname{Aut}_{\mathbb{F}}\left(\mathbb{F}^{I}\right)$ induces an isomorphism of varieties $\bar{w}: Y_{\sigma^{\prime}} \stackrel{\sim}{\longrightarrow} Y_{\tau^{\prime}}$ which is equivariant with respect to the isomorphisms $\bar{w}_{\sigma^{\prime}}: \overline{\mathbb{G}}_{\sigma^{\prime}} \stackrel{\sim}{\longrightarrow} \overline{\mathbb{G}}_{\tau^{\prime}}$ and $\bar{w}_{\iota \sigma^{\prime}}: \overline{\mathbb{G}}_{l \sigma^{\prime}} \stackrel{\sim}{\longrightarrow} \overline{\mathbb{G}}_{L \tau^{\prime}}$. Recall that the latter isomorphisms are also given by conjugation under $[w]$ in $\operatorname{Aut}_{\mathbb{F}}\left(\mathbb{F}^{I}\right)$, and coincide with the "outer-conjugacy" by $w$ seen as an isomorphism $\sigma^{\prime} \stackrel{\sim}{\longrightarrow} \tau^{\prime}$ in the category $\left[\mathbb{A}^{\prime} / \overline{\mathbb{G}}^{\prime}\right]$, resp. $\iota \sigma^{\prime} \stackrel{\sim}{\longrightarrow} \iota \tau^{\prime}$ in the category $[\mathbb{A} / \overline{\mathbb{G}}]$. Recall also that the maps

$$
\begin{aligned}
\overline{\mathbb{G}}_{\tau^{\prime}, \sigma^{\prime}}^{\prime} & \rightarrow \overline{\mathbb{G}}_{\tau^{\prime}}^{\prime} \\
x^{\prime} & \mapsto w x^{\prime}
\end{aligned} \text { and } \begin{aligned}
\overline{\mathbb{G}}_{\iota \sigma^{\prime}, L \tau^{\prime}}^{i} & \rightarrow \overline{\mathbb{G}}_{\iota \tau^{\prime}} \\
x & \mapsto x w^{-1}
\end{aligned}
$$

\footnotetext{
${ }^{3}$ Note that the tensor products have been switched, because of our convention to write "left modules" on the right (in accordance with the composition of morphisms in a category rather than with the action of a usual ring on a module, see A.1.2
} 
are bijections. It follows that the map $\left(x^{\prime}, y, x\right) \mapsto\left(x w^{-1}\right) \cdot(\bar{w} y) \cdot\left(w x^{\prime}\right)$ is a $\overline{\mathbb{G}}_{\iota \tau^{\prime}}^{\prime} \times \overline{\mathbb{G}}_{\tau^{\prime}}^{\prime \text { opp }}$ equivariant isomorphism

$$
\alpha_{w}: \overline{\mathbb{G}}_{\tau^{\prime}, \sigma^{\prime}}^{\prime-i} \times_{\overline{\mathbb{G}}_{\sigma^{\prime}}^{\prime}} Y_{\sigma^{\prime}} \times_{\overline{\mathbb{G}}_{\iota \sigma^{\prime}}} \overline{\mathbb{G}}_{\iota \sigma^{\prime}, \iota \tau^{\prime}}^{i} \stackrel{\sim}{\longrightarrow} Y_{\tau^{\prime}} .
$$

Moreover, let $v \in W_{\sigma^{\prime}}^{0}$. We have $\alpha_{w v}\left(x^{\prime}, y, x\right)=\alpha_{w}\left(v x^{\prime}, \bar{v} y, x v^{-1}\right)$. But the automorphism $\bar{v}$ of $Y_{\sigma^{\prime}}$ is given by the action of $\left([v],[v]^{-1}\right) \in \overline{\mathbb{G}}_{\iota \sigma^{\prime}} \times \overline{\mathbb{G}}_{\sigma^{\prime}}$. Therefore, $\left(v x^{\prime}, \bar{v} y, x v^{-1}\right)=\left(x^{\prime}, y, x\right)$ in the contracted product above, and hence we have $\alpha_{w v}=\alpha_{w}$. Since $W_{\sigma^{\prime}}^{\prime}$ acts simply transitively on $W_{\sigma^{\prime} \tau^{\prime}}^{\prime i}$, we see that $\alpha_{w}$ does not depend on the choice of $w$. We denote it by $\alpha_{i}$. Taking the contracted product on the left with $\overline{\mathbb{G}}_{\sigma^{\prime}, \tau^{\prime}}^{\prime i}$ and passing to cohomology we get an isomorphism of $\left(\mathcal{R}_{\iota \tau^{\prime}}^{0}, \mathcal{R}_{\sigma^{\prime}}^{\prime 0}\right)$-bimodules

$$
\phi_{\sigma^{\prime}, \tau^{\prime}}^{i}: \mathcal{P}_{\sigma^{\prime}}^{0} \otimes_{\mathcal{R}_{\iota \sigma^{\prime}}^{0}} \mathcal{R}_{\iota \sigma^{\prime}, \iota \tau^{\prime}}^{i} \stackrel{\sim}{\longrightarrow} \mathcal{R}_{\sigma^{\prime} \tau^{\prime}}^{i} \otimes_{\mathcal{R}_{\tau^{\prime}}^{\prime 0}} \mathcal{P}_{\tau^{\prime}}^{0}
$$

These isomorphisms compose as expected. Namely, taking into account that the multiplication map induces isomorphisms like $\mathcal{R}_{\sigma, \tau}^{i} \otimes_{\mathcal{R}_{\tau}^{0}} \mathcal{R}_{\tau, \nu}^{j} \stackrel{\sim}{\longrightarrow} \mathcal{R}_{\sigma, \nu}^{i+j}$, we have for any $\nu^{\prime} \in W^{\prime j} \tau^{\prime}$ the following equality

$$
\phi_{\sigma^{\prime}, \nu^{\prime}}^{i+j}=\left(\operatorname{Id}_{\mathcal{R}_{\sigma^{\prime} \tau^{\prime}}^{i}}^{i} \otimes \phi_{\tau^{\prime}, \nu^{\prime}}^{j}\right) \circ\left(\phi_{\sigma^{\prime}, \tau^{\prime}}^{i} \otimes \operatorname{Id}_{\mathcal{R}_{\iota \tau^{\prime}, \iota \nu^{\prime}}^{j}}\right) .
$$

Step 3.Compatibility between steps 1 and 2. Here we pick two simplices $\tau^{\prime} \subset \sigma^{\prime}$ and an element $w \in W^{\prime i}$. In order to simplify the notation a bit, we put $\sigma=\iota \sigma^{\prime}$ and $\tau=\iota \tau^{\prime}$. We have a commutative diagram

$$
\begin{aligned}
& \mathcal{P}_{\sigma^{\prime}}^{0} \otimes_{\mathcal{R}_{\sigma}^{0}} \mathcal{R}_{\sigma, \tau}^{0} \longrightarrow{\phi_{\sigma^{\prime}, \tau^{\prime}}^{0}}_{\mathcal{R}_{\sigma^{\prime}, \tau^{\prime}}^{\prime}}^{0} \otimes_{\mathcal{R}_{\tau^{\prime}}^{\prime 0}} \mathcal{P}_{\tau^{\prime}}^{0} \\
& {[w] \otimes\left(w \circ-\circ w^{-1}\right) \downarrow \simeq \quad \simeq \downarrow\left(w \circ-\circ w^{-1}\right) \otimes[w]} \\
& \mathcal{P}_{w \sigma^{\prime}}^{0} \otimes_{\mathcal{R}_{w \sigma}^{0}}^{0} \mathcal{R}_{w \sigma, w \tau}^{0} \underset{\phi_{w \sigma^{\prime}, w \tau^{\prime}}^{0}}{\longrightarrow} \mathcal{R}_{w \sigma^{\prime}, w \tau^{\prime}}^{\prime 0} \otimes_{\mathcal{R}^{\prime}{ }_{w \tau^{\prime}}^{0}}^{0} \mathcal{P}_{w \tau^{\prime}}^{0}
\end{aligned}
$$

where the vertical maps are given by conjugation by $[w]$ in $\operatorname{Aut}_{\mathbb{F}}\left(\mathbb{F}^{I}\right)$. As in step 2, these vertical maps are equivariant with respect to conjugation by $[w]$ and we make them equivariant by tensoring the first line on the left by $\mathcal{R}_{w \sigma^{\prime}, \sigma^{\prime}}^{\prime-i}$ and on the right by $\mathcal{R}_{\tau, w \tau}^{i}$, as follows :

$$
\begin{aligned}
& \mathcal{R}_{w \sigma^{\prime}, \sigma^{\prime}}^{\prime-i} \otimes_{\mathcal{R}_{\sigma^{\prime}}^{0}} \mathcal{P}_{\sigma^{\prime}}^{0} \otimes_{\mathcal{R}_{\sigma}^{0}} \mathcal{R}_{\sigma, \tau}^{0} \otimes_{\mathcal{R}_{\tau}^{0}} \mathcal{R}_{\tau, w \tau}^{i} \stackrel{\mathrm{Id} \otimes \phi_{\sigma^{\prime}, \tau^{\prime}}^{0} \otimes \mathrm{Id}}{\longrightarrow} \mathcal{R}_{w \sigma^{\prime}, \sigma^{\prime}}^{\prime-i} \otimes_{\mathcal{R}_{\sigma^{\prime}}^{\prime 0}} \mathcal{R}_{\sigma^{\prime}, \tau^{\prime}}^{\prime 0} \otimes_{\mathcal{R}_{\tau^{\prime}}^{\prime 0}} \mathcal{P}_{\tau^{\prime}}^{0} \otimes_{\mathcal{R}_{\tau}^{0}} \mathcal{R}_{\tau, w \tau}^{i} \\
& w \otimes[w] \otimes\left(w \circ-\circ w^{-1}\right) \otimes w^{-1}|\simeq \quad \simeq| w \otimes\left(w \circ-\circ w^{-1}\right) \otimes[w] \otimes w^{-1} \\
& \mathcal{P}_{w \sigma^{\prime}}^{0} \otimes_{\mathcal{R}_{w \sigma}^{0}}^{0} \mathcal{R}_{w \sigma, w \tau}^{0} \longrightarrow \mathcal{R}_{w \sigma^{\prime}, w \tau^{\prime}}^{\prime 0} \otimes_{\mathcal{R}^{\prime \prime}{ }_{w \tau^{\prime}}^{0}, w \tau^{\prime}}^{0} \mathcal{P}_{w \tau^{\prime}}^{0}
\end{aligned}
$$

Now we tensor on the left the whole diagram by $\mathcal{R}_{\sigma^{\prime}, w \sigma^{\prime}}^{i}$ and we simplify terms using isomorphisms of the type $\mathcal{R}_{\sigma \tau}^{i} \otimes_{\mathcal{R}_{\tau}^{0}} \mathcal{R}_{\tau \nu}^{j} \stackrel{\sim}{\longrightarrow} \mathcal{R}_{\sigma \nu}^{i+j}$ given by the multiplication. We get a commutative diagram

$$
\begin{aligned}
& \mathcal{P}_{\sigma^{\prime}}^{0} \otimes_{\mathcal{R}_{\sigma}^{0}} \mathcal{R}_{\sigma, w \tau}^{i} \longrightarrow{\phi_{\sigma^{\prime}, \tau^{\prime}}^{0} \otimes \mathrm{Id}_{\mathcal{R}_{\tau, w \tau}}^{i}}_{\mathcal{R}_{\sigma^{\prime}, \tau^{\prime}}^{\prime 0} \otimes_{\mathcal{R}_{\tau^{\prime}}^{\prime 0}}} \mathcal{P}_{\tau^{\prime}}^{0} \otimes_{\mathcal{R}_{\tau}^{0}} \mathcal{R}_{\tau, w \tau}^{i} \\
& \phi_{\sigma^{\prime}, w \sigma^{\prime}}^{i} \otimes \operatorname{Id}_{\mathcal{R}_{w \sigma, w \tau}^{0}} \downarrow \mid \quad \operatorname{Id}_{\mathcal{R}_{w \sigma^{\prime}, w \tau^{\prime}}^{0}} \otimes \phi_{\tau^{\prime}, w \tau^{\prime}}^{i} \\
& \mathcal{R}_{\sigma^{\prime}, w \sigma^{\prime}}^{\prime i} \otimes_{\mathcal{R}^{\prime}{ }_{w \sigma^{\prime}}} \mathcal{P}_{w \sigma^{\prime}}^{0} \otimes_{\mathcal{R}_{w \sigma}^{0}} \mathcal{R}_{w \sigma, w \tau}^{0} \stackrel{\operatorname{Id}_{\mathcal{R}_{\sigma^{\prime}, w \sigma^{\prime}}^{\prime i}} \otimes \phi_{w \sigma^{\prime}, w \tau^{\prime}}^{i}}{\longrightarrow} \mathcal{R}_{\sigma^{\prime}, w \tau^{\prime}}^{\prime i} \otimes_{\mathcal{R}^{\prime}{ }_{w \tau^{\prime}}}^{0} \mathcal{P}_{w \tau^{\prime}}^{0}
\end{aligned}
$$


Note that the objects and morphisms in this diagram only depend on $w \sigma^{\prime}$ and $w \tau^{\prime}$, not on $w$.

Step 4. The desired maps. Let now $\sigma^{\prime}, \tau^{\prime} \in \mathbb{A}^{\prime}$. be any pair of simplices. Assume that $W_{\sigma^{\prime}, \tau^{\prime}}^{i} \neq \emptyset$. So there is a simplex $\nu^{\prime} \in W^{\prime i} \sigma^{\prime}$ such that $\tau^{\prime} \subset \nu^{\prime}$. Consider the composition

$$
\left(\operatorname{Id}_{\mathcal{R}_{\sigma^{\prime} \nu^{\prime}}^{\prime i}}^{i} \otimes \phi_{\nu^{\prime}, \tau^{\prime}}^{0}\right) \circ\left(\phi_{\sigma^{\prime}, \nu^{\prime}}^{i} \otimes \operatorname{Id}_{\mathcal{R}_{\iota \nu^{\prime}, \iota \tau^{\prime}}^{0}}\right): \mathcal{P}_{\sigma^{\prime}}^{0} \otimes_{\mathcal{R}_{\iota \sigma^{\prime}}}^{0} \mathcal{R}_{\iota \sigma^{\prime}, \iota \tau^{\prime}}^{i} \stackrel{\sim}{\longrightarrow} \mathcal{R}_{\sigma^{\prime} \tau^{\prime}}^{i} \otimes_{\mathcal{R}_{\tau^{\prime}}^{\prime \prime}} \mathcal{P}_{\tau^{\prime}}^{0}
$$

We will show it is independent of the choice of $\nu^{\prime}$. So, let $\mu^{\prime}$ be another simplex in $W^{\prime} \sigma^{\prime}$ which contains $\tau^{\prime}$. By Step 2 we have a factorization $\phi_{\sigma^{\prime}, \mu^{\prime}}^{i}=\left(\operatorname{Id}_{\mathcal{R}_{\sigma^{\prime}, \nu^{\prime}}^{i}} \otimes \phi_{\nu^{\prime}, \mu^{\prime}}^{0}\right) \circ\left(\phi_{\sigma^{\prime}, \nu^{\prime}}^{i} \otimes \operatorname{Id}_{\mathcal{R}_{\iota \nu^{\prime}, \iota \mu^{\prime}}^{0}}\right)$. Thanks to this factorization, we are left to prove the following equality

$$
\phi_{\nu^{\prime}, \tau^{\prime}}^{0}=\left(\operatorname{Id}_{\mathcal{R}_{\nu^{\prime}, \mu^{\prime}}^{\prime 0}}^{0} \otimes \phi_{\mu^{\prime}, \tau^{\prime}}^{0}\right) \circ\left(\phi_{\nu^{\prime}, \mu^{\prime}}^{0} \otimes \operatorname{Id}_{\mathcal{R}_{\iota \mu^{\prime}, \iota \tau^{\prime}}^{0}}\right)
$$

Now, since $\mu^{\prime} \in W^{\prime 0} \nu^{\prime}$ and both contain $\tau^{\prime}$, there is $w \in W_{\tau^{\prime}}^{\prime 0}$ such that $\mu^{\prime}=w \nu^{\prime}$. Applying the last diagram of Step 3, with $\left(\nu^{\prime}, \tau^{\prime}\right)$ instead of $\left(\sigma^{\prime}, \tau^{\prime}\right)$ and $i=0$, and taking into account that $w \tau^{\prime}=\tau^{\prime}$, we get exactly the desired equality.

Step 5. Transitivity. Pick three simplices $\sigma^{\prime}, \tau^{\prime}, \nu^{\prime}$. Suppose that $W_{\sigma^{\prime} \tau^{\prime}}^{\prime i}$ and $W_{\tau^{\prime} \nu^{\prime}}^{\prime j}$ are non-empty and pick $w$, resp. $v$, in these sets. We then have a diagram

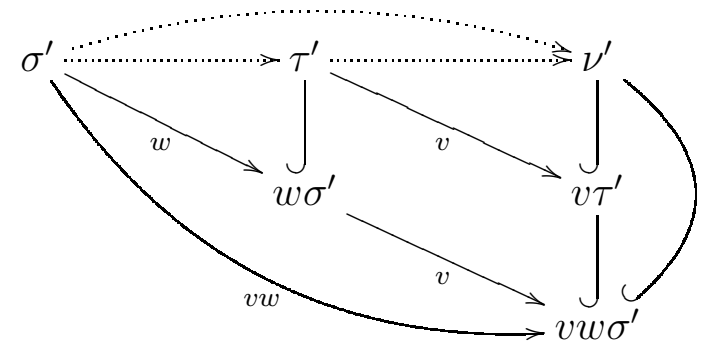

We want to check that the morphisms associated to the dotted arrows in Step 4 compose as expected $\phi_{\sigma^{\prime}, \nu^{\prime}}^{i+j}=\left(\operatorname{Id}_{\mathcal{R}_{\sigma^{\prime} \tau^{\prime}}^{i}} \otimes \phi_{\tau^{\prime}, \nu^{\prime}}^{j}\right) \circ\left(\phi_{\sigma^{\prime}, \tau^{\prime}}^{i} \otimes \operatorname{Id}_{\mathcal{R}_{\iota \tau^{\prime}, \iota \nu^{\prime}}^{j}}\right)$. By definition, each one of them is the composition of the morphism associated to the diagonal arrow in Step 2 and the one associated to the vertical arrow in Step 1, along the corresponding "triangle". We saw in Step 2 that the morphisms associated to diagonal arrows compose as expected. We saw in Step 1 that the morphisms associated to vertical arrows compose as expected. Therefore it is sufficient to check that the diagram of morphisms associated to the parallelogram commutes. But this is exactly what Step 3 tells us.

Step 6. Conclusion. Recall the decompositions $\mathcal{R}_{\iota \sigma^{\prime}, \iota \tau^{\prime}}=\bigoplus_{i \in \mathbb{Q}} \mathcal{R}_{\iota \sigma^{\prime}, \iota \tau^{\prime}}^{i}$ and $\mathcal{R}_{\sigma^{\prime}, \tau^{\prime}}^{\prime}=$ $\bigoplus_{i \in \mathbb{Q}} \mathcal{R}_{\sigma^{\prime}, \tau^{\prime}}^{i}$. Define $\phi_{\sigma^{\prime}, \tau^{\prime}}:=\bigoplus_{i \in \frac{1}{n} \mathbb{Z}} \phi_{\sigma^{\prime}, \tau^{\prime}}^{i}$, where we agree that $\phi_{\sigma^{\prime}, \tau^{\prime}}^{i}=0$ whenever $W_{\sigma^{\prime} \tau^{\prime}}^{\prime i}=\emptyset$ (and in particular if $i \notin \frac{1}{n^{\prime}} \mathbb{Z}$ ). The only point that remains to be checked is that $\phi_{\sigma^{\prime}, \tau^{\prime}}$ is actually an isomorphism, and for this we need to show that for all $i \in \mathbb{Q}$, we have $W_{\iota \sigma^{\prime}, \iota \tau^{\prime}}^{i} \neq$ $\emptyset \Rightarrow W_{\sigma^{\prime}, \tau^{\prime}}^{\prime i} \neq \emptyset$. But this is Lemma 4.3.3.

4.3.7 Construction of a $\left(\mathcal{R}, \mathcal{R}^{\prime}\right)$-bimodule. - For any $\sigma^{\prime} \in \mathbb{A}_{\bullet}^{\prime}$ and $\sigma \in \mathbb{A}$ • we put

$$
\mathcal{P}_{\sigma^{\prime}, \sigma}:=\mathcal{P}_{\sigma^{\prime}}^{0} \otimes_{\mathcal{R}_{\iota \sigma^{\prime}}^{0}} \mathcal{R}_{\iota \sigma^{\prime}, \sigma}
$$


The composition maps $\mathcal{R}_{\iota \sigma^{\prime}, \sigma} \otimes \mathcal{R}_{\sigma, \tau} \longrightarrow \mathcal{R}_{\iota \sigma^{\prime}, \tau}$ induce an obvious left $\mathcal{R}$-module structure on the collection $\left(\mathcal{P}_{\sigma^{\prime}, \sigma}\right)_{\sigma \in \mathbb{A}_{\bullet}}$. On the other hand, define a map $\mathcal{R}_{\sigma^{\prime}, \tau^{\prime}}^{\prime} \otimes \mathcal{P}_{\tau^{\prime}, \tau} \longrightarrow \mathcal{P}_{\sigma^{\prime}, \tau}$ as the following composition of maps :

$$
\begin{aligned}
\mathcal{R}_{\sigma^{\prime}, \tau^{\prime}}^{\prime} \otimes \mathcal{P}_{\tau^{\prime}, \tau} & \longrightarrow \mathcal{R}_{\sigma^{\prime}, \tau^{\prime}}^{\prime} \otimes_{\mathcal{R}_{\tau^{\prime}}^{0}} \mathcal{P}_{\tau^{\prime}, \tau}=\mathcal{R}_{\sigma^{\prime}, \tau^{\prime}}^{\prime} \otimes_{\mathcal{R}_{\tau^{\prime}}^{0}} \mathcal{P}_{\tau^{\prime}}^{0} \otimes_{\mathcal{R}_{\iota \tau^{\prime}}^{0}} \mathcal{R}_{\iota \tau^{\prime}, \tau} \\
& \stackrel{\longrightarrow}{\longrightarrow} \mathcal{P}_{\sigma^{\prime}}^{0} \otimes_{\mathcal{R}_{\iota \sigma^{\prime}}^{0}} \mathcal{R}_{\iota \sigma^{\prime}, \iota \tau^{\prime}} \otimes_{\mathcal{R}_{\iota \tau^{\prime}}^{0}} \mathcal{R}_{\iota \tau^{\prime}, \tau} \\
& \longrightarrow \mathcal{P}_{\sigma^{\prime}}^{0} \otimes_{\mathcal{R}_{\iota \sigma^{\prime}}^{0}} \mathcal{R}_{\iota \sigma^{\prime}, \tau}=\mathcal{P}_{\sigma^{\prime}, \tau}
\end{aligned}
$$

In the second line, the isomorphism is $\phi_{\sigma^{\prime}, \tau^{\prime}}^{-1} \otimes \operatorname{Id}_{\mathcal{R}_{\iota \tau^{\prime}, \tau}}$. The big diagram in Lemma 4.3.6 insures that these action maps are transitive, so that we get a right $\mathcal{R}^{\prime}$-module structure on the collection $\left(\mathcal{P}_{\tau^{\prime}, \tau}\right)_{\tau^{\prime} \in \mathbb{A}_{\bullet}^{\prime}}$. On the other hand this structure clearly commutes with the left action of $\mathcal{R}$, so we have constructed a $\left(\mathcal{R}, \mathcal{R}^{\prime}\right)$-bimodule structure on the collection $\left(\mathcal{P}_{\sigma^{\prime}, \sigma}\right)_{\sigma^{\prime}, \mathbb{A}_{\bullet}^{\prime}, \sigma \in \mathbb{A}_{\bullet}}$, in the sense of A.1.6. We denote it by $\mathcal{P}$ and we refer to Definition A.2.5 for the notion of Morita equivalence in this context.

\subsubsection{Theorem. - The bimodule $\mathcal{P}$ induces a Morita equivalence between $\mathcal{R}$ and $\mathcal{R}^{\prime}$.}

Proof. We show that the hypothesis of Proposition A.2.6 are satisfied. First of all, for all $\sigma^{\prime} \in \mathbb{A}_{\bullet}^{\prime}$, the $\mathcal{R}$-module $\sigma \mapsto \mathcal{P}_{\sigma^{\prime}, \sigma}$ is, by construction, induced from $\iota \sigma^{\prime}$ (in the sense of Definition A.1.4). Next, the isomorphisms $\phi_{\sigma^{\prime}, \tau^{\prime}}: \mathcal{P}_{\sigma^{\prime}, l \tau^{\prime}} \stackrel{\sim}{\longrightarrow} \mathcal{R}_{\sigma^{\prime}, \tau^{\prime}} \otimes_{\mathcal{R}_{\tau^{\prime}}^{0}} \mathcal{P}_{\tau^{\prime}}^{0}$ and $\phi_{\tau^{\prime}, \tau^{\prime}}: \mathcal{P}_{\tau^{\prime}, l \tau^{\prime}} \stackrel{\sim}{\longrightarrow}$ $\mathcal{R}_{\tau^{\prime}} \otimes_{\mathcal{R}_{\tau^{\prime}}^{0}} \mathcal{P}_{\tau^{\prime}}^{0}$ combine to an isomorphism $\mathcal{P}_{\sigma^{\prime}, l \tau^{\prime}} \stackrel{\sim}{\longrightarrow} \mathcal{R}_{\sigma^{\prime}, \tau^{\prime}} \otimes_{\mathcal{R}_{\tau^{\prime}}} \mathcal{P}_{\tau^{\prime}, l \tau^{\prime}}$, which tells us that $\sigma^{\prime} \mapsto \mathcal{P}_{\sigma^{\prime}, \iota \tau^{\prime}}$ is induced from $\tau^{\prime}$. Therefore, hypothesis i) of A.2.6 is satisfied. Now, condition ii) of A.2.6 is that $\mathcal{P}_{\sigma^{\prime}, \iota \sigma^{\prime}}$ should induce a Morita equivalence between $\mathcal{R}_{\iota \sigma^{\prime}}$ and $\mathcal{R}_{\sigma^{\prime}}^{\prime}$. To show this, consider the evaluation map for any $\mathcal{R}_{\iota \sigma^{\prime}}$-module $M$

$$
\mathcal{P}_{\sigma^{\prime}, \iota \sigma^{\prime}} \otimes_{\mathcal{R}_{\sigma^{\prime}}} \operatorname{Hom}_{\mathcal{R}_{\iota \sigma^{\prime}}^{\prime}}\left(\mathcal{P}_{\sigma^{\prime}, \iota \sigma^{\prime}}, M\right) \longrightarrow M
$$

Since $\mathcal{P}_{\sigma^{\prime}, \iota \sigma^{\prime}}=\mathcal{P}_{\sigma^{\prime}}^{0} \otimes_{\mathcal{R}_{\iota \sigma^{\prime}}^{0}} \mathcal{R}_{\iota \sigma^{\prime}} \simeq \mathcal{R}_{\sigma^{\prime}}^{\prime} \otimes_{\mathcal{R}_{\sigma^{\prime}}^{\prime 0}} \mathcal{P}_{\sigma^{\prime}}^{0}$, it coincides with the evaluation map

$$
\mathcal{P}_{\sigma^{\prime}}^{0} \otimes_{\mathcal{R}_{\sigma^{\prime}}^{0}} \operatorname{Hom}_{\mathcal{R}_{\iota \sigma^{\prime}}^{0}}\left(\mathcal{P}_{\sigma^{\prime}}^{0}, M\right) \longrightarrow M
$$

which is indeed an isomorphism since $\mathcal{P}_{\sigma^{\prime}}^{0}$ induces a Morita equivalence between $\mathcal{R}_{\iota \sigma^{\prime}}^{0}$ and $\mathcal{R}_{\sigma^{\prime}}^{0}$. Similarly, the adjunction map

$$
M^{\prime} \longrightarrow \operatorname{Hom}_{\mathcal{R}_{\iota \sigma^{\prime}}}\left(\mathcal{P}_{\sigma^{\prime}, \iota \sigma^{\prime}}, M^{\prime} \otimes_{\mathcal{R}_{\sigma^{\prime}}^{\prime}} \mathcal{P}_{\sigma^{\prime}, \iota \sigma^{\prime}}\right)
$$

is an isomorphism for any $\mathcal{R}_{\sigma^{\prime}}^{\prime}$-module $M^{\prime}$, hence we have the desired Morita equivalence at $\sigma^{\prime}$.

It remains to prove condition iii) of A.2.6. To this end, we need to prove that if the idempotent $e_{\sigma}^{s}$ in $R\left[\overline{\mathbb{G}}_{\sigma}\right]$ is non zero, then $\sigma$ is conjugate to a simplex of the form $\iota \sigma^{\prime}$. Now, $e_{\sigma}^{s}$ is non zero if and only if the conjugacy class of $s$ in $\operatorname{Aut}_{\mathbb{F}}\left(\mathbb{F}^{I}\right)$ intersects $\mathbb{G}_{\sigma}$. Since the centralizer of $s$ is contained in Aut $_{\mathbb{F}^{\prime}}\left(\mathbb{F}^{\prime I^{\prime}}\right)$, this means that $\mathbb{G}_{\sigma}$ contains a maximal torus isomorphic to one of $\operatorname{Aut}_{\mathbb{F}^{\prime}}\left(\mathbb{F}^{\prime I^{\prime}}\right)$ and therefore any $J \in \mathcal{P}_{\sigma}$ has cardinality divisible by $f$. It follows that we can find $\bar{w} \in \mathfrak{S}_{n}$ such that $\bar{w}\left(\mathcal{P}_{\sigma}\right)=\mathcal{P}_{\bar{w} \sigma}$ is the inverse image of a partition of $I^{\prime}$. Now choose a vertex $x_{0} \in \bar{w} \sigma$, whence an ordering $\bar{w} \sigma=\left\{x_{0}, \cdots, x_{d}\right\}$. We can find $t \in \mathbb{Z}^{I}$ such that $t x_{0} \in \iota\left(\mathbb{A}^{\prime}\right)$. Then the fact that $\bar{w}\left(\mathcal{P}_{\sigma}\right)=\mathcal{P}_{\bar{w} \sigma}$ is the inverse image of a partition of $I^{\prime}$ implies inductively on $i$ that each $t x_{i}$ is also in $\iota\left(\mathbb{A}^{\prime}\right)$, so that finally $t \bar{w} \sigma \in \iota\left(\mathbb{A}_{\bullet}^{\prime}\right)$. 
Now we need to prove that the equivalences of categories given by this theorem preserve the property of being cartesian as $\mathcal{R}^{0}$-modules (resp. as $\mathcal{R}^{\prime 0}$-modules). To this end, it is useful to express directly the equivalence in terms of $\mathcal{R}^{0}$-modules and $\mathcal{R}^{\prime 0}$-modules. Let us thus consider

$$
\mathcal{P}_{\sigma^{\prime}, \sigma}^{0}:=\mathcal{P}_{\sigma^{\prime}}^{0} \otimes_{\mathcal{R}_{\iota \sigma^{\prime}}^{0}} \mathcal{R}_{\iota \sigma^{\prime}, \sigma}^{0}
$$

for all $\sigma^{\prime} \in \mathbb{A}^{\prime}$ and $\sigma \in \mathbb{A}_{\text {. }}$. Exactly as in 4.3.7, this collection of $R$-modules has a clear left action by $\mathcal{R}^{0}$, and we get a right $\mathcal{R}^{\prime 0}$-action using the 0 -weight part of the isomorphisms $\phi_{\sigma^{\prime}, \tau^{\prime}}$ of Lemma 4.3.6. Therefore this collection $\mathcal{P}^{0}=\left(\mathcal{P}_{\sigma^{\prime}, \sigma}^{0}\right)_{\sigma^{\prime} \in \mathbb{A}_{\bullet}^{\prime}, \sigma \in \mathbb{A}_{\bullet}}$ has a $\left(\mathcal{R}^{0}, \mathcal{R}^{\prime 0}\right)$-bimodule structure.

4.3.9 Theorem. - $\mathcal{P}^{0}$ induces a Morita equivalence between $\mathcal{R}^{0}$ and $\mathcal{R}^{\prime 0}$. Moreover :

i) the associated equivalences of categories commute with those of Theorem 4.3.8 via the restriction functors from $\mathcal{R}$-modules (resp. $\mathcal{R}^{\prime}$-modules) to $\mathcal{R}^{0}$-modules (resp. $\mathcal{R}^{\prime \prime}$-modules).

ii) the associated equivalences of categories preserve cartesian modules.

Proof. The fact that $\mathcal{P}^{0}$ induces a Morita equivalence follows from Proposition A.2.6, exactly as in Theorem 4.3.8. The only change is that we have to choose the $t$ in $\left(\mathbb{Z}^{I}\right)^{0}$, at the end of that proof. Consequently, also ii) follows from Proposition A.2.6.

To see i), consider the obvious morphism of $\left(\mathcal{R}^{0}, \mathcal{R}^{\prime 0}\right)$-bimodules $\mathcal{P}^{0} \longrightarrow \mathcal{P}_{\mid \mathcal{R}^{0} \times \mathcal{R}^{\prime 0}}$. This induces the following morphism of $\mathcal{R}^{\prime 0}$-modules, natural in the $\mathcal{R}$-module $\mathcal{M}$

$$
\operatorname{Hom}_{\mathcal{R}}(\mathcal{P}, \mathcal{M}) \longrightarrow \operatorname{Hom}_{\mathcal{R}^{0}}\left(\mathcal{P}^{0}, \mathcal{M}\right)
$$

Let $\sigma^{\prime} \in \mathbb{A}_{\text {. }}$. The map $\operatorname{Hom}_{\mathcal{R}}(\mathcal{P}, \mathcal{M})_{\sigma^{\prime}} \longrightarrow \operatorname{Hom}_{\mathcal{R}^{0}}\left(\mathcal{P}^{0}, \mathcal{M}\right)_{\sigma^{\prime}}$ is isomorphic, by Example A.1.8, to the restriction map $\operatorname{Hom}_{\mathcal{R}_{\iota \sigma^{\prime}}}\left(\mathcal{P}_{\sigma^{\prime}, \iota \sigma^{\prime}}, \mathcal{M}_{\iota \sigma^{\prime}}\right) \longrightarrow \operatorname{Hom}_{\mathcal{R}_{\iota \sigma^{\prime}}^{0}}\left(\mathcal{P}_{\sigma^{\prime}, \iota \sigma^{\prime}}^{0}, \mathcal{M}_{\iota \sigma^{\prime}}\right)$ which is bijective since $\mathcal{P}_{\sigma^{\prime}, \iota \sigma^{\prime}} \simeq \mathcal{P}_{\sigma^{\prime}, \iota \sigma^{\prime}}^{0} \otimes_{\mathcal{R}_{\iota \sigma^{\prime}}^{0}} \mathcal{R}_{\iota \sigma^{\prime}}$. Therefore, the displayed morphism is an isomorphism of $\mathcal{R}^{\prime 0}$-modules.

On the other hand, we also have a morphism of $\mathcal{R}^{0}$-modules, natural in the $\mathcal{R}^{\prime}$-module $\mathcal{M}^{\prime}$

$$
\mathcal{P}^{0} \otimes_{\mathcal{R}^{\prime 0}} \mathcal{M}^{\prime} \longrightarrow \mathcal{P} \otimes_{\mathcal{R}^{\prime}} \mathcal{M}^{\prime}
$$

For any $\sigma^{\prime} \in \mathbb{A}_{\bullet}$, the map $\left(\mathcal{P}^{0} \otimes_{\mathcal{R}^{\prime}} \mathcal{M}^{\prime}\right)_{\iota \sigma^{\prime}} \longrightarrow\left(\mathcal{P} \otimes_{\mathcal{R}^{\prime}} \mathcal{M}^{\prime}\right)_{\iota \sigma^{\prime}}$ is isomorphic, by Lemma A.1.11 to the map $\mathcal{M}_{\sigma^{\prime}}^{\prime} \otimes_{\mathcal{R}_{\sigma^{\prime}}^{\prime}} \mathcal{P}_{\sigma^{\prime}, \iota \sigma^{\prime}}^{0} \longrightarrow \mathcal{M}_{\sigma^{\prime}}^{\prime} \otimes_{\mathcal{R}^{\prime}{ }_{\sigma^{\prime}}} \mathcal{P}_{\sigma^{\prime}, \iota \sigma^{\prime}}$ which is bijective since $\mathcal{P}_{\sigma^{\prime}, \iota \sigma^{\prime}} \simeq \mathcal{R}_{\sigma^{\prime}}^{\prime} \otimes_{\mathcal{R}^{\prime \prime} \sigma^{\prime}} \mathcal{P}_{\sigma^{\prime}, \iota \sigma^{\prime}}^{0}$. Since any simplex $\sigma$ with $\mathcal{R}_{\sigma} \neq 0$ is conjugate to some $\iota \sigma^{\prime}$ (see the proof of the last theorem), this shows that the second displayed map is an isomorphism of $\mathcal{R}^{0}$-modules.

\section{A "Rings" and "modules"}

\section{A.1 Generalities}

A.1.1 "Rings". - In this paper, a "ring" is a small category $\mathcal{R}$ such that all Homsets $\mathcal{R}(x, y)$ are abelian groups, and all composition maps $\mathcal{R}(x, y) \times \mathcal{R}(y, z) \longrightarrow \mathcal{R}(x, z)$, $(f, g) \mapsto g \circ f$ are bilinear, thus defining linear maps $\mathcal{R}(x, y) \otimes \mathcal{R}(y, z) \longrightarrow \mathcal{R}(x, z)$ that satisfy the usual transitivity properties. Such gadgets appear in the literature under several 
other names, like "ringoids", "rings with several objects", or "small preadditive categories", or "small categories enriched over the tensor category of abelian groups". Note that for any $x \in \mathcal{R}$, the abelian group $\mathcal{R}(x):=\mathcal{R}(x, x)$ is an ordinary ring with unit. However, this ring is allowed to be the zero ring, in which case $x$ is called a "zero object".

More generally, if $R$ is a commutative ring, an " $R$-algebra" will be a small category $\mathcal{R}$ such that all Hom-sets $\mathcal{R}(x, y)$ are $R$-modules, and all composition maps are $R$-bilinear, thus defining linear maps $\mathcal{R}(x, y) \otimes_{R} \mathcal{R}(y, z) \longrightarrow \mathcal{R}(x, z)$ that satisfy the usual transitivity properties.

If $\mathcal{A}$ is any small category, we denote by $R[\mathcal{A}]$ the following " $R$-algebra". Its objects are those of $\mathcal{A}$ and Hom-sets are the free $R$-modules on Hom-sets of $\mathcal{A}$, i.e. $R[\mathcal{A}](x, y):=R[\mathcal{A}(x, y)]$. If we think of $\mathcal{A}$ as a "monoid with many objects", then $R[\mathcal{A}]$ is the $R$-algebra of that monoid.

A.1.2 "Modules". - Let $\mathcal{R}$ be an " $R$-algebra". A left " $\mathcal{R}$-module" $\mathcal{M}$ is an $R$-linear functor $\mathcal{M}: \mathcal{R} \longrightarrow R$ - Mod. It thus consists in a collection $(\mathcal{M}(x))_{x \in \mathcal{R}}$ of $R$-modules, together with $R$-linear maps $\mathcal{M}(x) \otimes_{R} \mathcal{R}(x, y) \longrightarrow \mathcal{M}(y), m \otimes f \mapsto \mathcal{M}(f)(x)$ that define an action in the sense that the following diagrams should be commutativet :

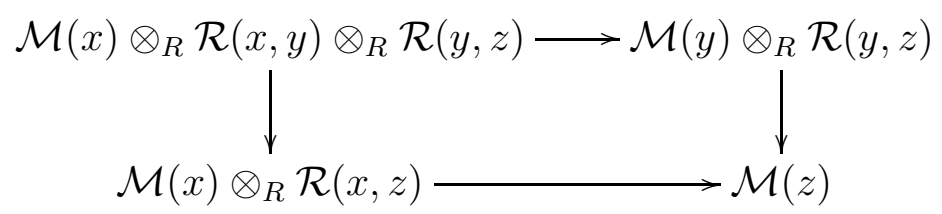

With natural transforms as morphisms, left " $\mathcal{R}$-modules" form an $R$-linear abelian category which we will denote by $\mathcal{R}-\operatorname{Mod}$. Note that $\mathcal{M}(x)=\{0\}$ for any zero object $x$.

Similarly, a right " $\mathcal{R}$-module" will be a contravariant functor $\mathcal{M}: \mathcal{R} \longrightarrow R$ - Mod. These objects again form an abelian $R$-linear category that we will denote by $\operatorname{Mod}-\mathcal{R}$. Note that for any $R$-module $M$, the collection $\left(\operatorname{Hom}_{R}(\mathcal{M}(x), M)\right)_{x \in \mathcal{R}}$ gets a structure of left $\mathcal{R}$-module, that we denote by $\operatorname{Hom}_{R}(\mathcal{M}, M)$.

If $\mathcal{A}$ is a small category, then any functor (resp. contravariant functor) $\mathcal{E}: \mathcal{A} \longrightarrow R-\operatorname{Mod}$ canonically extends to a left (resp. right) " $R[\mathcal{A}]$-module".

A.1.3 Restriction and (co)induction. - For any object $x$, we have an obvious restriction functor $\mathcal{M} \mapsto \mathcal{M}(x), \mathcal{R}-\operatorname{Mod} \longrightarrow \mathcal{R}(x)-\operatorname{Mod}$. In the other direction, we can induce a left $\mathcal{R}(x)$-module $M$ to an $\mathcal{R}$-module $\operatorname{Ind}_{x} M$ by putting

$$
\operatorname{Ind}_{x} M(y):=M \otimes_{\mathcal{R}(x)} \mathcal{R}(x, y)
$$

for any object $y$, with the action of $\mathcal{R}$ given by the multiplication in $\mathcal{R}$. Similarly, we can coinduce $M$ to an $\mathcal{R}$-module Coind $_{x} M$ defined by

$$
\operatorname{Coind}_{x} M(y):=\operatorname{Hom}_{\mathcal{R}(x)}(\mathcal{R}(y, x), M)
$$

Lemma. - The induction functor (resp. the coinduction functor) is left adjoint (resp. right adjoint) to the restriction functor $\operatorname{Res}_{x}$.

\footnotetext{
${ }^{4}$ Our way of displaying these maps suggest that they should define a right module structure, but in terms of composition of maps, this is really a left module structure. For example the diagram says that $\mathcal{M}(x)$ is a left $\mathcal{R}(x, x)$-module
} 
Proof. For an $\mathcal{R}(x)$-module $M$, we have a tautological isomorphism $\operatorname{Id}_{M} \otimes 1: M \stackrel{\sim}{\longrightarrow} \operatorname{Ind}_{x} M(x)=$ $M \otimes_{\mathcal{R}(x)} \mathcal{R}(x)$, and this defines a natural transform Id $\stackrel{\sim}{\longrightarrow} \operatorname{Res}_{x} \circ \operatorname{Ind}_{x}$. On the other hand, for an $\mathcal{R}$-module $\mathcal{M}$, the action maps $\mathcal{M}(x) \otimes_{R} \mathcal{R}(x, y) \longrightarrow \mathcal{M}(y)$ factor through $\mathcal{M}(x) \otimes_{\mathcal{R}(x)} \mathcal{R}(x, y)$, due to diagram A.1.2, and this defines a natural transform $\operatorname{Ind}_{x} \circ \operatorname{Res}_{x} \longrightarrow$ Id. It is immediately checked that these natural transforms are the unit and counit of an adjunction between Res $_{x}$ and $\operatorname{Ind}_{x}$. The case of Coind $x$ is similar.

There are similar functors $\operatorname{Ind}_{x}$ and $\operatorname{Coind}_{x}$ and similar adjunctions for right modules.

A.1.4 Definition. - We say that a left (resp. right) $\mathcal{R}$-module $\mathcal{M}$ is induced from $x$ if it is isomorphic to some $\operatorname{Ind}_{x} M$, or equivalently if the canonical maps

$$
a_{x, y}: \mathcal{M}(x) \otimes_{\mathcal{R}(x)} \mathcal{R}(x, y) \longrightarrow \mathcal{M}(y)\left(\text { resp. } \mathcal{R}(y, x) \otimes_{\mathcal{R}(x)} \mathcal{M}(x) \longrightarrow \mathcal{M}(y)\right)
$$

are isomorphisms for all $y \in \mathcal{R}$.

Similarly, we say that $\mathcal{M}$ is coinduced from $x$ if it is isomorphic to some $\operatorname{Coind}_{x} M$, or equivalently if the canonical maps

$$
a_{y, x}^{*}: \mathcal{M}(y) \longrightarrow \operatorname{Hom}_{\mathcal{R}(x)}(\mathcal{R}(y, x), \mathcal{M}(x))\left(\text { resp. } \mathcal{M}(y) \longrightarrow \operatorname{Hom}_{\mathcal{R}(x)}(\mathcal{R}(x, y), \mathcal{M}(x))\right)
$$

are isomorphisms for all $y \in \mathcal{R}$.

A.1.5 Definition. - We say that a left $\mathcal{R}$-module $\mathcal{M}$ is cartesian or locally coinduced, if all maps $a_{y, x}^{*}$ as above are isomorphisms, whenever $\mathcal{R}(x, y)$ is non-zero.

A.1.6 "Bimodules". - Let $\mathcal{R}, \mathcal{R}^{\prime}$ be two " $R$-algebras". A " $(\mathcal{R}, \mathcal{R}$ ')-bimodule" is an $R$ bilinear functor $\mathcal{R}^{\prime \text { opp }} \times \mathcal{R} \longrightarrow R$-Mod. Concretely, this consists in a collection $\left(\mathcal{B}\left(x^{\prime}, x\right)\right)_{x \in \mathcal{R}, x^{\prime} \in \mathcal{R}^{\prime}}$ of $R$-modules together with action maps

$$
\mathcal{B}\left(x^{\prime}, x\right) \otimes_{R} \mathcal{R}(x, y) \longrightarrow \mathcal{B}\left(x^{\prime}, y\right) \text { and } \mathcal{R}\left(y^{\prime}, x^{\prime}\right) \otimes_{R} \mathcal{B}\left(x^{\prime}, x\right) \longrightarrow \mathcal{B}\left(y^{\prime}, x\right)
$$

that make each collection $\left(\mathcal{B}\left(x^{\prime}, x\right)\right)_{x \in \mathcal{R}}$ into a left $\mathcal{R}$-module and each collection $\left(\mathcal{B}\left(x^{\prime}, x\right)\right)_{x^{\prime} \in \mathcal{R}^{\prime}}$ into a right $\mathcal{R}^{\prime}$-module, and which "commute" in the sense that the following diagrams are commutative

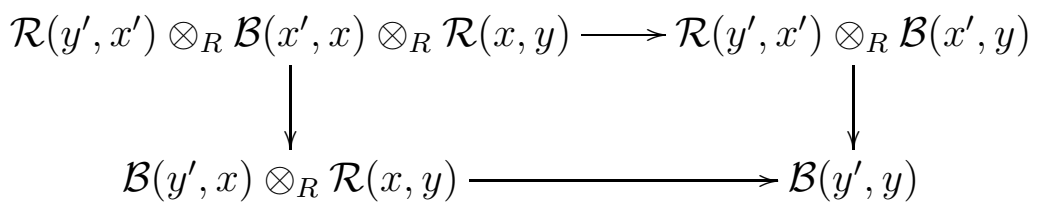

Examples.

i) If $\mathcal{M}$ is a left " $\mathcal{R}$-module" and $\mathcal{M}^{\prime}$ is a right " $\mathcal{R}^{\prime}$-module", we get an " $\left(\mathcal{R}, \mathcal{R}^{\prime}\right)$-bimodule" $\mathcal{M}^{\prime} \otimes_{R} \mathcal{M}$ with underlying collection $\left(\mathcal{M}^{\prime} \otimes_{R} \mathcal{M}\right)\left(x^{\prime}, x\right)=\mathcal{M}^{\prime}\left(x^{\prime}\right) \otimes_{R} \mathcal{M}(x)$

ii) If $\mathcal{B}$ is a " $\left(\mathcal{R}, \mathcal{R}^{\prime}\right)$-bimodule", its "dual" $\mathcal{B}^{*}$ defined by $\mathcal{B}^{*}\left(x, x^{\prime}\right):=\operatorname{Hom}_{R}\left(\mathcal{B}\left(x^{\prime}, x\right), R\right)$ is naturally a " $\left(\mathcal{R}^{\prime}, \mathcal{R}\right)$-bimodule".

iii) If $\mathcal{M}, \mathcal{N}$ are two " $\mathcal{R}$-modules", we get a "( $\mathcal{R}, \mathcal{R})$-bimodule" $\operatorname{Hom}_{R}(\mathcal{M}, \mathcal{N})$ by putting $\operatorname{Hom}_{R}(\mathcal{M}, \mathcal{N})\left(x^{\prime}, x\right):=\operatorname{Hom}_{R}\left(\mathcal{M}\left(x^{\prime}\right), \mathcal{N}(x)\right)$ with the obvious actions. 
A.1.7 Ends and coends. - Let $\mathcal{B}$ be a $(\mathcal{R}, \mathcal{R})$-bimodule. Its end is an $R$-module denoted by $\int_{\mathcal{R}} \mathcal{B}$ and defined by

$$
\int_{\mathcal{R}} \mathcal{B}:=\operatorname{ker}\left(\prod_{x \in \mathcal{R}} \mathcal{B}(x, x) \rightrightarrows \prod_{y, z \in \mathcal{R}} \operatorname{Hom}_{R}(\mathcal{R}(y, z), \mathcal{B}(y, z))\right)
$$

where the first map is a product of the maps $\mathcal{B}(x, x) \longrightarrow \operatorname{Hom}_{R}(\mathcal{R}(x, z), \mathcal{B}(x, z))$ adjoint to $\mathcal{B}(x, x) \otimes_{R} \mathcal{R}(x, z) \longrightarrow \mathcal{B}(x, z)$ and the second map is a product of the maps $\mathcal{B}(x, x) \longrightarrow$ $\operatorname{Hom}_{R}(\mathcal{R}(y, x), \mathcal{B}(y, x))$ adjoint to $\mathcal{R}(y, x) \otimes_{R} \mathcal{B}(x, x) \longrightarrow \mathcal{B}(y, x)$.

Similarly, its coend is an $R$-module denoted by $\int^{\mathcal{R}} \mathcal{B}$ and is defined by

$$
\int^{\mathcal{R}} \mathcal{B}:=\operatorname{coker}\left(\bigoplus_{y, z \in \mathcal{R}} \mathcal{R}(z, y) \otimes_{R} \mathcal{B}(y, z) \rightrightarrows \bigoplus_{x \in \mathcal{R}} \mathcal{B}(x, x)\right)
$$

where the first map is a sum of maps $\mathcal{R}(z, y) \otimes_{R} \mathcal{B}(y, z) \longrightarrow \mathcal{B}(y, y)$ (giving the left modules structure) and the second map is a sum of maps $\mathcal{R}(z, y) \otimes_{R} \mathcal{B}(y, z) \longrightarrow \mathcal{B}(z, z)$ (giving the right module structure).

A.1.8 Example. - If $\mathcal{M}, \mathcal{N}$ are two $\mathcal{R}$-modules, we have $\int_{\mathcal{R}} \mathcal{H} \operatorname{Hom}_{R}(\mathcal{M}, \mathcal{N})=\operatorname{Hom}_{\mathcal{R}}(\mathcal{M}, \mathcal{N})$. We note that when $\mathcal{M}$ is induced from an object $x$, Lemma A.1.3 tells us that the canonical $\operatorname{map} \int_{\mathcal{R}} \mathcal{H} m_{R}(\mathcal{M}, \mathcal{N}) \longrightarrow \operatorname{Hom}_{R}(\mathcal{M}(x), \mathcal{N}(x))$ induces an isomorphism

$$
\operatorname{Hom}_{\mathcal{R}}(\mathcal{M}, \mathcal{N}) \stackrel{\sim}{\longrightarrow} \operatorname{Hom}_{\mathcal{R}(x)}(\mathcal{M}(x), \mathcal{N}(x)) .
$$

A.1.9 Definition. - If $\mathcal{M}$, resp. $\mathcal{N}$ is a right, resp. left, "R-module", we define their "tensor product over $\mathcal{R}$ " as the $R$-module

$$
\mathcal{M} \otimes_{\mathcal{R}} \mathcal{N}:=\int^{\mathcal{R}} \mathcal{M} \otimes_{R} \mathcal{N}
$$

When $\mathcal{R}$ has a single object and is thus an ordinary ring, this is consistent with the usual tensor product over $\mathcal{R}$. Moreover, classical adjunction properties of tensor products extend to this context.

\section{A.1.10 Lemma. — There is a canonical isomorphism}

$$
\operatorname{Hom}_{R}\left(\mathcal{M} \otimes_{\mathcal{R}} \mathcal{N}, M\right) \stackrel{\sim}{\longrightarrow} \operatorname{Hom}_{\mathcal{R}}\left(\mathcal{N}, \operatorname{Hom}_{R}(\mathcal{M}, R)\right)
$$

natural in the left $\mathcal{R}$-module $\mathcal{N}$, the right $\mathcal{R}$-module $\mathcal{M}$, and the $R$-module $M$.

Proof. Since $\operatorname{Hom}_{R}(-, M)$ is left exact, we have

$$
\begin{aligned}
\operatorname{Hom}_{R}\left(\mathcal{M} \otimes_{\mathcal{R}} \mathcal{N}, M\right) & =\int_{R}\left(x^{\prime}, x\right) \mapsto \operatorname{Hom}_{R}\left(\mathcal{M}\left(x^{\prime}\right) \otimes_{R} \mathcal{N}(x), M\right) \\
& \stackrel{\sim}{\longrightarrow} \int_{R}\left(x^{\prime}, x\right) \mapsto \operatorname{Hom}_{R}\left(\mathcal{N}(x), \operatorname{Hom}_{R}\left(\mathcal{M}\left(x^{\prime}\right), M\right)\right) \\
& =\operatorname{Hom}_{\mathcal{R}}\left(\mathcal{N}, \operatorname{Hom}_{R}(\mathcal{M}, R)\right)
\end{aligned}
$$

where the isomorphism is classical adjunction over $R$. 
A.1.11 Corollary. - If $\mathcal{N}$ (resp. $\mathcal{M})$ is induced from the object $x$, the canonical map $\mathcal{M}(x) \otimes_{R} \mathcal{N}(x) \longrightarrow \int^{\mathcal{R}} \mathcal{M} \otimes_{R} \mathcal{N}$ induces an isomorphism

$$
\mathcal{M}(x) \otimes_{\mathcal{R}(x)} \mathcal{N}(x) \stackrel{\sim}{\longrightarrow} \mathcal{M} \otimes_{\mathcal{R}} \mathcal{N}
$$

Proof. For any $R$-module $M$, the previous lemma tells us that the functor $\operatorname{Hom}_{R}(-, M)$ takes the canonical map under consideration to the canonical map

$$
\int_{\mathcal{R}}\left(x^{\prime}, x\right) \mapsto \operatorname{Hom}_{\mathcal{R}}\left(\mathcal{N}(x), \operatorname{Hom}_{R}\left(\mathcal{M}\left(x^{\prime}\right), M\right)\right) \longrightarrow \operatorname{Hom}_{R}\left(\mathcal{N}(x), \operatorname{Hom}_{R}(\mathcal{M}(x), M)\right) .
$$

But as noted above, the latter map induces an isomorphism

$$
\operatorname{Hom}_{\mathcal{R}}\left(\mathcal{N}, \operatorname{Hom}_{R}(\mathcal{M}, M)\right) \stackrel{\sim}{\longrightarrow} \operatorname{Hom}_{\mathcal{R}(x)}\left(\mathcal{N}(x), \operatorname{Hom}_{R}(\mathcal{M}(x), M)\right),
$$

which means that the map under consideration induces an isomorphism

$$
\operatorname{Hom}_{R}\left(\mathcal{M} \otimes_{\mathcal{R}} \mathcal{N}, M\right) \stackrel{\sim}{\longrightarrow} \operatorname{Hom}_{R}\left(\mathcal{M}(x) \otimes_{\mathcal{R}(x)} \mathcal{N}(x), M\right) .
$$

\section{A.2 Morita equivalences}

A.2.1 "Bimodules" as functors between categories of "modules". - If $\mathcal{C}$ is an $R$-linear category with finite limits and colimits, one defines the end and coend of an $R$-bilinear functor $\mathcal{B}: \mathcal{R}^{\text {opp }} \times \mathcal{R} \longrightarrow \mathcal{C}$ by the same formulas as in A.1.7. These are objects of $\mathcal{C}$. An example of such a category $\mathcal{C}$ is $\mathcal{R}^{\prime}-$ Mod. Indeed, finite limits and colimits exist in this category and are computed terms by terms, e.g. $\left(\lim _{I} \mathcal{M}_{i}^{\prime}\right)\left(x^{\prime}\right)=\lim _{I} \mathcal{M}_{i}^{\prime}\left(x^{\prime}\right)$.

Now, let $\mathcal{B}$ be a " $\left(\mathcal{R}, \mathcal{R}^{\prime}\right)$-bimodule" as in A.1.6 and let $\mathcal{M}$ be an " $\mathcal{R}$-module". Then $\mathcal{H o m}_{R}(\mathcal{B}, \mathcal{M})$ is an $R$-trilinear functor $\mathcal{R}^{\text {opp }} \times \mathcal{R}^{\prime} \times \mathcal{R} \longrightarrow R$-Mod, and can be seen as an $R$ bilinear functor $\mathcal{H}: \mathcal{R}^{\text {opp }} \times \mathcal{R} \longrightarrow \mathcal{R}^{\prime}-$ Mod. The end $\int_{\mathcal{R}} \mathcal{H}$ of $\mathcal{H}$ is therefore a left " $\mathcal{R}^{\prime}$-module" that we will denote by $\operatorname{Hom}_{\mathcal{R}}(\mathcal{B}, \mathcal{M})$. In this way we obtain a functor

$$
\begin{aligned}
\mathcal{R}-\operatorname{Mod} & \rightarrow \mathcal{R}^{\prime}-\operatorname{Mod} \\
\mathcal{M} & \mapsto \operatorname{Hom}_{\mathcal{R}}(\mathcal{B}, \mathcal{M})
\end{aligned}
$$

Concretely, for any $x^{\prime} \in \mathcal{R}$, we have

$$
\operatorname{Hom}_{\mathcal{R}}(\mathcal{B}, \mathcal{M})\left(x^{\prime}\right)=\operatorname{Hom}_{\mathcal{R}}\left(\mathcal{B}\left(x^{\prime},-\right), \mathcal{M}\right)=\int_{\mathcal{R}} \operatorname{Hom}_{R}\left(\mathcal{B}\left(x^{\prime}, x\right), \mathcal{M}(x)\right) .
$$

On the other hand, let $\mathcal{M}^{\prime}$ be a left " $\mathcal{R}^{\prime}$-module". We get an $R$-trilinear functor $\mathcal{B} \otimes_{R} \mathcal{M}^{\prime}$ : $\mathcal{R} \times \mathcal{R}^{\prime \text { opp }} \times \mathcal{R}^{\prime} \longrightarrow R$ - Mod whence an $R$-bilinear functor $\mathcal{R}^{\prime \text { opp }} \times \mathcal{R}^{\prime} \longrightarrow \mathcal{R}-$ Mod. As above we get a functor

$$
\begin{aligned}
\mathcal{R}^{\prime}-\operatorname{Mod} & \rightarrow \mathcal{R}-\operatorname{Mod} \\
\mathcal{M}^{\prime} & \mapsto \mathcal{B} \otimes_{\mathcal{R}^{\prime}} \mathcal{M}^{\prime}
\end{aligned}
$$

with $\left(\mathcal{B} \otimes_{\mathcal{R}^{\prime}} \mathcal{M}^{\prime}\right)(x)=\mathcal{B}(-, x) \otimes_{\mathcal{R}^{\prime}} \mathcal{M}^{\prime}=\int^{\mathcal{R}^{\prime}} \mathcal{B}\left(x^{\prime}, x\right) \otimes_{R} \mathcal{M}^{\prime}\left(x^{\prime}\right)$. 
A.2.2 Proposition. - The functor $\mathcal{M}^{\prime} \mapsto \mathcal{B} \otimes_{\mathcal{R}^{\prime}} \mathcal{M}^{\prime}$ is left adjoint to the functor $\mathcal{M} \mapsto$ $\operatorname{Hom}_{\mathcal{R}}(\mathcal{B}, \mathcal{M})$.

Proof. We first define a counit. Let $\mathcal{M}$ be a left $\mathcal{R}$-module. For any $x \in \mathcal{R}$ and $x^{\prime} \in \mathcal{R}^{\prime}$, consider the canonical map

$$
\operatorname{Hom}_{\mathcal{R}}(\mathcal{B}, \mathcal{M})\left(x^{\prime}\right)=\operatorname{Hom}_{\mathcal{R}}\left(\mathcal{B}\left(x^{\prime},-\right), \mathcal{M}\right) \longrightarrow \operatorname{Hom}_{R}\left(\mathcal{B}\left(x^{\prime}, x\right), \mathcal{M}(x)\right)
$$

Taking the sum of the adjoint maps, we get a map

$$
\bigoplus_{x^{\prime} \in \mathcal{R}^{\prime}} \mathcal{B}\left(x^{\prime}, x\right) \otimes_{R} \operatorname{Hom}_{\mathcal{R}}(\mathcal{B}, \mathcal{M})\left(x^{\prime}\right) \longrightarrow \mathcal{M}(x) .
$$

It is tediously but easily checked that this map factors through the coend, giving a map

$$
\left(\mathcal{B} \otimes_{\mathcal{R}^{\prime}} \operatorname{Hom}_{\mathcal{R}}(\mathcal{B}, \mathcal{M})\right)(x)=\mathcal{B}(-, x) \otimes_{\mathcal{R}^{\prime}} \operatorname{Hom}_{\mathcal{R}}(\mathcal{B}, \mathcal{M}) \longrightarrow \mathcal{M}(x) .
$$

When varying $x$, this collection of maps defines a morphism of $\mathcal{R}$-modules

$$
\mathcal{B} \otimes_{\mathcal{R}^{\prime}} \operatorname{Hom}_{\mathcal{R}}(\mathcal{B}, \mathcal{M}) \longrightarrow \mathcal{M}
$$

which is clearly natural in $\mathcal{M}$.

Let us now define the unit. Let $\mathcal{M}^{\prime}$ be a left $\mathcal{R}^{\prime}$-module. For any $x \in \mathcal{R}$ and $x^{\prime} \in \mathcal{R}^{\prime}$, consider the canonical map

$$
\mathcal{B}\left(x^{\prime}, x\right) \otimes_{R} \mathcal{M}^{\prime}\left(x^{\prime}\right) \longrightarrow \mathcal{B}(-, x) \otimes_{\mathcal{R}^{\prime}} \mathcal{M}^{\prime}=\left(\mathcal{B} \otimes_{\mathcal{R}^{\prime}} \mathcal{M}^{\prime}\right)(x)
$$

Taking the product of the adjoint maps, we get a map

$$
\mathcal{M}^{\prime}\left(x^{\prime}\right) \longrightarrow \prod_{x \in \mathcal{R}} \operatorname{Hom}_{R}\left(\mathcal{B}\left(x^{\prime}, x\right),\left(\mathcal{B} \otimes_{\mathcal{R}^{\prime}} \mathcal{M}^{\prime}\right)(x)\right) .
$$

Another tedious and easy check insures that this map factors through the end, giving a map

$$
\mathcal{M}^{\prime}\left(x^{\prime}\right) \longrightarrow \operatorname{Hom}_{\mathcal{R}}\left(\mathcal{B}\left(x^{\prime},-\right), \mathcal{B} \otimes_{\mathcal{R}^{\prime}} \mathcal{M}^{\prime}\right)=\operatorname{Hom}_{\mathcal{R}}\left(\mathcal{B}, \mathcal{B} \otimes_{\mathcal{R}^{\prime}} \mathcal{M}^{\prime}\right)\left(x^{\prime}\right)
$$

Now, varying $x$, this collection of maps defines a morphism of $\mathcal{R}^{\prime}$-modules

$$
\mathcal{M}^{\prime} \longrightarrow \operatorname{Hom}_{\mathcal{R}}\left(\mathcal{B}, \mathcal{B} \otimes_{\mathcal{R}^{\prime}} \mathcal{M}^{\prime}\right)
$$

which is clearly natural in $\mathcal{M}^{\prime}$.

It remains to check that these maps satisfy the axioms of a counit-unit system. We leave the reader check in detail that this indeed follows from the classical adjunction between functors $\mathcal{B}\left(x^{\prime}, x\right) \otimes_{R}-$ and $\operatorname{Hom}_{R}\left(\mathcal{B}\left(x^{\prime}, x\right),-\right)$ for each pair of objects $x^{\prime}, x$.

A.2.5 Definition. - We say that $\mathcal{B}$ induces a Morita equivalence between $\mathcal{R}$ and $\mathcal{R}^{\prime}$ if the functors of the last proposition are equivalences of categories or, equivalently, if all maps (A.2.3) and (A.2.4) are isomorphisms.

The following result is relevant for our applications in the main body of this paper. 
A.2.6 Proposition. - Assume there is a map $\iota: \mathcal{R}^{\prime} \longrightarrow \mathcal{R}$ such that the following holds.

i) for all $x^{\prime} \in \mathcal{R}^{\prime}$, the left $\mathcal{R}$-module $x \mapsto \mathcal{B}\left(x^{\prime}, x\right)$ is induced from $\iota x^{\prime}$ and the right $\mathcal{R}^{\prime}$-module $y^{\prime} \mapsto \mathcal{B}\left(y^{\prime}, \iota x^{\prime}\right)$ is induced from $x^{\prime}$ in the sense of Definition A.1.4.

ii) for all $x^{\prime} \in \mathcal{R}^{\prime}$, the bimodule $\mathcal{B}\left(x^{\prime}, \iota x^{\prime}\right)$ induces a Morita equivalence between the $R$-algebras $\mathcal{R}\left(\iota x^{\prime}\right)$ and $\mathcal{R}^{\prime}\left(x^{\prime}\right)$.

iii) any non zero object $x \in \mathcal{R}$ is isomorphic to some $\iota x^{\prime}$ in $\mathcal{R}$.

Then $\mathcal{B}$ induces a Morita equivalence between $\mathcal{R}$ and $\mathcal{R}^{\prime}$. Moreover, the two associated equivalences of categories preserve the propery of being cartesian.

Proof. We first show that the map (A.2.3) is an isomorphism for any $\mathcal{R}^{\prime}$-module $\mathcal{M}^{\prime}$. To this end we shall evaluate it at any $\iota x^{\prime}$, for $x^{\prime} \in \mathcal{R}^{\prime}$. Our assumption i) provides us with an isomorphism

$$
\operatorname{Hom}_{\mathcal{R}}(\mathcal{B}, \mathcal{M})\left(x^{\prime}\right) \stackrel{\sim}{\longrightarrow} \operatorname{Hom}_{\mathcal{R}\left(\iota x^{\prime}\right)}\left(\mathcal{B}\left(x^{\prime}, \iota x^{\prime}\right), \mathcal{M}\left(\iota x^{\prime}\right)\right)
$$

as in example A.1.8, as well as an isomorphism

$$
\mathcal{B}\left(x^{\prime}, \iota x^{\prime}\right) \otimes_{\mathcal{R}^{\prime}\left(x^{\prime}\right)} \operatorname{Hom}_{\mathcal{R}}(\mathcal{B}, \mathcal{M})\left(x^{\prime}\right) \stackrel{\sim}{\longrightarrow}\left(\mathcal{B} \otimes_{\mathcal{R}^{\prime}} \operatorname{Hom}_{\mathcal{R}}(\mathcal{B}, \mathcal{M})\right)\left(\iota x^{\prime}\right),
$$

thanks to Lemma A.1.11. Combining these two isomorphisms gives the following one

$$
\left(\mathcal{B} \otimes_{\mathcal{R}^{\prime}} \operatorname{Hom}_{\mathcal{R}}(\mathcal{B}, \mathcal{M})\right)\left(\iota x^{\prime}\right) \simeq \mathcal{B}\left(x^{\prime}, \iota x^{\prime}\right) \otimes_{\mathcal{R}^{\prime}\left(x^{\prime}\right)} \operatorname{Hom}_{\mathcal{R}\left(\iota x^{\prime}\right)}\left(\mathcal{B}\left(x^{\prime}, \iota x^{\prime}\right), \mathcal{M}\left(\iota x^{\prime}\right)\right)
$$

Tracking backward definitions shows that this isomorphism identifies the counit (A.2.3) at $\iota x^{\prime}$ with the evaluation map of the RHS. But our hypothesis ii) implies that this evaluation map is an isomorphism. Hence we have proved that the counit (A.2.3) is an isomorphism at any object of the form $\iota x^{\prime}$. By hypothesis iii), it is an isomorphism at any $x \in \mathcal{R}$.

The proof that the map (A.2.4) is an isomorphism is very similar, except that we don't need hypothesis ii) in this case.

It remains to prove the last assertion about cartesian modules. Let $\mathcal{M}$ be an $\mathcal{R}$-module. It suffices to prove that $\mathcal{M}$ is cartesian if and only if $\mathcal{M}^{\prime}=\operatorname{Hom}_{\mathcal{R}}(\mathcal{B}, \mathcal{M})$ is cartesian. To this aim, we have to study the map $a_{y^{\prime}, x^{\prime}}^{*}$ of A.1.4 for the $\mathcal{R}^{\prime}$-module $\mathcal{M}^{\prime}=\operatorname{Hom}_{\mathcal{R}}(\mathcal{B}, \mathcal{M})$ and any $x^{\prime}, y^{\prime} \in \mathcal{R}^{\prime}$. We leave the reader convince himself that this map is the following composition of maps.

$$
\begin{aligned}
a_{y^{\prime}, x^{\prime}}^{*}: \operatorname{Hom}_{\mathcal{R}}(\mathcal{B}, \mathcal{M})\left(y^{\prime}\right) & \stackrel{\sim}{\longrightarrow} \operatorname{Hom}_{\mathcal{R}\left(\iota y^{\prime}\right)}\left(\mathcal{B}\left(y^{\prime}, \iota y^{\prime}\right), \mathcal{M}\left(\iota y^{\prime}\right)\right) \\
& \stackrel{a_{\iota y^{\prime} \iota x^{\prime}}^{*}}{\longrightarrow} \operatorname{Hom}_{\mathcal{R}\left(\iota y^{\prime}\right)}\left(\mathcal{B}\left(y^{\prime}, \iota y^{\prime}\right), \operatorname{Hom}_{\mathcal{R}\left(\iota x^{\prime}\right)}\left(\mathcal{R}\left(\iota y^{\prime}, \iota x^{\prime}\right), \mathcal{M}\left(\iota x^{\prime}\right)\right)\right) \\
& \stackrel{\sim}{\longrightarrow} \operatorname{Hom}_{\mathcal{R}\left(\iota x^{\prime}\right)}\left(\mathcal{B}\left(y^{\prime}, \iota y^{\prime}\right) \otimes_{\mathcal{R}\left(\iota y^{\prime}\right)} \mathcal{R}\left(\iota y^{\prime}, \iota x^{\prime}\right), \mathcal{M}\left(\iota x^{\prime}\right)\right) \\
& \stackrel{\sim}{\longrightarrow} \operatorname{Hom}_{\mathcal{R}\left(\iota x^{\prime}\right)}\left(\mathcal{B}\left(y^{\prime}, \iota x^{\prime}\right), \mathcal{M}\left(\iota x^{\prime}\right)\right) \\
& \stackrel{\sim}{\longrightarrow} \operatorname{Hom}_{\mathcal{R}\left(\iota x^{\prime}\right)}\left(\mathcal{R}^{\prime}\left(y^{\prime}, x^{\prime}\right) \otimes_{\mathcal{R}^{\prime}\left(x^{\prime}\right)} \mathcal{B}\left(x^{\prime}, \iota x^{\prime}\right), \mathcal{M}\left(\iota x^{\prime}\right)\right) \\
& \stackrel{\sim}{\longrightarrow} \operatorname{Hom}_{\mathcal{R}^{\prime}\left(x^{\prime}\right)}\left(\mathcal{R}^{\prime}\left(y^{\prime}, x^{\prime}\right), \operatorname{Hom}_{\mathcal{R}\left(\iota x^{\prime}\right)}\left(\mathcal{B}\left(x^{\prime}, \iota x^{\prime}\right), \mathcal{M}\left(\iota x^{\prime}\right)\right)\right) \\
& \stackrel{\sim}{\longrightarrow} \operatorname{Hom}_{\mathcal{R}^{\prime}\left(x^{\prime}\right)}\left(\mathcal{R}^{\prime}\left(y^{\prime}, x^{\prime}\right), \operatorname{Hom}_{\mathcal{R}}(\mathcal{B}, \mathcal{M})\left(x^{\prime}\right)\right)
\end{aligned}
$$


In the first and last lines, we have applied A.1.8, using that $y \mapsto \mathcal{B}\left(y^{\prime}, y\right)$ is induced from $\iota y^{\prime}$. The third and sixth lines are classical adjunction properties. The fourth line again uses that $y \mapsto \mathcal{B}\left(y^{\prime}, y\right)$ is induced from $\iota y^{\prime}$. The fifth line is given by the right $\mathcal{R}^{\prime}$-module structure on $\mathcal{B}\left(-, \iota x^{\prime}\right)$. It is an isomorphism, by our hypothesis that $y^{\prime} \mapsto \mathcal{B}\left(y^{\prime}, \iota x^{\prime}\right)$ is induced from $x^{\prime}$. It follows from this sequence of maps that

$$
a_{\iota y^{\prime}, \iota x^{\prime}}^{*} \text { is an isomorphism if and only if } a_{y^{\prime}, x^{\prime}}^{*} \text { is an isomorphism. }
$$

Going back to the definition of cartesian modules, we see that, thanks to hypothesis iii), what remains to prove is

$$
\mathcal{R}^{\prime}\left(y^{\prime}, x^{\prime}\right) \neq 0 \Leftrightarrow \mathcal{R}\left(\iota y^{\prime}, \iota x^{\prime}\right) \neq 0 .
$$

But by hypothesis i) we have the folloving isomorphisms

$$
\mathcal{R}^{\prime}\left(y^{\prime}, x^{\prime}\right) \otimes_{\mathcal{R}^{\prime}\left(x^{\prime}\right)} \mathcal{B}\left(x^{\prime}, \iota x^{\prime}\right) \stackrel{\sim}{\longrightarrow} \mathcal{B}\left(y^{\prime}, \iota x^{\prime}\right) \stackrel{\sim}{\sim} \mathcal{B}\left(y^{\prime}, \iota y^{\prime}\right) \otimes_{\mathcal{R}\left(\iota y^{\prime}\right)} \mathcal{R}\left(\iota y^{\prime}, \iota x^{\prime}\right)
$$

and by hypothesis ii), the functors $-\otimes_{\mathcal{R}^{\prime}\left(x^{\prime}\right)} \mathcal{B}\left(x^{\prime}, \iota x^{\prime}\right)$ and $\mathcal{B}\left(y^{\prime}, \iota y^{\prime}\right) \otimes_{\mathcal{R}\left(\iota y^{\prime}\right)}-$ are faithful.

\section{References}

[1] C. Bonnafé, J.-F. Dat, and R. Rouquier. Derived categories and Deligne-Lusztig varieties II. arXiv:1511.04714v2 [math.RT], 2015.

[2] C. Bonnafé and R. Rouquier. Catégories dérivées et variétés de Deligne-Lusztig. Publ. Math. Inst. Hautes Études Sci., (97):1-59, 2003.

[3] M. Cabanes and M. Enguehard. Representation theory of finite reductive groups, volume 1 of New Mathematical Monographs. Cambridge University Press, Cambridge, 2004.

[4] J.-F. Dat. Dependence of the Deligne-Lusztig induction on the parabolic subgroup. http://webusers.imj-prg.fr/ jean-francois.dat/recherche/travaux.php, 2014.

[5] J.-F. Dat. A functoriality principle for blocks of linear $p$-adic groups. http://webusers.imj-prg.fr/ jean-francois.dat/recherche/travaux.php, 2016.

[6] D. Helm. The Bernstein center of the category of smooth $W(k)\left[G L_{n}(F)\right]$-modules. http://wwwf.imperial.ac.uk/ dhelm/, 2015.

[7] G. Lusztig. Green functions and character sheaves. Ann. of Math. (2), 131(2):355-408, 1990.

[8] R. Meyer and M. Solleveld. Resolutions for representations of reductive $p$-adic groups via their buildings. J. Reine Angew. Math., 647:115-150, 2010.

[9] M.-F. Vignéras. Induced $R$-representations of $p$-adic reductive groups. Selecta Math. (N.S.), 4(4):549-623, 1998.

[10] H Wang. L'espace symétrique de Drinfeld et correspondance de Langlands locale II. arXiv:1402.1965 [math.NT], 2014.

Jean-François Dat. Institut de Mathématiques de Jussieu-PRG, Université Pierre et Marie Curie, 4 place Jussieu, 75005 Paris, France. e-mail : jean-francois.dat@imj-prg.fr 